\title{
A SKELETON KEY TO ABHYANKAR'S PROOF OF EMBEDDED RESOLUTION OF CHARACTERISTIC P SURFACES*
}

\author{
STEVEN DALE CUTKOSKY ${ }^{\dagger}$
}

Dedicated to Professor Hironaka, on the occasion of his 80th birthday

Key words. Singularity, resolution, surface, positive characteristic, valuation.

AMS subject classifications. 14B05, 14E14, 13A18.

This paper analyzes and simplifies Abhyankar's proof of embedded resolution of surface singularities in positive characteristic. Abhyankar's proof is obtained by combining the results in the papers [2], [4], [5], [6] and the first two chapters of the book [7]. This proof is extremely influential, but because of its length and complexity, is not generally well known and understood. In this article, I have written a report on the proof, hoping to make the main ideas more generally known. I give complete proofs of the essential parts of the proof. Some lemmas, which are given complete self contained proofs in Abhyankar's work, are merely stated and cited in this paper. Some of these cited results can be proven directly without great difficulty. I have made substantial simplifications in the original proofs, but have made a point of not making simplications which eliminate an original and interesting idea which could possibly have application to resolution in higher dimension.

Resolution of singularities in characteristic zero and in all dimensions was first proven by Hironaka [26]. More recently, there have been significant simplifications of this proof, including in [9], [11], [12], [20], [21], [28], [29], [34], [44], [45]. The first proof of resolution of surface singularities in characteristic $p>0$ was by Abhyankar [1]. There have been other proofs of resolution of surface singularities in characteristic $p>0$ since this time, including the proof analyzed in this paper, and proofs by Hironaka [27], Lipman [37], Hauser [24] and Cossart, Jannsen and Saito [16]. The first proof of resolution of singularities of 3 -folds in positive characteristic $p>5$ was given by Abhyankar [7], using the embedded resolution theorems for surfaces analyzed in this paper. A greatly simplified proof appears in [18], using Hironaka's algorithm [27] for embedded resolution of surface singularities. Recently, Cossart and Piltant have proven resolution of singularities of 3 -folds in all characteristics [14], [15]. Some of the recent papers attacking resolution in higher dimensions and positive characteristic are [13], [19], [30], [31], [32], [33], [35], [39], [42], and [43].

Abhyankar's proof of embedded resolution of surface singularities is essentially a generalization of Zariski's characteristic zero proof [46] of embedded resolution of surface singularities. Zariski's final global proof, deducing the Theorem of Beppo Levi from local uniformization, extends without much difficulty to characteristic $p>0$. The essential point where Zariski's proof does not extend to characteristic $p>0$ is in local uniformization of a particular type of valuation $\nu$ which dominates a normal local domain of dimension two. This difficult case occurs when $\nu$ is rational nondiscrete. For the most part, for simplicity, we restrict to the analysis of this fundamental case.

The global argument used to deduce global resolution from local uniformization does not extend to dimension three, even in characteristic zero, as birational geometry

\footnotetext{
*Received April 3, 2010; accepted for publication May 20, 2011.

†202 Mathematical Sciences Bldg, University of Missouri, Columbia, MO 65211, USA (cutkoskys@ missouri.edu). Partially supported by NSF.
} 
is tremendously more complicated in higher dimensions. Even the algorithm of Beppo Levi fails in dimension three [41].

Abhyankar's proof of local uniformization is by consideration of a sequence of blow ups of points along the valuation, until a good form is obtained, from which it is easy to make a reduction in multiplicity by blowing up a sequence of nonsingular curves. This is the philosophy of the good point algorithm which Abhyankar considers in a later paper [8]. In characteristic zero, this method gives a very simple and elegant global proof of resolution of surface singularities [40], [17]. It is not so difficult to prove from local uniformization, stated in Theorem 4.1, that the good point algorithm yields a global proof of resolution of singularities for characteristic $p>0$ surfaces.

Ramification theoretic methods and embedded resolution of ideals in regular local rings of dimension two are used to reduce the problem of reduction of the order of an element $f$ in a regular local ring of dimension three (along a valuation) to the situation where $f$ is a monic polynomial of degree $p^{n}$ of multiplicity $p^{n}$. The proof of this result may extend to a local result along a valuation in higher dimensions, with the assumption that embedded resolution is true in codimension 1. Recently, strong global versions of this result in all dimensions have been found by Hironaka [31], and in the work of Benito and Villamayor [10]. A reduction to the inseparable case has been found in all dimensions, locally along a valuation, by Temkin [43].

The most striking part of Abhyankar's proof is the argument for reduction of order of a monic polynomial of order $p^{n}$. The first interesting point is that the problem is set up as an inductive statement. It is phrased as a problem on reduction of order of a polynomial $f(Z)$, with coefficients in a regular local ring of dimension two. By performing only blow ups of the two dimensional regular local ring a stable form of the polynomial is obtained, which is adequate to prove reduction of order of a local equation of a surface in a 3 -fold. Another interesting point is a reduction to the case where $f(Z)$ is almost purely inseparable; that is $f(Z)$ transforms as if it had a form $f(Z)=Z^{p^{n}}+F$ with $F \in R$. To obtain this reduction, resolution of the ArtinSchreier case must be completely solved. Then ramification theoretic methods are used in an ingenious way. Reduction for the Artin-Schreier case is accomplished in Section 4 of this paper. Recently, Cossart and Piltant [15] have proven resolution of the Artin-Schreier case in dimension three. The proof is extremely long and complex.

Abhyankar's algorithm of reduction for a polynomial $f(Z)$ of degree $p^{n}$ is by studying how the Newton Polygon of $f(0)$ (which is a polynomial or series in two variables) changes under translations or "cleaning" (replacing $Z$ with $Z+r$ for some $r \in R$ ), and under quadratic transforms of $R$. The singularity of $f(Z)$ is tracked by considering an $R$-type $(a, b, c)$ or an $R$-antitype $(b, a, d)$. The number $c$ can in fact go up after blowing up and cleaning. This corresponds to the concept of "shade" in [25]. The subtle bracket $[b, c]$ (defined after Lemma 7.3 ) is used to control $c$ under blowing up.

This part of the argument (Chapter 7) may appear at first to be a web of overwhelming complexity. However, many deep ideas are incorporated into the proof. The proof demonstrates in a very clear way some of the problems which arise in resolution in higher dimension, and realizes in the "simplest" case the natural algebraic approach to resolution in positive characteristic.

The numbers $a, b, d$ are related to the invariant $\left(\beta, \frac{1}{e}, \alpha\right)$ which is the main resolution invariant in Hironaka's resolution algorithm [27], [24], [18], [16]. Hironaka considers a Newton polygon which is a projection of the coefficients of all the terms of the polynomial. His resolution algorithm (for dimension two) is to apply the resolu- 
tion algorithm of Beppo Levi directly, and show that this invariant always drops under resolution. Hironaka's invariant can however go up under the good point algorithm for resolution.

\section{An outline of the proof.}

Reduction of global resolution of singularities to local uniformization. Suppose that $Y$ is a surface contained in a nonsingular 3-fold $X$, over an algebraically closed field $\bar{k}$ of characteristic $p>0$.

It is proven in Theorem 9.12 and Corollary 9.13 that there exists a finite sequence of blow ups of points and nonsingular curves

$$
X_{n} \rightarrow X_{n-1} \rightarrow \cdots \rightarrow X_{1} \rightarrow X
$$

such that the center blown up by each $\Phi_{i}: X_{i} \rightarrow X_{i-1}$ is in the locus where the strict transform $Y_{i}$ of $Y$ on $X_{i}$ is singular, and the strict transform of $Y_{n}$ on $X_{n}$ is nonsingular.

Let $e$ be the largest multiplicity of a point on $Y$. A permissible blow up of $X$ is the blow up of a point or nonsingular curve contained in the (closed) locus $\operatorname{Sing}_{e}(Y)$ of points of multiplicity $e$ on $Y$. The blow up is strictly permissible if the center blown up is a point or nonsingular curve contained in $\operatorname{Sing}_{e}(Y)$. Under a permissible blow up, the multiplicity of the strict transform cannot go up. Further, while there is in general not a "hypersurface of maximal contact", there is an approximation to one which has some good properties. Since a point of $Y$ is nonsingular if and only if it has multiplicity 1 , by induction on $e$, we are reduced to constructing a sequence (1) of permissible blow ups such that all points on the strict transform $Y_{n}$ of $Y$ are of multiplicity less than $e$.

We will say that $Y$ is prepared if all irreducible curves in $\operatorname{Sing}_{e}(Y)$ are nonsingular, at most two curves in $\operatorname{Sing}_{e}(Y)$ pass through any given point of $\operatorname{Sing}_{e}(Y)$, and these two curves intersect transversally at $p$ if this happens.

After a few permissible blow ups (Theorem 9.4), we obtain the situation that $Y_{1}$ is prepared, where $Y_{1}$ is the strict transform of $Y$. If $Y_{2}$ is the strict transform of $Y_{1}$ after a further sequence of permissible blowups, we have that $Y_{2}$ is also prepared. We may assume that $Y$ is prepared.

The main resolution theorem, Theorem 9.12, is that any sequence of strictly permissible blowups will eventually terminate in an $X_{n}$ such that $Y_{n}$ contains no points of multiplicity $e$. This is the "Theorem of Beppo Levi". The major ingredient in the proof is local uniformization: for every 0-dimensional valuation $\nu$ of the function field $\bar{k}(X)$ there exists a finite sequence of permissible blow ups $\bar{X}_{m} \rightarrow X$ such that the center of $\nu$ on the strict transform $\bar{Y}_{m}$ of $Y$ is less than $e$ at the center of $\nu$. The proof of local uniformization will be discussed in the next subsection. The obstruction to constructing a global resolution is that the choice of centers in this local resolution depends on the valuation $\nu$, so different resolutions for different valuations may not patch globally. A valuation gives a convenient way to interpret birational geometry locally; by the valuative criterion of properness, for any projective variety $Z$ with function field $K$, and valuation $\nu$ of $K$, there exists a unique local $\operatorname{ring} \mathcal{O}_{Z, q}$ of $Z$ such that the valuation ring of $\nu$ dominates $\mathcal{O}_{Z, q} . q$ is called the center of $\nu$ on $Z$. If the residue field of $\nu$ is equal to the algebraically closed field $\bar{k}$, then $\nu$ is called 0-dimensional. The center of a 0-dimensional valuation is always a closed point.

Assuming local uniformization, we now indicate how we deduce termination of a sequence of strictly permissible blow ups. Assume that we can construct such a 
sequence which does not terminate, so that we have a sequence of infinite length

$$
\cdots \rightarrow X_{i} \stackrel{\Phi_{i}}{\rightarrow} X_{i-1} \rightarrow \cdots \rightarrow X_{1} \stackrel{\Phi_{1}}{\rightarrow} X_{0}=X
$$

of strictly permissible blow ups. Since all of these blow ups are strictly permissible, we can find an infinite sequence of points $p_{i} \in X_{i}$ such that $\Phi_{i}\left(p_{i}\right)=p_{i-1}$ for all $i, p_{i}$ has multiplicity $e$ on the strict transform $Y_{i}$ of $Y$ on $X_{i}$, and that infinitely many of the $p_{i}$ are on the center blown up by $\Phi_{i+1}$. We can then assume, without loss of generality, that each $p_{i}$ is on the center blown up by $\Phi_{i+1}$. There exists a 0 -dimensional valuation $\nu$ of $\bar{k}(X)$ whose center on $X_{i}$ is $p_{i}$ for all $i$ in the sequence (2).

We now consider a finite sequence of permissible blow ups

$$
\bar{X}_{m} \rightarrow \cdots \rightarrow \bar{X}_{1} \rightarrow X
$$

such that the strict transform $\bar{Y}_{m}$ of $Y$ on $\bar{X}_{m}$ has multiplicity less than $e$ at the center of $\nu$. Such a sequence exists by local uniformization (Theorem 9.7).

We compare the sequences (2) and (3) to modify the sequence (2) by essentially splicing in (3), to obtain the impossible conclusion that the strict transform of $Y$ on $\bar{X}_{m}$ has multiplicity $e$ at the center of $\nu$. The fact that infinitely many of the $\Phi_{i}$ must in fact be the blow up of the point $p_{i-1}$ is essential in this argument. This contradiction shows that the sequence (2) must in fact be finite.

Our proof of Theorem 9.12 extends without difficulty to the case where $Y$ is a reduced, but not necessarily integral, surface. The analysis in Sections $5-8$ and 11 of [18] reduces the proof of the fundamental theorems on embredded resolution of a surface in a nonsingular 3-fold (stated in Theorem 9.14) and principalization of ideal sheaves on a nonsingular 3-fold (stated in Theorem 9.15) to the Theorem of Beppo Levi for reduced surfaces. This part of the proof involves no essential differences between characteristic zero and characteristic $p>0$. in Sections 9 and 10 of [18], we use Hironaka's resolution algorithm to prove the Theorem of Beppo Levi for reduced surfaces.

Local uniformization. Suppose that $\nu$ is a 0 -dimensional valuation of the function field $\bar{k}(X)$ of $X$. The goal (realized in Theorem 9.7 and Corollary 9.8) is to construct a sequence of blow ups of points which are the center of $\nu$,

$$
X_{n} \rightarrow X_{n-1} \rightarrow \cdots \rightarrow X_{1} \rightarrow X
$$

until we obtain a good form for a local equation of the strict transform $Y_{n}$ of $Y$ on $X_{n}$, from which we may deduce that after a further sequence of permissible blow ups of curves we obtain a reduction in multiplicity at the center of $\nu$.

By a theory of the tangent cone (Lemma 9.1) in a sequence (4), we have regular parameters $x_{i}, y_{i}, z_{i}$ in the local ring $R_{i}$ of the center of $p_{i}$ on $X_{i}$ which are related by

$$
x_{i}=x_{i+1}, y_{i}=x_{i+1}\left(y_{i+1}+\alpha_{i+1}\right), z_{i}=x_{i+1}\left(z_{i+1}+\lambda_{i+1}\right) \text { with } \alpha_{i+1} \in \bar{k}, \lambda_{i+1} \in \bar{k}\left[x_{i}, y_{i}\right],
$$

or

$$
x_{i}=x_{i+1} y_{i+1}, y_{i}=y_{i+1}, z_{i}=y_{i+1}\left(z_{i+1}+\lambda_{i+1}\right) \text { with } \lambda_{i+1} \in \bar{k}\left[x_{i}, y_{i}\right] .
$$

Let $S_{0}=\bar{k}[[x, y]]$ and $S_{i}=S_{0}\left[x_{i}, y_{i}\right]_{\left(x_{i}, y_{i}\right)}$ for $i \geq 1$, which are regular local rings of dimension two.

The valuation $\nu$ of $\bar{k}(X)$ induces a 0 -dimensional valuation of the quotient field $K$ of $S$, which we will also call $\nu$. The infinite sequence of quadratic transforms

$$
S_{0} \rightarrow S_{1} \rightarrow \cdots \rightarrow S_{n} \rightarrow \cdots
$$


is dominated by $\nu$.

The desired good form which we seek is an expression for a (formal) local equation $w=0$ of $Y_{n}$ at $p_{n}$ of the form

$$
w=\left(z_{n}\right)^{e}+\sum_{j=1}^{e} g_{j} x_{n}^{a j} y_{n}^{b j}\left(z_{n}\right)^{e-j}
$$

with $g_{e} \in M\left(\hat{S}_{n}\right)$ (the maximal ideal of $S_{n}$ ), and $\operatorname{ord}_{\hat{S}_{n}} g_{j^{\prime}}<j^{\prime}$ for some $j^{\prime}$ with $1 \leq j^{\prime} \leq e$.

To show that we can obtain such a good form, we solve a slightly different problem. We start with a formal local equation $f(z)=0$ of $Y$ at $p$ with

$$
f(z)=z^{e}+a_{1} z^{e-1}+\cdots+a_{e},
$$

for some $a_{i} \in S$.

We then consider the monic polynomial $f(Z)$ in the polynomial $\operatorname{ring} S[Z]$ over $S$. We define the order $\operatorname{ord}_{S} f(Z)$ to be $\min \left\{\operatorname{ord}_{S}\left(a_{i}\right)+(e-i)\right\}$. Now by Theorem 8.1, there exists a number $n, r \in S_{n}$, and $u, v \in \mathbb{N}$ such that if we make the substitution $g(Z)=\left(x_{n}^{u} y_{n}^{v}\right)^{-e} f\left(x_{n}^{u} y_{n}^{v} Z+r\right)$, then we have $g(Z) \in \hat{S}_{n}[Z]$ and $0<\operatorname{ord}_{\hat{S}_{n}} g(Z)<e$. Finally, we compare this expression with our regular parameters $x_{n}, y_{n}, z_{n}$ in $R_{n}$, using Lemma 9.6, to obtain a local equation $w=0$ of $Y_{n}$ in $X_{n}$ which has the desired good form.

We will now discuss the proof of Theorem 8.1 in the essential case when the valuation $\nu$ (of the quotient field of $S$ ) is rational nondiscrete. This is by far the hardest case, and is the situation where differences between characteristic zero and $p$ are most evident. The remaining cases of valuations are essentially "toric" (The irrational case is for instance handled in Section 1 of [1]). The rational nondiscrete condition means that the sequence of blow ups (5) is as complicated as possible (a precise description is given in Lemma 2.2). We will write $(a, b) \equiv 0(m)$ if both integers $a$ and $b$ are divisible by $m$.

By Lemmas 8.4 and 8.5, it suffices to show that one of the following three conditions hold:

$$
\text { There exists } s \in S \text { such that } f(Z+s)=Z^{n} \text { or }
$$

There exists a number $k$ and $s \in S_{k}$ such that

$$
f(Z+s)=Z^{e}+x_{k}^{t_{1}} g_{1} Z^{e-1}+\cdots+x_{k}^{t_{e-1}} g_{e-1} Z+x_{k}^{t_{e}} g_{e}
$$

where $g_{i} \in S_{k}$ are not divisible by $x_{k}$, and there exists an integer $u$ with $0<u<e$ such that $g_{u}$ is a unit in $S_{k}, t_{j} \geq \frac{j}{u} t_{u}$ for $1 \leq j \leq u$ and $t_{j}>\frac{j}{u} t_{u}$ for $u<j \leq e$, or

There exists a number $k$ and $s \in S_{k}$ such that

$$
f(Z+s)=Z^{e}+x_{k}^{t_{1}} g_{1} Z^{e-1}+\cdots+x_{k}^{t_{e-1}} g_{e-1} Z+x_{k}^{t_{e}} g_{e}
$$

(8) where $g_{i} \in S_{k}$ are not divisible by $x_{k}$ and $t_{i} \geq \frac{i}{e} t_{e}$ for $1 \leq i \leq e$. Further, $\operatorname{ord}_{\left(R_{k}\right)_{x_{k} R_{k}}}\left(g_{e}\right)=c$ with $\left(t_{e}, c\right) \not \equiv 0(e)$ and $c=0$ if the degree of every nonconstant monic irreducible factor of $f(Z)$ in $K[Z]$ is not divisible by $p$, and $c \leq \frac{e}{p}$ if the degree of some nonconstant monic irreducible factor of $f(Z)$ is divisible by $p$. 
Using ramification theory, and the fact that appropriate discriminants (in a regular local ring of dimension two) can be made to be simple normal crossing divisors after enough blow ups, we reduce to the case where $f(Z) \in K[Z]$ is irreducible and $\nu$ does not split in $K[Z] /(f(Z))$. Now using Abhyankar's extension (Theorem 8.2) to positive characteristic of Jung's theorem on ramification over a SNC divisor in characteristic zero, we reduce to the case where $f(Z)$ is irreducible in $K[Z]$ of degree $m=p^{n}$, and $\nu$ does not split in $K[Z] /(f(Z))$. This final, and most difficult, case is discussed in the next subsection.

Reduction for monic polynomials of degree $\mathbf{m}=\mathbf{p}^{\mathbf{n}}$. In this subsection, we assume that $R$ is a regular local ring of dimension two, containing an algebraically closed field $\bar{k}$ which is isomorphic to its residue field. Suppose that $\nu$ is a valuation of the quotient field $K$ of $R$ which dominates $R$. Suppose that $L$ is a field extension of $K$, and $z \in L$ is not in $K$ and is integral over $R$. Suppose that $m=p^{n}=[K(z): K]$ and $f(Z) \in K[Z]$ is the minimal polynomial of $z$ over $K$.

The coefficients of $f(Z)$ are in $R$ since $z$ is integral over $R$. Write

$$
f(Z)=Z^{m}+f_{1} Z^{m-1}+\cdots+f_{m-1} Z+F
$$

with $f_{1}, \ldots, f_{m-1}, F \in R$.

The objective (realized in Theorem 7.8) is to find an index $k$ in the sequence of quadratic transforms

$$
R=R_{0} \rightarrow R_{1} \rightarrow \cdots
$$

along $\nu$ such that:

There exists a number $k$ and $r \in R_{k}$ such that

$$
f(Z+r)=Z^{m}+x_{k}^{t_{1}} g_{1} Z^{m-1}+\cdots+x_{k}^{t_{m-1}} g_{m-1} Z+x_{k}^{t_{m}} g_{m}
$$

where $g_{i} \in R_{k}$ are not divisible by $x_{k}$ and $t_{i} \geq \frac{i}{m} t_{m}$ for $1 \leq i \leq m$. Further, $\operatorname{ord}_{\left(R_{k}\right)_{x_{k} R_{k}}}\left(g_{m}\right)=c$ with $c<\frac{m}{p}$ and $\left(t_{m}, c\right) \not \equiv 0(m)$.

The assumptions that $\nu$ does not split in $K(z)$ and $f(Z)$ is irreducible in $K[Z]$ are necessary for obtaining this form. The method of proof is to perform two types of operations:

1. Perform a quadratic transform $R_{i} \rightarrow R_{i+1}$ along $\nu$.

2. Make an $R_{i}$-translate; that is replace $f(Z)$ with $f_{i}(Z)=f\left(Z+r_{i}\right)$ for some $r_{i} \in R_{i}$.

Let

$$
f(Z)=Z^{m}+f_{1} Z^{m-1}+\cdots+f_{m-1} Z+F
$$

with $f_{1}, \ldots, f_{m-1}, F \in R$. The analysis of the effect of these operations on $f(Z)$ is made especially complicated by the presence of the terms $f_{i}$ for $1 \leq i \leq m-1$. It turns out that for $i$ sufficently large in the quadratic sequence (9) these terms do not interfere too much with the term $F$, so that we can almost assume that these terms are not there at all. This good state of affairs occurs when $f(Z)$ is $R_{i}$-permissible. It means that $f(Z)$ is of nonsplitting type relative to $\operatorname{ord}_{R_{i}}$; that is

$$
\operatorname{ord}_{R_{i}} f_{q} \geq \frac{q}{m} \operatorname{ord}_{R_{i}} F
$$


for $0<q<m$, and $f(Z)$ is of ramified type relative to $\operatorname{ord}_{\left(R_{i}\right)_{x_{i} R_{i}}}$; that is if $f_{q}=x_{i}^{a_{q}} g_{q}$ with $g_{q} \in R$ and $x_{i} \not g_{q}$ for $0<q<m$ and $F=x_{i}^{a_{m}} G$ with $G \in R_{i}$ and $x_{i} \chi \chi G$, then

$$
a_{q}>\frac{q}{m} a_{m}
$$

for $0<q<m$. Further, these properties continue to hold after making an $R_{i^{-}}$ translate.

An essential result that we will return to a little later, is to show that $f(Z)$ is $R_{i}$-permissible for all large enough $i$. This is proven in Theorem 7.2. Assuming this result, we can then assume, without loss of generality, that $f(Z)$ is $R_{i}$-permissible for all $i$.

An intricate analysis is made, in Section 7, of how the constant term $F=f(0)$ of $f(Z)$ changes under quadratic transforms, while making suitable $R_{i}$-translates. This can be considered as a generalization of resolution of plane curve singularities, with the twist that monomials which are $p^{n}$-th powers are removed. After each quadratic transform, we take an $R_{i}$-translate $f_{i}(Z)$ of $f(Z)$.

To measure how much progress has been made towards reaching a form of the type of (10), there are two types of intermediate forms which are recorded: $f_{i}(Z)$ of $R_{i}$-type $\left(a_{i}, b_{i}, c_{i}\right)$ and of $R_{i}$-antitype $\left(b_{i}, a_{i}, d_{i}\right)$. In each of these types we require that $x_{i}^{a_{i}} y_{i}^{b_{i}}$ divides the "constant term" $f_{i}(0)$. In $\left(a_{i}, b_{i}, c_{i}\right)$-type we have that $f_{i}(0)$ has a nonzero $x_{i}^{a_{i}} y_{i}^{b_{i}+c_{i}}$ term with $\left(a_{i}, b_{i}+c_{i}\right) \not \equiv 0(m)$. In $\left(b_{i}, a_{i}, d_{i}\right)$-antitype we have that $f_{i}(0)$ has a nonzero $x_{i}^{a_{i}+d_{i}} y_{i}^{b_{i}}$ term with $\left(a_{i}+d_{i}, b_{i}\right) \not \equiv 0(m)$. The essential measures considered are a nonnegative integer $\left[b_{i}, c_{i}\right]$ in the case of $R_{i}$-type $\left(a_{i}, b_{i}, c_{i}\right)$, and a nonnegative integer $d_{i}$ in the case of antitype. The measure associated to $f_{i}(Z) \in R_{i}[Z]$ is either $\left[b_{i}, c_{i}\right]$ or $d_{i}$, computed from viewing $f_{i}(Z)$ as an appropriate $R_{i}$-type or $R_{i}$-antitype. Even the choice of $a_{i}$ and $b_{i}$ in an $R_{i}$-type or $R_{i}$-antitype is subtle. They may not be the highest powers of $x_{i}$ and $y_{i}$ which divide $f_{i}(0)$. We have that $\left[b_{i+1}, c_{i+1}\right] \leq\left[b_{i}, c_{i}\right]$ and $d_{i+1} \leq d_{i}$ under a quadratic transform. After an appropriate sequence of quadratic transforms, which depends on the type of valuation $\nu$ being considered, one of these numbers must drop. The function $[b, c]$ is used instead of $c$ since we may have $c_{i+1}>c_{i}$.

Finally, we consider the proof that $f(Z)$ is $R_{i}$-permissible for all $i \gg 0$, which is the statement of Theorem 7.2. The question reduces to showing (in Theorem 5.7) that if $L$ is a $p$-extension of $K$ and $\nu$ does not split in $L$, then for $n \gg 0, \operatorname{ord}_{R_{n}}$ does not split in $L$ and $\operatorname{ord}_{\left(R_{n}\right)_{x_{n} R_{n}}}$ is totally ramified in $L$.

A valuation $\omega$ of $K$ does not split in an algebraic extension $L$ if the integral closure $W$ of the valuation ring $V_{\omega}$ of $\omega$ in $L$ has only one maximal ideal. If, further, the residue field of $W$ over the residue field of $V_{\omega}$ is purely inseparable, then $\omega$ is totally ramified in $L$.

The proof of Theorem 5.7 follows easily once we have established it for the case when $L$ is an Artin-Schreier extension of $K$. For general $p$-extensions $L$, we deduce the result by knowing that it is true for all Artin-Schreier extensions of $K$ contained in $L$.

In the case of an Artin-Schreier extension, we have that $L=K(z)$ where

$$
f(Z)=Z^{p}+G^{p-1} Z+F
$$

with $F, G \in R$ is the minimal polynomial of $z$ over $K$. Direct computations are given in Sections 4 and 5 for this case. In Section 4, A proof of reduction of order is given for the Artin Schreier case, which is an essential component in Abhyankar's first (nonembedded) proof of resolution of surfaces [1]. 
A dictionary between the results in this paper and Abhyankar's original proof. The material in Section 3 on the relation of splitting type and ramification type of a polynomial to splitting and ramification of a valuation is from the paper [5]. Section 4 is a simplification of the proof in [2]. The main result Theorem 4.1 of this section is stated in [2] and in Section 9 of [1]. Theorem 5.1 of Section 5 is a case of a more general theorem proven in [4]. The final analysis of Artin-Schreier extensions leading up to Theorem 5.7 is a summary of results from [5]. The statement and proof of Theorem 5.7 is Theorem 4.23 [5]. Section 6 on polynomials is a summary of some of the results of Section 6 of [5]. Section 7, the degree $p^{t}$ case, is a simplification of proofs from [5]. The main theorems $7.2,7.7$ and 7.8 are cases of Theorems 5.4 [5], and cases of Theorems 5.3 and 5.5 [5]. Section 8 on reduction of order of a polynomial is a survey of results from [6]. Theorem 8.1 is Theorem 1.1 of [6]. It is also stated as (5.1) in [7]. Section 9, which proves local uniformization and global resolution of singularities, covers results of Chapters 1 and 2 of [7]. While the general method and outline of proof are the same in section 9 and in the book [7], our proofs are substantially simpler. Lemma 9.1 (on tangent cones and approximate manifolds) is stated as (3.10.1) and (4.4) in [7]. Lemma 9.9 and Lemma 9.10 are cases of (3.10.4) and (3.10.6) of [7]. Theorem 9.7 and Corollary 9.8 are versions of (5.2.1) and (5.2.4) [7]. The global resolution theorems Theorem 9.12, 9.14 and 9.15 are versions of results stated in Section 9 of [7].

2. Notation. The nonnegative integers will be denoted by $\mathbb{N}$. The positive integers will be denoted by $\mathbb{Z}_{+}$. Suppose that $i, j, p \in \mathbb{Z}$. We will write $(i, j) \equiv 0(p)$ if $p$ divides both $i$ and $j$.

We will write $M(A)$ for the maximal ideal of a quasi local ring ( $A$ has a unique maximal ideal, but $A$ might not be noetherian).

Suppose that $A$ is a local domain (A has a unique maximal ideal, and $A$ is noetherian) with quotient field $K$. Suppose that $\nu$ is a valuation of $K$. We will write $V_{\nu}$ for the valuation ring of $\nu$. We will say that $\nu$ dominates $A$ if $V_{\nu}$ dominates $A$; that is, $A \subset V_{\nu}$ and $M\left(V_{\nu}\right) \cap A=M(A)$, or equivalently, $\nu$ is nonnegative on $A$ and is positive on $M(A)$. We will say that $\nu$ is 0-dimensional if $\left.V_{\nu} / M\left(V_{\nu}\right)\right)$ is an algebraic extension of $A / M(A)$.

Suppose that $R$ is a regular local ring. For $f \in R$, we will write $\operatorname{ord}_{R}(f)$ for the $M(R)$-adic value of $f$; that is the largest power of $M(R)$ which contains $f$. When there is no danger of confusion, we may write ord $(f)$ for $\operatorname{ord}_{R}(f)$. Suppose that $x_{1}, \ldots, x_{n}$ is a regular system of parameters in $R$. We will say that $f \in R$ is an $R$-monomial in $x_{1}, \ldots, x_{n}$ if there exist $a_{1}, \ldots, a_{n} \in \mathbb{N}$ and a unit $\delta \in R$ such that $f=x_{1}^{a_{1}} \cdots x_{n}^{a_{n}} \delta$. Suppose that $R_{1}$ is a local ring of the blow up of a prime ideal $P$ in $\operatorname{spec}(R)$, such that $R / P$ is a regular local ring. Then the regular local ring $R_{1}$ is called a monoidal transform of $R$. In the case that $P$ is the maximal ideal of $R, R_{1}$ is called a quadratic transform of $R$. If $\nu$ is a valuation of $K$ which dominates $R$. we say that $R \rightarrow R_{1}$ is a monoidal transform along $\nu$ if $\nu$ dominates $R_{1}$.

Suppose that $X$ is a projective variety, over an algebraically closed field $\bar{k}$, with function field $\bar{k}(X)$. A valuation $\nu$ of $\bar{k}(X)$ is a valuation of the algebraic function field $\bar{k}(X)$ such that the valuation ring $V_{\nu}$ contains $\bar{k} . \nu$ is 0 -dimensional if $V_{\nu} / M\left(V_{\nu}\right)=\bar{k}$. The center of $\nu$ on $X$ is the unique point $p \in X$ such that $V_{\nu}$ dominates the local ring $\mathcal{O}_{X, p}$ (which exists by the valuative criterion for properness). If $\nu$ is zero dimensional, $p$ must be a closed point of $X$. Suppose that $X$ is nonsingular, and $Y$ is a codimension one subvariety of $X$. Suppose that $p \in Y$. We will say that $f=0$ is a local equation of $Y$ at $p$ if $\mathcal{I}_{Y, p}=f \mathcal{O}_{X, p}$, where $\mathcal{I}_{Y}$ is the ideal sheaf of $Y$ in $X$. We will say that $f=0$ 
is a formal local equation of $Y$ at $p$ if $\widehat{\mathcal{I}_{Y, p}}=f \widehat{\mathcal{O}_{X, p}}$, where $\widehat{\mathcal{O}_{X, p}}$ is the completion of $\mathcal{O}_{X, p}$ with respect to its maximal ideal.

DEFINITION 2.1. Let $S_{0}$ be a two dimensional regular local ring, containing an algebraically closed field $\bar{k}$ of characteristic $p$ which is isomorphic to the residue field of $S_{0}$. Let $K$ be the quotient field of $S_{0}$. Let $\nu$ be a 0 -dimensional valuation of $K$ which dominates $S_{0}$. Let

$$
S_{0} \rightarrow S_{1} \rightarrow \cdots \rightarrow S_{n} \rightarrow \cdots
$$

be the infinite sequence of quadratic transforms along $V$. Suppose that $x_{0}, y_{0}$ are regular parameters in $S_{0}$. For $n \geq 0$, the canonical parameters of $S_{n}$ determined by $x_{0}, y_{0}$ are the regular parameters $x_{n}, y_{n}$ in $S_{n}$ defined recursively by

$$
\begin{gathered}
x_{i}=x_{i+1}, y_{i}=x_{i+1}\left(y_{i+1}+\alpha_{i+1}\right) \text { with } \alpha_{i+1} \in \bar{k} \text { or }, \\
x_{i}=x_{i+1} y_{i+1}, y_{i}=y_{i+1} .
\end{gathered}
$$

The following Lemma is Lemma 1.2 [6].

LEMMA 2.2. Let $S_{0}$ be a two dimensional regular local ring, containing an algebraically closed field $\bar{k}$ of characteristic $p>0$ which is isomorphic to the residue field of $S_{0}$. Let $K$ be the quotient field of $S_{0}$. Let $\nu$ be a valuation of $K$ which dominates $S_{0}$ and is rational nondiscrete (the value group of $\nu$ is order isomorphic to a subset of $\mathbb{Q}$ which is not isomorphic to $\mathbb{Z}$ ). Suppose that $x_{0}, y_{0}$ are regular parameters in $S_{0}$. Then $\nu$ is 0-dimensional. Further, in the sequence (11) of quadratic transforms along $\nu$, transforms of the type of (12) with $\alpha_{i+1} \neq 0$ occur infinitely many times, and transforms of the type of (13) occur infinitely many times.

If $g(Z)=b_{n}+b_{n-1} Z+\cdots+b_{0} Z^{n} \in R[Z]$ with $b_{i} \in R$ is a polynomial, we will write $\operatorname{ord}_{R}(g)=r$ if $r=\min \left\{\operatorname{ord}_{R}\left(b_{i}\right)+n-i\right\}$.

Definition 2.3. Suppose that $R$ is a ring and $f(Z) \in R[Z]$ is a polynomial. An $R$-translate $f^{\prime}(Z)$ of $f(Z)$ is a polynomial $f^{\prime}(Z) \in R[Z]$ defined by $f^{\prime}(Z)=f(Z+r)$ for some $r \in R$.

We will make free use of the basic theorems of embedded resolution of ideals in a regular local ring of dimension two (c.f. Sections 3.5, 4.2 [17]) which we will call "embedded principalization of ideals" in a two dimensional regular local ring. In particular, we will frequently use the following result.

Lemma 2.4. Let assumptions be as in Definition 2.1. Suppose that $\nu$ is a rational nondiscrete valuation of $K$ which dominates $S_{0}$, and $F \in R$. Then there exists $n_{0}$ such that for all $n \geq n_{0}$ we have that $F=\delta_{n} x_{n}^{a_{n}}$ where $\delta_{n} \in S_{n}$ is a unit and $a_{n} \in \mathbb{N}$.

We will also make use of the standard properties of excellent rings, which are proven in Scholie IV.7.8.3 [22].

3. Nonsplitting. In this section we summarize some properties of splitting and nonsplitting of valuations.

Definition 3.1. Let $R$ be a normal quasi local domain with quotient field $K$. Let $L$ be an algebraic extension of $K$ and let $S$ be the integral closure of $R$ in $L$. We 
say that $R$ splits in $L$ if $S$ is not quasi local. We say that $R$ is totally ramified in $L$ if $R$ does not split in $L$ and $S / M(S)$ is purely inseparable over $R / M(R)$.

Given a valuation $\nu$ of a field $K$ and an algebraic extension $L$ of $K$ we say that $\nu$ splits in $L$ if $V_{\nu}$ splits in $L$. We say that $\nu$ is totally ramified in $L$ if $V_{\nu}$ is totally ramified in $L$.

Lemma 3.2. Suppose that $R$ is a normal quasi-local domain with quotient field $K, \nu$ is a valuation of $K$ which dominates $R$ and $L$ is an algebraic extension of $K$ such that $R$ splits in $L$. Then $\nu$ splits in $L$.

Proof. The valuation ring $V_{\nu}$ of $\nu$ is the directed union of the local rings $\left\{S_{j}\right\}$, obtained by blowing up a 2-generated ideal $I_{j}$ in $R$ and taking the local ring of the center of $\nu$.

Suppose that $A_{1}$ and $A_{2}$ are two different normal quasi local domains, which dominate $R$ and are localizations of the integral closure of $R$ in $L$. For $i=1,2$ let $B_{i}$ be a directed union of quasi local rings $T_{i j}$ such that $T_{i k}$ dominates $T_{i j}$ if $k>j$ and $T_{i j}$ is a local ring of the blow up of $I_{j} A_{i}$ which dominates $S_{j}$. Then $B_{i}$ are distinct quasi local rings which dominate $V_{\nu}$. By Theorem 5, Section 4, Chapter VI [47], there exist valuation rings $V_{1}$ and $V_{2}$ with quotient field $L$ which dominate $B_{1}$ and $B_{2}$ respectively.

$V_{1}$ and $V_{2}$ are distinct since $V_{1}$ dominates $A_{1}$ and $R$ and $V_{2}$ dominates $V_{2}$ and $R$. Since each $V_{i}$ dominates $V_{\nu}$ and $V_{i} \cap K$ is a valuation ring, $V_{1} \cap K=V_{2} \cap K=V_{\nu}$ by Lemma 2.31 [3]. By Lemma 2.37 [3], we have that $V_{1}$ and $V_{2}$ are distinct local rings of the integral closure of $V_{\nu}$ in $L$ which dominate $V_{\nu}$.

Definition 3.3. Let $\nu$ be a valuation of a field $K$, and let $K[Z]$ be a polynomial ring over $K$. Suppose that $f(Z) \in K[Z]$ is monic of degree $m>0$. We then have an expansion

$$
f(Z)=Z^{m}+\sum_{i=1}^{m-1} f_{i} Z^{m-i}+F
$$

with $F, f_{1}, \ldots, f_{m-1} \in K$. We say that $f(Z)$ is of prenonsplitting-type relative to $\nu$ if there exist $t_{i} \in V_{\nu}$ such that $f_{i}^{m}=t_{i} F^{i}$ for $0<i<m$.

We say that $f(Z)$ is of preramified-type relative to $\nu$ if the following three conditions hold:

1. $f(Z)$ is of prenonsplitting-type relative to $\nu$,

2. $V_{\nu} / M\left(V_{\nu}\right)$ is of characteristic $p \neq 0$ and $m$ is a power of $p$.

3. If there exists $G \in K$ and a unit $G^{\prime} \in V_{\nu}$ such that $F=G^{\prime} G^{m}$, then there exists $t_{i} \in M\left(V_{\nu}\right)$ such that $f_{i}^{m}=t_{i} F^{i}$ for $0<i<m$.

We say that $f(Z)$ is of nonsplitting-type relative to $\nu$ provided every $K$-translate of $f(Z)$ is of nonsplitting-type relative to $\nu$. We say that $f(Z)$ is of ramified-type relative to $\nu$ provided every $K$-translate of $f(Z)$ is of preramified-type relative to $\nu$.

The following Lemmas 3.4, 3.5 and 3.7 are proven in Lemmas 2.3, 2.5 and 2.10 of [5]. The proofs are by Galois theory.

LEMMA 3.4. Let $K$ be a field, let $L$ be a normal extension of $K$, let $\nu$ be a valuation of $K$ such that $\nu$ does not split in $L$, and let $f(Z)$ be a monic polynomial of degree $m>0$ in $K[Z]$ such that $f(Z)$ is irreducible in $K[Z]$ and $f(z)=0$ for some $z \in L$. Then $f(Z)$ is of nonsplitting type relative to $\nu$. 
Lemma 3.5. Let $K$ be a field, let $L$ be a normal extension of $K$, let $\nu$ be a valuation of $K$ such that $V_{\nu} / M\left(V_{\nu}\right)$ is of characteristic $p>0$ and $\nu$ is totally ramified in $L$, and let $f(Z) \in K[Z]$ be a monic polynomial of degree $m=p^{n}$, where $n$ is a positive integer, such that $f(Z)$ is irreducible in $K[Z]$ and $f(z)=0$ for some $z \in L$. Then $f(Z)$ is of ramified type relative to $\nu$.

Definition 3.6. A p-extension of a field $K$ of characteristic $p>0$ is a normal extension $L$ of $K$ such that $[L: K]=p^{n}$ for some $n \in \mathbb{N}$. A p-cyclic extension $K$ is a normal extension $L$ of $K$ such that $[L: K]=p$.

Lemma 3.7. Let $R$ be a normal quasilocal domain with quotient field $K$ and let $L$ be a p-extension of $K$. Then $R$ does not split (respectively $R$ is totally ramified) in $L$ if and only if for every subfield $K^{*}$ of $L$ which is a separable $p$-cyclic extension of $K$ we have that $R$ does not split (respectively $R$ is totally ramified) in $K^{*}$.

4. The Artin-Schreier Case. In this section, we prove the following theorem.

THEOREM 4.1. Let $K$ be a two dimensional algebraic function field over an algebraically closed field $\bar{k}$ of characteristic $p \neq 0$, let $K^{*}$ be a Galois extension of $K$ of degree $p$, and let $\nu$ be a rational nondiscrete valuation of $K$ having only one extension $\nu^{*}$ to $K^{*}$. Assume that $\nu$ can be uniformized. Then $\nu^{*}$ can be uniformized.

This theorem is stated in Theorem 4 of [1] (and later in [2]) and is a critical part of Abhyankar's proof of local uniformization of a valuation of a two dimensional algebraic function field over an algebraically closed field. Abhyankar makes use of ramification theory to reduce to the case of Theorem 4.1.

The statement " $\nu$ can be uniformized" means that there exists a regular local ring $R$, with quotient field $K$, such that $R$ is a localization of a finite type $\bar{k}$-algebra ( $R$ is an algebraic local ring of $K$ ) and $\nu$ dominates $R$. The statement that $\nu$ has only one extension to $K^{*}$ means that $\nu$ does not split in $K^{*}$ (Section 3). The statement that $\nu$ is a rational nondiscrete valuation means that the value group of $\nu$ is (order isomorphic to) a subset of $\mathbb{Q}$ which is not isomorphic to $\mathbb{Z}$.

Suppose that $R$ is a regular algebraic local ring of $K$ which is dominated by $\nu$. Let $x, y$ be a regular system of parameters in $R$. let $R_{1}$ be the local ring of the quadratic transform of $R$ which is dominated by $\nu$. Then $R_{1}$ has a regular system of parameters $x_{1}, y_{1}$ of one of the following types:

$$
x=x_{1}, y=x_{1}\left(y_{1}+\alpha_{1}\right)
$$

with $\alpha_{1} \in \bar{k}$, or

$$
x=x_{1} y_{1}, y=y_{1} .
$$

We can continue to blow up maximal ideals to construct a sequence of regular algebraic local rings,

$$
R \rightarrow R_{1} \rightarrow R_{2} \rightarrow \cdots
$$

along (dominated by) $\nu$, where each $R_{i+1}$ has regular parameters $\left(x_{i+1}, y_{i+1}\right)$ of one of the following types:

$$
x_{i}=x_{i+1}, y_{i}=x_{i+1}\left(y_{i+1}+\alpha_{i+1}\right)
$$


with $\alpha_{i+1} \in \bar{k}$, or

$$
x_{i}=x_{i+1} y_{i+1}, y_{i}=y_{i+1} .
$$

We will also allow interchanging of the variables $x_{i}, y_{i}$.

The fact that $\nu$ is rational nondiscrete tells us that we must obtain a form (17) with $\alpha_{i+1} \neq 0$ infinitely many times in the sequence, and we must obtain a form (18) infinitely many times in the sequence.

We know from "embedded principalization of ideals" in regular local rings of dimension two, and since $\nu$ is rational nondiscrete, that if $f \in R$, then there exists an index $i$ in the sequence (16) such that $f=x_{i}^{n} \delta$ where $n \in \mathbb{N}$ and $\delta$ is a unit in $R_{i}$

We now introduce a construction which will be used in the proof of the theorem. Since $K^{*}$ is a galois extension of $K$ of degree $p$, it is an Artin-Schreier extension. Thus $K^{*}=K\left[\bar{z}^{\prime}\right]$ where the minimal polynomial $g^{\prime}(z)$ of $\bar{z}^{\prime}$ over $K$ has the form $g^{\prime}(z)=z^{p}+z+d$. Since $\omega$ can be uniformized, there exists a regular algebraic local ring $R$ of $K$ such that $\omega$ dominates $R$. Write $d=\frac{a}{b}$ where $a, b \in R$. Setting $\bar{z}=b \bar{z}^{\prime}$, we obtain that $K^{*}=K[\bar{z}]$ and the minimal polynomial of $\bar{z}$ over $K$ has the form

$$
g(z)=z^{p}+h^{p-1} z+f
$$

where $h, f \in R$. The domain $S=R[\bar{z}] \cong R[z] /(g(z))$ is integral over $R$ and has the quotient field $K^{*}$. Thus $\nu^{*}$ is nonnegative on $S$. Let $T$ be the integral closure of $R$ in $K^{*}$. Since $\nu$ does not split in $K^{*}, T$ is a local ring. The center of $\nu^{*}$ on $S$ is the maximal ideal $m_{T} \cap S$. Let $N=M(R) R[z]+z R[z]$, a maximal ideal of $R[z]$.

We now make a fundamental observation.

LEMMA 4.2. Suppose that $x, y$ are a regular system of parameters in $R$, so that the polynomial ring $\bar{k}[x, y]$ is a subset of $R$. Suppose that $\operatorname{ord}(g(z))>0$. Then the center of $\nu^{*}$ on $S$ is the maximal ideal $(x, y, \bar{z})$.

Proof. The assumption that $\operatorname{ord}(g(z))>0$ implies that the ideal $m_{1}=(x, y, \bar{z})$ is a maximal ideal of $S$ which contracts to $M(R)$. But $m_{1}$ is then the unique maximal ideal which dominates $M(R)$, since $\nu$ does not split in $K^{*}$ (by Lemma 3.2).

We point out that our assumption of nonsplitting implies that $\operatorname{ord}(h)>0$ in (19). Otherwise, the residue of $g(z)$ in $R / M(R)[z] \cong \bar{k}[z]$ would be an Artin Schreier polynomial, and there would be $p$ distinct maximal ideals in $S$ which contract to $M(R)$, which we know cannot happen (it would contradict the assumption that $\nu$ does not split in $\left.K^{*}\right)$.

We will perform 3 types of operations on the polynomial ring $R[z]$, which induce birational transformations of $S$. Suppose that $x, y$ are regular parameters in $R$. Then there is a natural inclusion of the polynomial ring $\bar{k}[x, y]$ into $R$.

The first and simplest operation is to "clean" the coefficients of $g(z)$. Suppose that $A(x, y) \in \bar{k}[x, y]$. We can make a change of variables in $R[z]$, replacing $z$ with $z^{\prime}=z-A(x, y)$. We then set $g^{\prime}\left(z^{\prime}\right)=g\left(z^{\prime}+A(x, y)\right) \in R\left[z^{\prime}\right]$. Set $\bar{z}^{\prime}=\bar{z}-A(x, y)$. We then have that $S=R\left[\bar{z}^{\prime}\right] \cong R\left[z^{\prime}\right] /\left(g^{\prime}\left(z^{\prime}\right)\right)$.

The most basic case of this transformation is to make $\operatorname{ord}\left(g^{\prime}\left(z^{\prime}\right)\right)>0$. There exists $\alpha \in \bar{k}$ such that $f-\alpha \in M(R)$. Set $z^{\prime}=z+\sqrt[p]{\alpha}$. Then, since $\operatorname{ord}(h)>0$, we have that $\operatorname{ord}\left(g^{\prime}\left(z^{\prime}\right)\right)>0$. More generally, we can view $f$ as an element of the completion $\hat{R} \cong \bar{k}[[x, y]]$ of $R$, and "clean" to remove $p$-th powers from $f$ be making substitutions $z^{\prime}=z-A(x, y)$.

The second type of operation is to perform a quadratic transform $R \rightarrow R_{1}$ along $\nu$. The regular local ring $R_{1}$ has a regular system of parameters $x_{1}, y_{1}$ defined by 
(14) or (15). We view $g(z)$ as an (irreducible) element of the polynomial ring $R_{1}[z]$. Set $S_{1}=R_{1}[\bar{z}] \cong R_{1}[z] /(g(z))$. $S_{1}$ is a birational extension of $S$, such that $\nu^{*}$ is nonnegative on $S_{1}$.

Using quadratic transformations of $R$, we can make $h$ a monomial. By "embedded principalization of ideals" in $R$, we can construct a sequence $R \rightarrow R_{i}$ of quadratic transformations (16) along $\nu$, such that in $R_{i}[z]$, we have

$$
g(z)=z^{p}+\left(x_{i}^{a_{i}} y_{i}^{b_{i}}\right)^{(p-1)} \delta_{i} z+f_{i}
$$

where $x_{i}, y_{i}$ are a regular system of parameters in $R_{i}, \delta_{i}, f_{i} \in R_{i}$ and $\delta_{i}$ is a unit in $R_{i}$. We may thus assume that this forms holds in $R$, so that

$$
g(z)=z^{p}+\left(x^{a} y^{b}\right)^{(p-1)} \delta z+f .
$$

Suppose that (21) holds and $R \rightarrow R_{1}$ is a quadratic transformation along $\nu$. Then

$$
a_{1}=a+b, b_{1}=b
$$

if (14) holds with $\alpha_{1}=0$,

$$
a_{1}=a+b, b_{1}=0
$$

if (14) holds with $\alpha_{1} \neq 0$, and

$$
a_{1}=a, b_{1}=a+b
$$

if (15) holds.

The third type of operation is to make a monomial substitution for $z$. Suppose that $s, t \in \mathbb{N}$ are such that $x^{s p} y^{t p}$ divides $f$ in $R$, with $s \leq a, t \leq b$. Define $z_{1}$ by

$$
z=x^{s} y^{t} z_{1}
$$

Define

$$
g_{1}\left(z_{1}\right)=\frac{g\left(x^{s} y^{t} z_{1}\right)}{x^{s p} y^{t p}} .
$$

The element $g_{1}\left(z_{1}\right)$ is in the polynomial ring $R\left[z_{1}\right]$. Substituting into (21), we see that

$$
g_{1}\left(z_{1}\right)=z_{1}^{p}+\left(x^{a_{1}} y^{b_{1}}\right)^{(p-1)} \delta z_{1}+f_{1},
$$

where $a_{1}=a-s, b_{1}=b-t$ and

$$
f_{1}=\frac{f}{x^{s p} y^{t p}} \in R .
$$

Define $\bar{z}_{1} \in K^{*}$ by

$$
\bar{z}=x^{s} y^{t} \bar{z}_{1} .
$$

Let $S_{1}=R\left[\bar{z}_{1}\right] \cong R\left[z_{1}\right] /\left(g_{1}\left(z_{1}\right)\right) . S_{1}$ is a birational extension of $S$. The valuation $\nu^{*}$ is nonnegative on $S_{1}$ since $S_{1}$ is integral over $R$.

We will construct sequences of operations of these three types. Composing the three operations will give us the data of a birational extension of regular local rings 
$R \rightarrow R_{1}$, with a regular system of parameters $x_{1}, y_{1}$ in $R_{1}$, a polynomial ring $R_{1}\left[z_{1}\right]$, an irreducible polynomial $g_{1}\left(z_{1}\right) \in R_{1}\left[z_{1}\right]$ which has the form

$$
g_{1}\left(z_{1}\right)=z_{1}^{p}+\left(x_{1}^{a_{1}} y_{1}^{b_{1}}\right)^{(p-1)} \delta_{1} z_{1}+f_{1}
$$

where $\delta_{1}, f_{1} \in R_{1}$ and $\delta_{1}$ is a unit. We further have a birational extension $S \rightarrow S_{1}=$ $R_{1}\left[\bar{z}_{1}\right] \cong R_{1}\left[z_{1}\right] /\left(g_{1}\left(z_{1}\right)\right)$, where $\nu^{*}$ is nonnegative on $S_{1}$.

Our choice of regular parameters $x, y$ in $R$ gives us an identification of $\hat{R}$ with the power series ring $\bar{k}[[x, y]]$. We then have an expansion

$$
f=\sum_{i, j \in \mathbb{N}} f_{i, j} x^{i} y^{j}
$$

with $f_{i, j} \in \bar{k}$. We also have an associated series $f_{1} \in \bar{k}\left[\left[x_{1}, y_{1}\right]\right]$, with coefficients $\left(f_{1}\right)_{i, j} \in \bar{k}$.

We will summarize the above data by saying that $(R, g),\left(R_{1}, g_{1}\right)$ are states (with associated equations (21) and (22)), and call such a sequence of operations a transformation from $(R, g)$ to $\left(R_{1}, g_{1}\right)$. We will also refer to states such as $\left(R^{\prime}, g^{\prime}\right)$, where it is understood that the complete set of data will be written as $S^{\prime}, x^{\prime}, y^{\prime}, z^{\prime}, f^{\prime}, a^{\prime}, b^{\prime}$, etc. We will also find it convenient at one point to interchange the variables $x$ and $y$ in $R$, and then make the obvious change of notation in the state $(R, g)$.

We will say that a state $(R, g)$ is resolved if $0<\operatorname{ord}(g)<p$.

The following two lemmas, Lemma 4.3 and Lemma 4.4, are completely worked out in (5.1), (5.2) and (5.3) of [2]. The proofs are straightforward, but somewhat technical.

Suppose that $i, j \in \mathbb{Z}$. Recall that $(i, j) \equiv 0(p)$ if $p$ divides both $i$ and $j$.

Lemma 4.3. Suppose that $(R, g)$ is a state. Then there exists $A(x, y) \in \bar{k}[x, y]$ and a transformation $(R, g) \rightarrow\left(R_{1}, g_{1}\right)$ obtained by setting $z_{1}=z-A(x, y)$ such that $g_{1}\left(z_{1}\right)=g\left(z_{1}+A(x, y)\right)$ has the form (22), with $\left(f_{1}\right)_{i, j}=0$ for all $(i, j)$ such that $(i, j) \equiv 0(p)$ and $i+j \leq p \max \{a, b\}$.

This is (5.3) [2].

Lemma 4.4. Suppose that $n \in \mathbb{Z}_{+}$and $(R, g)$ is a state such that $a>0$ (in (21)) and there exist $l, m \in \mathbb{N}$ with $l<p,(l, m) \not \equiv 0(p), f_{l, m} \neq 0$, and $f_{i, j}=0$ for all $i<l$. Then there exists $A(x, y) \in \bar{k}[x, y]$ and a transformation $(R, g) \rightarrow\left(R_{1}, g_{1}\right)$ obtained by setting $z_{1}=z-A(x, y)$ such that $g_{1}\left(z_{1}\right)=g\left(z_{1}+A(x, y)\right)$ has the form (22), with $\left(f_{1}\right)_{l, m} \neq 0,\left(f_{1}\right)_{i, j}=0$ for all $i<l$ and $\left(f_{1}\right)_{i, j}=0$ for all $(i, j)$ such that $(i, j) \equiv 0(p)$ and $i+j \leq p \max \{a, b\}$.

This is (5.2) of [2].

Lemma 4.5. Suppose that $n \in \mathbb{Z}_{+}$and $(R, g)$ is a state such that $\max \{a, b\}=n$ in (21). (The number $n$ is necessarily $\geq 1$, as remarked after Lemma 4.2). Then there exists a transformation of states $(R, g) \rightarrow\left(R_{1}, g_{1}\right)$ such that one of the following holds in (22):

1. $\max \left\{a_{1}, b_{1}\right\}<n$ or

2. $\max \left\{a_{1}, b_{1}\right\}=n$ and there exists $l, m \in \mathbb{N}$ with $l+m<n p$ such that $\left(f_{1}\right)_{l, m} \neq$ 0 , and $\left(f_{1}\right)_{i, j}=0$ whenever $(i, j) \equiv 0(p)$ with $i+j \leq l+m$.

Proof. By Lemma 4.3, we can make a a change of variables (a transformation of the first type) in $z$, to achieve that $f_{i, j}=0$ for all $(i, j)$ such that $(i, j) \equiv 0(p)$ 
with $i+j \leq p n$. Suppose that 2 does not hold. Then we have that $\operatorname{ord}(f) \geq n p$. Perform the quadratic transform $R \rightarrow R_{1}$ along $\nu$. Let $x_{1}, y_{1}$ be the regular system of parameters in $R_{1}$ determined by this quadratic transform. If $x_{1}, y_{1}$ are of of the type (14), then define $z=x_{1}^{n} z_{1}$. If $x_{1}, y_{1}$ are of type (15), then define $z=y_{1}^{n} z_{1}$. Since $\operatorname{ord}(f) \geq n p$ and $a+b \geq n$, this defines a transformation $(R, g) \rightarrow\left(R, g_{1}\right)$.

In the case that $x=x_{1}, y=x_{1}\left(y_{1}+\alpha_{1}\right), z=x_{1}^{n} z_{1}$, we have that

$$
g_{1}\left(z_{1}\right)=z_{1}^{p}+\left(x_{1}^{(a+b)-n}\left(y_{1}+\alpha_{1}\right)^{b}\right)^{p-1} \delta z_{1}+\frac{f}{x_{1}^{n p}},
$$

with a similar expression if $x=x_{1} y_{1}, y=y_{1}, z=y_{1}^{n} z_{1}$. We see that

$$
\left(a_{1}, b_{1}\right)= \begin{cases}(a+b-n, 0) & \text { if } x=x_{1}, y=x_{1}\left(y_{1}+\alpha_{1}\right) \text { with } \alpha_{1} \neq 0 \\ (a+b-n, b) & \text { if } x=x_{1}, y=x_{1} y_{1} \\ (a, a+b-n) & \text { if } x=x_{1} y_{1}, y=y_{1} .\end{cases}
$$

We thus have $\max \left\{a_{1}, b_{1}\right\} \leq \max \{a, b\}=n$.

If the conclusions of the lemma do not hold for $\left(R_{1}, g_{1}\right)$, then we may repeat the above process. Assume that after a finite number of iterations of this process we do not achieve the conclusions of the theorem. Since $\nu$ is nondiscrete rational, we must eventually perform a quadratic transform of the type (17) with $\alpha_{i+1} \neq 0$. Then we have $\left(a_{i+1}, b_{i+1}\right)=(n, 0)$. Since we do not achieve a reduction of $\max \left\{a_{i+1}, b_{i+1}\right\}$ in the next iteration, we must perform a quadratic transform of the type of (18), and we have $\left(a_{i+2}, b_{i+2}\right)=(n, 0)$. Thus all quadratic transforms that we perform must be of the type (18) from then on, which is impossible since $\nu$ is rational and nondiscrete.

Lemma 4.6. Suppose that $n \in \mathbb{Z}_{+}$and $(R, g)$ is a state such that $\max \{a, b\}=n$ in (21) and there exists $l, m \in \mathbb{N}$ with $l+m<n p$ such that $f_{l, m} \neq 0$, and $f_{i, j}=0$ whenever $(i, j) \equiv 0(p)$ with $i+j \leq l+m$. Then there exists a transformation of states $(R, g) \rightarrow\left(R_{1}, g_{1}\right)$ such that $a_{1}>0$ and there exist $l_{1}, m_{1} \in \mathbb{N}$ with $l_{1}<p, m_{1}<n p$, $\left(l_{1}, m_{1}\right) \not \equiv 0(p),\left(f_{1}\right)_{l_{1}, m_{1}} \neq 0$, and $\left(f_{1}\right)_{i, j}=0$ for all $i<l_{1}$.

Proof. Let $d=\operatorname{ord}(f)$. We have $d \leq l+m$. Let $q$ be the greatest integer such that $q p \leq d$. By our assumptions, $q \leq n-1$. After possibly interchanging $x$ and $y$ (the assumptions of Lemma 4.6 are symmetric in $x$ and $y$ ), we may assume that $\omega(y) \geq \omega(x)$.

Set $l_{1}=d-q p<p$. Let

$$
m_{1}=\max \left\{j \mid f_{d-j, j} \neq 0\right\} .
$$

By our assumptions, $\left(d-m_{1}, m_{1}\right) \not \equiv 0(p)$. Thus $\left(l_{1}, m_{1}\right) \not \equiv 0(p)$. We further have $m_{1} \leq l+m<n p$.

We now perform the quadratic transform $R \rightarrow R_{1}$ along $\nu . \quad R_{1}$ has regular parameters $x_{1}, y_{1}$ defined by

$$
x=x_{1}, y=x_{1}\left(y_{1}+\alpha_{1}\right)
$$

for some $\alpha_{1} \in \bar{k}$. We have that $q p \leq d$ and $x_{1}^{d}$ divides $f$ in $R_{1}$. We now make the substitution $z=x_{1}^{q} z_{1}$ to construct a transformation of states $(R, g) \rightarrow\left(R_{1}, g_{1}\right)$. We have

$$
g_{1}\left(z_{1}\right)=z_{1}^{p}+\left(x_{1}^{a+b-q}\left(y_{1}+\alpha_{1}\right)^{b}\right)^{p-1} \delta z_{1}+f_{1}
$$


where

$$
f_{1}=\frac{f\left(x_{1}, x_{1}\left(y_{1}+\alpha_{1}\right)\right)}{x_{1}^{q p}} .
$$

We have that

$$
a_{1}=a+b-q \geq \max \{a, b\}-(n-1) \geq 1 \text {. }
$$

Further,

$$
\begin{aligned}
f_{1} & =x_{1}^{d-q p}\left(\sum_{i+j=d} f_{i, j}\left(y_{1}+\alpha_{1}\right)^{j}+x_{1} \Omega\right) \\
& =x_{1}^{l_{1}}\left(f_{d-m_{1}, m_{1}} y_{1}^{m_{1}}+\text { lower order terms in } y_{1}+x_{1} \Omega\right)
\end{aligned}
$$

for some $\Omega_{1} \in R_{1}$. Thus the conclusions of the lemma hold.

REMARK 4.7. We could have an increase $\max \left\{a_{1}, b_{1}\right\}>n$ in the state $\left(R_{1}, g_{1}\right)$ of the conclusions of Lemma 4.6.

Lemma 4.8. Suppose that $n \in \mathbb{Z}_{+}$and $(R, g)$ is a state such that

$a>0$ in (21), and there exist $l, m \in \mathbb{N}$ with $l<p, m<n p,(l, m) \not \equiv 0(p)$, $f_{l, m} \neq 0$, and $f_{i, j}=0$ for all $i<l$. Then there exists a transformation of states $(R, g) \rightarrow\left(R_{1}, g_{1}\right)$ such that one of the following holds:

1. $\left(R_{1}, g_{1}\right)$ is resolved, or

2. $\max \left\{a_{1}, b_{1}\right\}<n$ (in (22)) or

3. The assumptions of this lemma hold, with a reduction in $m$; that is, $a_{1}>0$ and there exist $l_{1}, m_{1} \in \mathbb{N}$ with $l_{1}<p, m_{1}<m<n p,\left(l_{1}, m_{1}\right) \not \equiv 0(p)$, $\left(f_{1}\right)_{l_{1}, m_{1}} \neq 0$, and $f_{i, j}=0$ for all $i<l_{1}$.

Proof. By Lemma 4.4, we can make a a change of variables (a transformation of the first type) in $z$, to achieve that $f_{l, m} \neq 0, f_{i, j}=0$ for all $i<l$ and $f_{i, j}=0$ for all $(i, j)$ such that $(i, j) \equiv 0(p)$ with $i+j \leq p \max \{a, b\}$. Assume that $\max \{a, b\} \geq n$ and $(R, g)$ is not resolved. Then $\operatorname{ord}(f) \geq p$. We must have that $m>0$, since $l<p$ and $\operatorname{ord}(f) \geq p$.

Perform a quadratic transform $R \rightarrow R_{1}$ along $\nu$. Let $x_{1}, y_{1}$ be the regular system of parameters in $R_{1}$ determined by this transformation.

Case I. Suppose that $\nu(y)<\nu(x)$, so that $R_{1}$ has regular parameters $x_{1}, y_{1}$ defined by $x=x_{1} y_{1}, y=y_{1}$. We have that $y_{1}^{p}$ divides $f$ in $R_{1}$ since ord $(f) \geq p$. We also have that $a+b \geq 1$. We may thus define a transformation $(R, g) \rightarrow\left(R_{1}, g_{1}\right)$ by the substitution $z=y_{1} z_{1}$. We have that

$$
g_{1}\left(z_{1}\right)=z_{1}^{p}+\left(x_{1}^{a} y_{1}^{a+b-1}\right)^{p-1} \delta z_{1}+f_{1}
$$

where

$$
f_{1}=\frac{f\left(x_{1} y_{1}, y_{1}\right)}{y_{1}^{p}}
$$

Let $l_{1}=l$ and $m_{1}=l+m-p<m$. Since $\left(f_{1}\right)_{i, j} \neq 0$ if and only if $f_{i, j-i+p} \neq 0$, we have that $\left(R_{1}, g_{1}\right)$ is resolved, or $\max \left\{a_{1}, b_{1}\right\}<n$ or the conclusion 3 of Lemma 4.8 holds for $\left(R_{1}, g_{1}\right)$ for $l_{1}, m_{1}$, with $m_{1}<m$. 
Case II. Suppose that $\nu(y) \geq \nu(x)$, so that $R_{1}$ has regular parameters $x_{1}, y_{1}$ defined by $x=x_{1}, y=x_{1}\left(y_{1}+\alpha_{1}\right)$ for some $\alpha_{1} \in \bar{k}$. Let $d=\operatorname{ord}(f)$. Let $q$ be the greatest integer such that $q p \leq d$. We have that $q p \leq l+m<(n+1) p$, so that

$$
q \leq n .
$$

Suppose that $i, j$ are such that $i+j \leq l+m$ and $(i, j) \equiv 0(p)$. Since $l+m<(n+1) p$, we have that $i+j \leq n p \leq p \max \{a, b\}$, so that $f_{i, j}=0$ by our assumptions.

We have that $x_{1}^{q p}$ divides $f$ in $R_{1}$ and $a+b \geq n \geq q$. We may thus define a transformation $(R, g) \rightarrow\left(R_{1}, g_{1}\right)$ by the substitution $z=x_{1}^{q} z_{1}$. We have that

$$
g_{1}\left(z_{1}\right)=z_{1}^{p}+\left(x_{1}^{a+b-q}\left(y_{1}+\alpha_{1}\right)^{b}\right)^{p-1} \delta z_{1}+f_{1}
$$

where

$$
f_{1}=\frac{f\left(x_{1}, x_{1}\left(y_{1}+\alpha_{1}\right)\right)}{x_{1}^{q p}} .
$$

We have that $a_{1}=a+b-q$ and $b_{1}=b$ if $\alpha_{1}=0, b_{1}=0$ if $\alpha_{1} \neq 0$. Let $x^{\bar{l}} y^{\bar{m}}$ be the term (with nonzero coefficient) of the expansion of $f$ in $\bar{k}[[x, y]]$ with $\bar{l}+\bar{m}=d$ with largest value of $\bar{m}$. By our assumptions, we must have that $\bar{l} \geq l, \bar{m} \leq m$, and $(\bar{l}, \bar{m}) \not \equiv 0(p)$. The monomial $x_{1}^{d-q p} y_{1}^{\bar{m}}$ appears (with nonzero coefficient) in $f_{1}$. Set $l_{1}=d-q p$ and $m_{1}=\bar{m}$.

If $\max \left\{a_{1}, b_{1}\right\} \geq n$, then we must have $a_{1}>0$. The assumptions of Lemma 4.8 then hold for $\left(R_{1}, g_{1}\right)$, with $m_{1} \leq m$. If $m_{1}<m$, then case 3 of the conclusions of the lemma hold, and we are done.

If $m_{1}=m$ (and $\left(R_{1}, g_{1}\right)$ is not resolved and $\max \left\{a_{1}, b_{1}\right\} \geq n$ ), then we repeat the algorithm of this lemma, applied to $\left(R_{1}, g_{1}\right)$. If we continue to iterate and not reach the conclusions of the lemma, then we must eventually reach the Case I, since $\omega$ is not discrete. This is then necessarily the last iteration of the algorithm, and the conclusions of the lemma are reached.

Lemma 4.9. Suppose that $n \in \mathbb{Z}_{+}$and $(R, g)$ is a state such that $a>0$ in (21), and there exist $l, m \in \mathbb{N}$ with $l<p, m<n p,(l, m) \not \equiv 0(p), f_{l, m} \neq 0$, and $f_{i, j}=0$ for all $i<l$. Then there exists a transformation of states $(R, g) \rightarrow\left(R_{1}, g_{1}\right)$ such that either $\left(R_{1}, g_{1}\right)$ is resolved, or $\max \left\{a_{1}, b_{1}\right\}<n$ (in (22)).

Proof. Lemma 4.9 follows from descending induction on $l$ in Lemma 4.8. $\square$

Proposition 4.10. Suppose that $n \in \mathbb{Z}_{+}$and $(R, g)$ is a state such that $\max \{a, b\}=n$ in (21). Then there exists a transformation of states $(R, g) \rightarrow\left(R_{1}, g_{1}\right)$ such that either $\left(R_{1}, g_{1}\right)$ is resolved or $\max \left\{a_{1}, b_{1}\right\}<n$ in (22).

Proof. The proposition follows from successive application (as necessary) of Lemmas 4.5, 4.6 and 4.9.

We now can easily finish the proof of Theorem 4.1. We start with a state $(R, g)$. By descending induction on $n$ in Proposition 4.10, we can constuct a transformation of states $(R, g) \rightarrow\left(R_{1}, g_{1}\right)$ such that $\left(R_{1}, g_{1}\right)$ is resolved. let $A=\left(S_{1}\right)_{\left(x_{1}, y_{1}, \bar{z}_{1}\right)} \cong$ $\left(R_{1}\left[z_{1}\right] /\left(g_{1}\right)\right)_{\left(x_{1}, y_{1}, z_{1}\right)}$ be the associated algebraic local ring of $K^{*}$ which is dominated by $\nu^{*}$.

We have that $0<\operatorname{ord}\left(g_{1}\right)<p$, so that $A$ is a hypersurface singularity of multiplicity less than $p$. We may now construct a birational extension $A \rightarrow B$ where $B$ is a regular algebraic local ring of $K^{*}$ dominated by $\nu^{*}$ using characteristic zero techniques. For instance, we can make a Tschirnhausen transformation to find a hypersurface of maximal contact. 
5. More on the Artin-Schreier Case. Suppose that $R$ is a regular local ring of dimension two, containing an algebraically closed field $\bar{k}$ of characteristic $p>0$ which is isomorphic to the residue field of $R$. Let $K$ be the quotient field of $R$, and let $\nu$ be a rational nondiscrete valuation of $K$ which dominates $R$. Let

$$
R \rightarrow R_{1} \rightarrow R_{2} \rightarrow \cdots \rightarrow R_{n} \rightarrow \cdots
$$

be the sequence of quadratic transforms along $\nu$. Suppose that $x, y$ are regular parameters in $R$. Recall (Definition 2.1) that $R_{i}$ has "canonical parameters" $x_{i}, y_{i}$, which are defined inductively by $x_{0}=x, y_{0}=y, x_{i-1}=x_{i}, y_{i-1}=x_{i}\left(y_{i}+\alpha_{i}\right)$ with $\alpha_{i} \in \bar{k}$ or by $x_{i-1}=x_{i} y_{i}, y_{i-1}=y_{i}$.

Looking back at our proof of Theorem 4.1, we see that the essential part of the proof can be restated as follows.

THEOREM 5.1. Suppose that $x, y$ are regular parameters in $R$, and suppose that $f(Z)=Z^{p}+g^{p-1} g^{\prime} Z+F \in R[Z]$ where $g^{\prime} \in R$ is a unit and $g, F \in R$. Let $z$ be $a$ root of $f(Z)=0$ in an extension field of $K$ and suppose that $\nu$ does not split in $K[z]$. Then there exists $n \in \mathbb{N}, u, v \in \mathbb{N}$, regular parameters $x^{\prime}, y^{\prime}$ in $R_{n}$, an $R_{n}$-monomial $s$ in $x^{\prime}, y^{\prime}$ and $s^{\prime} \in R_{n}$ such that if we set $f^{\prime}(Z)=s^{-p} f\left(s Z+s^{\prime}\right)$, we have that

$$
f^{\prime}(Z)=Z^{p}+\left(\left(x^{\prime}\right)^{u}\left(y^{\prime}\right)^{v}\right)^{p-1} \delta_{n} Z+F_{n}
$$

where $\delta_{n} \in R_{n}$ is a unit, $F_{n} \in R_{n}, u+v \geq 1$ and $0<\operatorname{ord}_{R_{n}} f^{\prime}(Z)<p$.

The following extension of Theorem 5.1 is proven in Proposition 27 [4]. The general strategy is similar to that of the proof of Proposition 4.10.

THEOREM 5.2. Suppose that $x, y$ are regular parameters in $R$, and suppose that $f(Z)=Z^{p}+\left(x^{\bar{u}} y^{\bar{v}}\right)^{p-1} \delta Z+F \in R[Z]$ where $\delta \in R$ is a unit, $F \in R$ and $\bar{u}, \bar{v} \in \mathbb{N}$. Let $z$ be a root of $f(Z)=0$ in an extension field of $K$ and suppose that $\nu$ does not split in $K[z]$. Then there exists $n \in \mathbb{N}, u, v \in \mathbb{N}$, regular parameters $x^{\prime}, y^{\prime}$ in $R_{n}$, an $R_{n}$-monomial $s$ in $x^{\prime}, y^{\prime}$ and $s^{\prime} \in R_{n}$ such that if we set $f^{\prime}(Z)=s^{-p} f\left(s Z+s^{\prime}\right)$, we have that

$$
f^{\prime}(Z)=Z^{p}+\left(\left(x^{\prime}\right)^{u}\left(y^{\prime}\right)^{v}\right)^{p-1} \delta_{n} Z+\left(x^{\prime}\right)^{a}\left(y^{\prime}\right)^{b} \varepsilon_{n}
$$

where $\delta_{n}, \varepsilon \in R_{n}$ are units, $u>0,0 \leq a<p, 0 \leq b<p,(a, b) \not \equiv 0(p)$ and if $b \neq 0$ then $v \neq 0$.

We require a version of this theorem where we do not make make operations of the third type. A rework of the above proof, or an argument starting with the conclusions of Theorem 5.2, as shown in Theorem 4.3 [5], proves the following theorem.

TheOREM 5.3. Suppose that $f(Z)=Z^{p}+\left(x^{\bar{u}} y^{\bar{v}}\right)^{p-1} \delta Z+F \in R[z]$ where $\bar{u}, \bar{v} \in \mathbb{N}$, $\delta \in R$ is a unit and $F \in R$. Suppose that $f(Z)$ is irreducible in $K[Z]$. Let $z$ be $a$ root of $f(Z)=0$ in an extension field of $K$ and suppose that $\nu$ does not split in $K[z]$. Then there exists $n \in \mathbb{N}$ and an $R_{n}$-translate $f_{n}(Z)$ of $f(Z)$ such that (after possibly interchanging $x_{n}$ and $y_{n}$ )

$$
f_{n}(Z)=Z^{p}+\left(x_{n}^{u} y_{n}^{v}\right)^{p-1} \delta_{n} Z+x_{n}^{a} y_{n}^{b} \varepsilon
$$

where $\delta_{n}, \varepsilon \in R_{n}$ are units, $(a, b) \not \equiv 0(p), 0 \leq a<u p$ and $b \leq v p$.

Definition 5.4. Suppose that $f(Z) \in R[Z]$ and $f(Z)=Z^{p}+G Z+F$ with $F, G \in R$ such that $G=g^{p-1} \delta$ where $\delta \in R$ is a unit and $g \in R$. 
1. $f(Z)$ is of standard type 1 relative to $(R, x, y)$ if $G=\left(x^{u} y^{v}\right)^{p-1} \delta$ where $\delta \in R$ is $a$ unit and $F=\gamma x^{a} y^{b}$ where $\gamma \in R$ is a unit, $(a, b) \not \equiv 0(p), 0 \leq a<$ up and $0 \leq b \leq v p$.

2. $f(Z)$ is of standard type 2 relative to $(R, x, y)$ if $G=\left(x^{u} y^{v}\right)^{p-1} \delta$ where $\delta \in R$ is a unit, $F=x^{a} y^{b} \tau$ where $\tau \in R, a<u p, b \leq v p, b \equiv 0(p)$ and $\operatorname{ord}_{R / x R}(\tau)=$ 1.

TheOREM 5.5. Suppose that $f(Z)=Z^{p}+G Z+F$ with $F, G \in R$ such that $G=g^{p-1} \delta$ where $\delta \in R$ is a unit and $g \in R$. Suppose that $f(Z)$ is irreducible in $K[Z]$. Let $z$ be a root of $f(Z)=0$ in an extension field of $K$ and suppose that $\nu$ does not split in $K[z]$. Then there exists a positive integer $m$ such that for every integer $n \geq m$ there exists an $R_{n}$-translate $f_{n}(Z)$ of $f(Z)$ such that $f_{n}(Z)$ is of standard type 1 or 2 relative to $\left(R_{n}, x_{n}, y_{n}\right)$ if $\nu\left(y_{n-1}\right) \geq \nu\left(x_{n-1}\right)$ and $f_{n}(Z)$ is of standard type 1 relative to $\left(R_{n}, x_{n}, y_{n}\right)$ if $\nu\left(y_{n-1}\right)<\nu\left(x_{n-1}\right)$.

This follows from Theorem 5.3. It is proved in detail in Theorem 4.17 [5].

Lemma 5.6. Suppose that $f(Z) \in K[Z]$. Let $z$ be a root of $f(Z)=0$ in an extension field of $K$ and let $L=K(z)$. Suppose that $\nu$ does not split in $L$. Then we have the following

1. If $f(Z)$ is of standard type 2 relative to $(R, x, y)$ then $[L: K]=p$ and $\operatorname{ord}_{R_{x R}}$ is totally ramified in $L$.

2. If $f(Z)$ is of standard type 1 relative to $(R, x, y)$, then $[L: K]=p$, $\operatorname{ord}_{R_{x R}}$ is totally ramified in $L$, and ord $R_{R_{R}}$ does not split in $L$.

This is proven in Lemma 4.22 [5].

TheOrem 5.7. Suppose that $L$ is a p-extension of $K$ such that $\nu$ does not split in $L$. Then there exists a nonnegative integer $m$ such that for all $n \geq m$, $\operatorname{ord}_{R_{n}}$ does not split in $L$ and $\operatorname{ord}_{\left(R_{n}\right)_{x_{n} R_{n}}}$ is totally ramified in $L$.

Proof. First suppose that $L$ is a separable $p$-cylic extension. Then there exists, as shown in Section $4, z \in L$ and $f(Z) \in R[Z]$ such that $L=K[z], f(Z)$ is the minimal polynomial of $z$ over $K$, and $f(Z)=Z^{p}+G^{p-1} Z+F$ for some $F, G \in R$. Then the conclusions of the theorem follow from Lemmas 5.5 and 5.6, along with the observation that for $n>0$ we have that $\operatorname{ord}_{R_{n-1}}=\operatorname{ord}_{\left(R_{n}\right)_{x_{n} R_{n}}}$ if $x_{n-1}=x_{n}$, $y_{n-1}=x_{n}\left(y_{n}+\alpha_{n}\right)$ and $\operatorname{ord}_{R_{n-1}}=\operatorname{ord}_{\left(R_{n}\right)_{y_{n} R_{n}}}$ if $x_{n-1}=x_{n} y_{n}, y_{n-1}=y_{n}$.

Now suppose that $L$ is of arbitrary degree. Let $H$ be the set of all subfields of $L$ which are separable $p$-cylic extensions of $K$. We have that $L$ is a purely inseparable extension of a finite Galois extension $M$ of $K$. All $K^{\prime} \in H$ are subfields of $M$, and there are only finitely many subfields of $M$ containing $K$. Thus $H$ is a finite set. Further, for $K^{\prime} \in H, \nu$ does not split in $K^{\prime}$. For each $K^{\prime} \in H$ there exists a nonnegative integer $m\left(K^{\prime}\right)$ such that for all $n \geq m\left(K^{\prime}\right)$, ord $R_{n}$ does not split in $K^{\prime}$ and $\operatorname{ord}_{\left(R_{n}\right)_{x_{n} R_{n}}}$ is totally ramified in $K^{\prime}$. Let $m$ be the maximum of the $m\left(K^{\prime}\right)$ for $K^{\prime} \in H$. By Lemma 3.7 it follows that for all $n \geq m$, $\operatorname{ord}_{R_{n}}$ does not split in $L$ and $\operatorname{ord}_{\left(R_{n}\right)_{x_{n} R_{n}}}$ is totally ramified in $L$.

6. On Polynomials. Let $\bar{k}$ be a field of characteristic $p>0$. Let $m=p^{n}$ where $n$ is a nonnegative integer. Let

$$
A(Z)=A_{0}+A_{1} Z+\cdots+A_{e} Z^{e}
$$


where $e$ is a nonnegative integer, $A_{0}, \ldots, A_{e}$ are elements of $\bar{k}$ and $A_{e} \neq 0$. Let $b$ be a nonnegative integer, let $0 \neq D \in \bar{k}$ and let $E_{j}$ be the elements in $\bar{k}$ such that

$$
(D+Z)^{b} A(Z)=\sum_{j} E_{j} Z^{j} .
$$

REMARK 6.1. $E_{j} \neq 0$ for some $j \leq e$.

To see this, consider the smallest $i$ such that $A_{i} \neq 0$.

Lemma 6.2. Assume that $b+e \not \equiv 0(m)$ and $b \equiv 0(m)$. Then there exists $j$ such that $E_{j} \neq 0, j \neq \equiv(m)$ and $j \leq e$.

This is Lemma $6.2[5]$.

Lemma 6.3. Assume that $b+e \not \equiv 0(m)$ and $e<\frac{m}{p}$. Then there exists $j$ such that $E_{j} \neq 0, j \neq 0(m)$ and $j \leq \frac{m}{p}$.

This is Lemma $6.5[5]$

Lemma 6.4. Assume that $b+e \not \equiv 0(m)$ and $e+\frac{m}{p}<m$. Then there exists $j$ such that $E_{j} \neq 0, j \neq 0(m)$ and $j \leq e+\frac{m}{p}$.

This is Lemma $6.7[5]$

7. The Degree $p^{n}$ Case. In this section, we suppose that $R$ is an excellent regular local ring of dimension two, containing an algebraically closed field $\bar{k}$ of characteristic $p>0$ which is isomorphic to the residue field of $R$. Let $K$ be the quotient field of $R$, and let $\nu$ be a real nondiscrete (hence 0 -dimensional) valuation of $K$ which dominates $R$. Let

$$
R \rightarrow R_{1} \rightarrow R_{2} \rightarrow \cdots \rightarrow R_{n} \rightarrow \cdots
$$

be the sequence of quadratic transforms along $\nu$. Recall that $R_{i}$ has "canonical parameters" $x_{i}, y_{i}$, which are defined inductively by $x_{i-1}=x_{i}, y_{i-1}=x_{i}\left(y_{i}+\alpha_{i}\right)$ with $\alpha_{i} \in \bar{k}$ or by $x_{i-1}=x_{i} y_{i}, y_{i-1}=y_{i}$.

Suppose that $f(Z) \in R[Z]$ is monic of degree $m=p^{n} \cdot f(Z)$ has an expansion

$$
f(Z)=Z^{m}+f_{1} Z^{m-1}+\cdots+f_{m-1} Z+F
$$

with $f_{i} \in R$ for $1 \leq i \leq m-1$ and $F \in R$.

DEFINITION 7.1. $f(Z)$ is $R_{i}$-permissible if for all $i \geq 0, f(Z)$ is of nonsplitting type relative to ord $R_{R_{i}}$ and $f(Z)$ is of ramified type relative to ord $\left(R_{i}\right)_{x_{i} R_{i}}$. In particular, for any $R$-transform $f^{\prime}(Z)$ of $f(Z)$, we have

$$
\operatorname{ord}_{R_{i}} f_{q}^{\prime} \geq \frac{q}{m} \operatorname{ord}_{R_{i}} F^{\prime}
$$

and

$$
\operatorname{ord}_{\left(R_{i}\right)_{x_{i} R_{i}}} f_{q}^{\prime}>\frac{q}{m} \operatorname{ord}_{\left(R_{i}\right)_{x_{i} R_{i}}} F^{\prime}
$$

for $0<q<m$.

TheOREM 7.2. Suppose that $L$ is a p-extension of $K$ such that $\nu$ does not split in $L$. Let $z \in L$ be such that $z \notin K$ and $z$ is integral over $R$. Let $m=[K(z): K]$ 
and let $f(Z)$ be the minimal polynomial of $z$ over $K$. Then there exists a nonnegative integer e such that $f(Z)$ is $R_{j}$-permissible for all $j \geq e$.

Proof. In the essential case that $\nu$ is rational nondiscrete, the result follows from Lemmas 3.4 and 3.5 and Theorem 5.7.

Suppose that $F \in R . F$ has a unique series expansion $F=\sum F_{i j} x^{i} y^{j} \in \bar{k}[[x, y]]=$ $\hat{R}$ with $F_{i j} \in \bar{k}$ for all $i, j$. We will also use the following notation. If $f^{\prime}(Z)$ is an $R$-translation of $f(Z)$, then we have a unique expression $f^{\prime}(Z)=Z^{m}+f_{1}^{\prime} Z^{m-1}+$ $\cdots+f_{m-1}^{\prime} Z+F^{\prime}$ with $f_{1}^{\prime}, \ldots, f_{m-1}^{\prime}, F^{\prime} \in R$, and $F^{\prime}=\sum F_{i j}^{\prime} x_{1}^{i} y_{1}^{j}$ with $F_{i j}^{\prime} \in \bar{k}$.

Definition 7.3. Suppose $a, b, c \in \mathbb{N}$. We say that $f(Z)$ is of $R_{i}$-type $(a, b, c)$ if

1. $f(Z)$ is $R_{i}$-permissible

2. $x_{i}^{a} y_{i}^{b}$ divides $F$ in $R_{i}$

3. $\operatorname{ord}_{\left(R_{i}\right)_{y_{i} R_{i}}}\left(f_{q}\right) \geq b \frac{q}{m}$ for $0<q<m$

4. $F_{a, b+c} \neq 0$ and $(a, b+c) \not \equiv 0(m)$.

To $f(Z)$ of $R_{i}$-type $(a, b, c)$ we can associate an integer $[b, c]$ defined by

$$
[b, c]=\left\{\begin{array}{ll}
0 & \text { if } b \equiv 0(m) \text { and } c \leq \frac{m}{p} \\
0 & \text { if } b \neq \equiv(m) \text { and } c<\frac{m}{p} \\
c & \text { if } b \equiv 0(m) \text { and } c>\frac{m}{p} \\
c+\frac{m}{p} & \text { if } b \not \equiv 0(m) \text { and } c \geq \frac{m}{p}
\end{array} .\right.
$$

Theorem 7.7 will tell us that for large $i$, we obtain (after suitable translation) stable forms of $F$, which have $R_{i}$-type $\left(a_{i}, b_{i}, c_{i}\right)$ with $\left[b_{i}, c_{i}\right]=0$. The reason for the complicated definition of $[b, c]$ is that $c$ can in fact go up under quadratic transforms of the type $x=x_{1}, y=x_{1}\left(y_{1}+\alpha\right)$ with $\alpha \neq 0$, when we start with $b>0$, as can be seen from simple examples. A critical point in the proof is that this problem does not occur when $b \equiv 0(m)$.

Definition 7.4. Suppose that $a, b, d \in \mathbb{N}$. We say that $f(Z)$ is of $R_{i}$-antitype $(b, a, d)$ if

1. $f(Z)$ is $R_{i}$-permissible

2. $x_{i}^{a} y_{i}^{b}$ divides $F$ in $R_{i}$

3. $f(Z)$ is of nonsplitting type relative to $\operatorname{ord}_{\left(R_{i}\right)_{y_{i} R_{i}}}$

4. $F_{a+d, b} \neq 0$ and $(a+d, b) \not \equiv 0(m)$.

5. $F_{i j}=0$ whenever $j=b$ and $(i, j) \equiv 0(m)$.

REMARK 7.5. The following observations will be useful.

1. Condition 3 of the definition of $R_{i}$-type is vacuous if $b=0$.

2. If $x=x_{1}$ and $y=x_{1} y_{1}$ then $\operatorname{ord}_{\left(R_{1}\right)_{y_{1} R_{1}}}=\operatorname{ord}_{R_{y R}}$.

Definition 7.6. $f(Z)$ is of R-stable-type $(a, b, c)$ if $f(Z)$ is of $R$-type $(a, b, c)$ with $[b, c]=0$ and $\operatorname{ord}_{R /(x)} \frac{F}{x^{a} y^{b}}=c$. We will say that $f(Z)$ is of $R$-stable-type if $f(Z)$ is of $R$-stable-type $(a, b, c)$ for some $(a, b, c)$.

We may now state the main result of this section.

THEOREM 7.7. Suppose that $f(Z) \in R[Z]$ is irreducible in $K[Z]$ and $f(Z)$ is $R$-permissible. Then there exists $n_{0}$ such that $i \geq n_{0}$ implies that there exists an $R_{i}$-translate $f_{i}(Z)$ of $f(Z)$ such that $f_{i}(Z)$ is of $R_{i}$-stable-type $\left(a_{i}, b_{i}, c_{i}\right)$ for some $\left(a_{i}, b_{i}, c_{i}\right)$, with $b_{i}=0$ if $\nu\left(x_{i-1}\right)=\nu\left(y_{i-1}\right)$. 
We immediately deduce from Theorems 7.2 and 7.7 the following theorem, which will be used in our proof of Local Uniformization (Theorem 9.7 and Corollary 9.8). Since $z$ is integral over $R$, the coefficients of $f(Z)$ are in $R$ (Chapter V, Section 3, Theorem 4 [47]).

THEOREM 7.8. Let $L$ be a p-extension of $K$, and suppose that $\nu$ does not split in $L$. Suppose that $z \in L$ is such that $z \notin K$ and $z$ is integral over $R$. Let $m=[K(z): K]$ and let $f(Z) \in R[Z]$ be the minimal polynomial of $z$ over $K$. Then there exists $n_{0}$ such that $i \geq n_{0}$ implies that there exists an $R_{i}$-translate $f_{i}(Z)$ of $f(Z)$ such that $f_{i}(Z)$ is of $R_{i}$-stable-type $\left(a_{i}, b_{i}, c_{i}\right)$ for some $\left(a_{i}, b_{i}, c_{i}\right)$, with $b_{i}=0$ if $\nu\left(x_{i-1}\right)=\nu\left(y_{i-1}\right)$.

In order to prove Theorem 7.7, we must understand how type and $[b, c]$ transform and antitype and $d$ transform under appropriate translation, followed by a quadratic transform.

LEMmA 7.9. Suppose that $R$ is excellent, $f(Z) \in R[Z]$ is monic irreducible of degree $m>1$ and that $R$ does not split in $K[Z] / f(Z)$. Then $f(Z)$ is irreducible in $\hat{R}[Z]$ where $\hat{R}$ is the completion of $R$ with respect to $M(R)$. In particular, $f(\bar{z}) \neq 0$ for all $\bar{z} \in \hat{R}$.

Proof. Let $\bar{z}$ be the class of $Z$ in $K[Z] /(f(Z))$, so that $K[Z] /(f(Z))=K[\bar{z}]$. Let $S$ be the integral closure of $R$ in $K[\bar{z}] . S$ is a finite $R$-module since $R$ is excellent. $S$ is quasilocal by assumption, so that $S$ is an excellent local ring. $S$ is normal of dimension two, so that $S$ is Cohen Macaulay. Thus $S$ is a free $R$-module, of some finite rank $m$ (c.f. Theorem 46 [38]). Since $S$ is local, and by Theorem 55 [38], $S \otimes_{R} \hat{R} \cong \hat{S}$, where $\hat{R}$ denotes the completion of $R$ with respect to its maximal ideal, and $\hat{S}$ denotes the completion of $S$ with respect to its maximal ideal. Thus $\hat{S}$ is a free $\hat{R}$ module of rank $m$. Since $S$ is normal and excellent, $\hat{S}$ is normal, and is thus a domain. Now we have that $1, \bar{z}, \ldots, \bar{z}^{d-1}$ (where $d=\operatorname{deg} f(Z)$ ) generate a free $R$ submodule of $S$, so they must generate a free $\hat{R}$ submodule of $\hat{S}$ of rank $d$ (as completion is flat). Suppose $f(Z)$ factors as $f(Z)=f_{1}(Z) f_{2}(Z)$ with $f_{1}(Z), f_{2}(Z) \in \hat{R}[Z]$, and $0<\operatorname{deg} f_{1}(Z)$, $0<\operatorname{deg} f_{2}(Z)$. Then $0=f(\bar{z})=f_{1}(\bar{z}) f_{2}(\bar{z})$ which implies that $f_{1}(\bar{z})=0$ or $f_{2}(\bar{z})=0$ since $\hat{S}$ is a domain, which is a contradiction, since $\operatorname{deg} f_{1}(Z)<d$ and $\operatorname{deg} f_{2}(Z)<d$.

\section{口}

Lemma 7.10. Suppose that $R$ is excellent and $f(Z) \in R[Z]$ is an irreducible monic polynomial of degree $m=p^{n}>1$. Assume that $f(Z)$ is of ramified type relative to ord $R$ and that $R$ does not split in $K[Z] /(f(Z))$. Then there exists an $R$ translate $f^{\prime}(Z)$ of $f(Z)$ such that $f^{\prime}(0) \neq 0$ and $F_{i j}^{\prime}=0$ whenever $(i, j) \equiv 0(m)$ and $i+j \leq \operatorname{ord}_{R} f^{\prime}(0)$.

Proof. Let $\hat{R}$ be the completion of $R$ with respect to its maximal ideal. By Lemma $7.9, f(\bar{z}) \neq 0$ for all $\bar{z} \in \hat{R}$. Now the desired conclusions follow from Lemma 8.14 [5]. (

LEMMA 7.11. Suppose that $x=x_{1}$ and $y=x_{1}\left(y_{1}+\alpha_{1}\right)$ with $\alpha_{1} \in \bar{k}$. Suppose that $f(Z)$ is of $R$-type $(a, b, c)$. Then there exists an $R$-translate $f^{\prime}(Z)$ of $f(Z)$ such that $f^{\prime}(Z)$ is of $R$-type $(a, b, c)$ and if $d=$ ord $F^{\prime}, i+j=d$ and $(i, j) \equiv 0(m)$, then $F_{i, j}^{\prime}=0$.

This is Lemma 9.2 [5]. The essential point is that $f(Z)$ is of ramified type relative to $\operatorname{ord}_{R}=\operatorname{ord}_{\left(R_{1}\right)_{x_{1} R_{1}}}$. 
Lemma 7.12. Suppose that $f(Z)$ is of $R$-type $(a, b, c)$. Then there exists an $R$ translate $f^{\prime}(Z)$ of $f(Z)$ such that $f^{\prime}(Z)$ is of $R$-type $(a, b, c)$ and if $(a, b) \equiv 0(m)$, then $F_{a, b}^{\prime}=0$.

This is Lemma $9.3[5]$.

Lemma 7.13. Suppose that $f(Z)$ is of $R$-type $(a, b, c)$ with $c<m$. Then there exists an $R$-translate $f^{\prime}(Z)$ of $f(Z)$ such that $f^{\prime}(Z)$ is of $R$-type $(a, b, c)$ and if $d=$ $\operatorname{ord}_{R} F^{\prime}, i+j=d$ and $(i, j) \equiv 0(m)$, then $F_{i, j}^{\prime}=0$.

This is Lemma 9.4 [5].

Lemma 7.14. Suppose that $f(Z)$ is of $R$-antitype $(b, a, c)$ where $c<m$. Then there exists an $R$-translate $f^{\prime}(Z)$ of $f(Z)$ such that $f^{\prime}(Z)$ is of $R$-antitype $(b, a, c)$ and if $d=\operatorname{ord} F^{\prime}, i+j=d$ and $(i, j) \equiv 0(m)$, then $F_{i, j}^{\prime}=0$.

This is Lemma 9.5 [5].

Lemma 7.15. Suppose that $f(Z)$ is of $R$-type $(a, b, c)$ where $[b, c]=0$. Then there exists an $R$-translate $f^{\prime}(Z)$ of $f(Z)$ such that $f^{\prime}(Z)$ is of $R$-stable-type $\left(a, b, c^{\prime}\right)$ where $c^{\prime} \leq c$.

This is Lemma 9.6 [5].

Lemma 7.16. Suppose that $f(Z)$ is of $R$-stable-type $(a, b, c)$. Then for all $i \geq 1$ there exists an $R_{i}$-translate $f_{i}(Z)$ of $f(Z)$ such that $f_{i}(Z)$ is of $R_{i}$-stable-type $\left(a_{i}, b_{i}, c_{i}\right)$ for some $\left(a_{i}, b_{i}, c_{i}\right)$ with $b_{i}=0$ if $\nu\left(x_{i-1}\right)=\nu\left(y_{i-1}\right)$.

This is Lemma 9.9 [5].

Lemma 7.17. Suppose that $x=x_{1}$ and $y=x_{1}\left(y_{1}+\alpha_{1}\right)$ with $\alpha_{1} \in \bar{k}$. Suppose that $f(Z)$ is of R-type $(a, b, c)$. Further suppose $b \equiv 0(m)$ or $[b, c]<m$. Then there exists an $R$-translate $f_{1}(Z)$ of $f(Z)$ such that $f_{1}(Z)$ is of $R_{1}$-type $\left(a_{1}, b_{1}, c_{1}\right)$ with $\left[b_{1}, c_{1}\right] \leq[b, c]$, and $b_{1}=b$ if $\alpha_{1}=0$ and $b_{1}=0$ if $\alpha_{1} \neq 0$.

Proof. By Lemma 7.11, after making an $R$-translate, we may assume that the coefficents $F_{i j}$ of $x^{i} y^{j}$ in the expansion of $F$ are zero whenever $i+j=a_{1}$ and $(i, j) \equiv$ $0(m)$, where $a_{1}=\operatorname{ord}_{R}(F)$. Let $q$ be the largest integer such that there exists $i$ with $i+q=a_{1}$ and $F_{a_{1}-q, q} \neq 0$. We have

$$
q-b \leq a_{1}-a-b \leq c .
$$

We first prove the lemma in the case when $\alpha_{1}=0$. We have an expression

$$
F=x_{1}^{a_{1}} y_{1}^{b}\left(\sum_{j=0}^{q-b} F_{a_{1}-j-b, j+b} y_{1}^{j}+x_{1} \Omega\right)
$$

with $\Omega \in R_{1}$. Let $c_{1}=q-b$. Then (23) implies $c_{1} \leq c$. Since $\left(a_{1}-q, q\right) \not \equiv 0(m)$, we have that $\left(a_{1}, b+c_{1}\right) \not \equiv 0(m)$. Thus by Remark $7.5, f(Z)$ is of $R_{1}$-type $\left(a_{1}, b, c_{1}\right)$. We have that $\left[b, c_{1}\right] \leq[b, c]$.

Now assume that $\alpha_{1} \neq 0$. We have an expression

$$
F=x_{1}^{a_{1}}\left(y_{1}+\alpha_{1}\right)^{b}\left[\sum_{j=0}^{q-b} F_{a_{1}-j-b, j+b}\left(y_{1}+\alpha_{1}\right)^{j}+x_{1} \Omega\right]
$$


with $\Omega \in R_{1}$. Expand

$$
\left(y_{1}+\alpha_{1}\right)^{b} \sum_{j=0}^{q-b} F_{a_{1}-j-b, j+b}\left(y_{1}+\alpha_{1}\right)^{j}=\sum_{i=0}^{q} E_{i} y_{1}^{i}
$$

with $E_{i} \in \bar{k}$. By remark 6.1, this polynomial has a nonzero $E_{i}$ term with $i \leq q-b$. Thus if $a_{1} \not \equiv 0(m)$, we have by Remark 7.5 that $f(Z)$ is of $R_{1}$-type $\left(a_{1}, 0, i\right)$ with $i \leq q-b \leq c$, and $[0, i] \leq[b, c]$.

Assume that $a_{1} \equiv 0(m)$, so that $q \not \equiv 0(m)$. If $b \equiv 0(m)$ then Lemma 6.2 implies that $E_{i} \neq 0$ for some $i$ with $i \neq \equiv(m)$ and $i \leq q-b$. We then have by Remark 7.5 that $F$ is of $R_{1}$-type $\left(a_{1}, 0, i\right)$ with $[0, i] \leq[b, c]$.

We have two remaining cases. First assume that $a_{1} \equiv 0(m), b \neq \equiv(m)$ and $[b, c]<\frac{m}{p}$. Then $c<\frac{m}{p}$ and (23) implies $q-b<\frac{m}{p}$. Since $q \not \equiv 0(m)$, Lemma 6.3 implies that $E_{i} \neq 0$ for some $i$ with $i \not \equiv 0(m)$ and $i \leq \frac{m}{p}$. Thus by Remark 7.5, $f(Z)$ is of $R_{1}$-type $\left(a_{1}, 0, i\right)$ with $[0, i]=0$. $m$ and

Now assume that $a_{1} \equiv 0(m), b \not \equiv 0(m)$ and $\frac{m}{p} \leq[b, c]<m$. Then $[b, c]=c+\frac{m}{p}<$

$$
q-b+\frac{m}{p} \leq c+\frac{m}{p}<m
$$

by (23). Since $q \not \equiv 0(m)$, Lemma 6.4 implies $E_{i} \neq 0$ for some $i$ with $i \not \equiv 0(m)$ and $i \leq q-b+\frac{m}{p} \leq c+\frac{m}{p}$. Thus by Remark 7.5, $f(Z)$ is of $R_{1}$-type $\left(a_{1}, 0, i\right)$ with $[0, i]=i \leq c+\frac{m}{p}=[b, c]$.

Lemma 7.18. Suppose that $x=x_{1}$ and $y=x_{1}\left(y_{1}+\alpha_{1}\right)$ with $\alpha_{1} \in \bar{k}$. Suppose that $f(Z)$ is of $R$-antitype $(b, a, d)$. Then there exists an $R$-translate $f_{1}(Z)$ of $f(Z)$ such that:

1. Suppose $d<m$ and $a \equiv 0(m)$. Then one of the following holds:

a. $f_{1}(Z)$ is of $R_{1}$-antitype $\left(b_{1}, a_{1}, d_{1}\right)$ with $d_{1}<d$ and $a_{1} \equiv 0(m)$, or

b. $f_{1}(Z)$ is of $R_{1}$-type $\left(a_{1}, b_{1}, c_{1}\right)$ with $b_{1} \equiv 0(m)$ and $\left[b_{1}, c_{1}\right]<m$.

2. Suppose $d \leq \frac{m}{p}$ and either $d<\frac{m}{p}$ or $a+b+\frac{m}{p} \not \equiv 0(m)$. Then $f_{1}(Z)$ is of $R_{1}$-type $\left(a_{1}, b_{1}, c_{1}\right)$ with $\left[b_{1}, c_{1}\right]=0$ where $b_{1}=b$ if $\alpha_{1}=0$ and $b_{1}=0$ if $\alpha_{1} \neq 0$.

Proof. By Lemma 7.14, after making an $R$-translate, we may assume that the coefficents $F_{i j}$ of $x^{i} y^{j}$ in the expansion of $F$ are zero whenever $i+j=a_{1}$ and $(i, j) \equiv$ $0(m)$, where $a_{1}=\operatorname{ord}_{R}(F)$. Let $q$ be the largest integer such that there exists $i$ with $i+q=a_{1}$ and $F_{a_{1}-q, q} \neq 0$. We have

$$
q-b \leq a_{1}-a-b \leq d .
$$

Case 1. Assume $\alpha_{1}=0$.

We will first establish 1 of the conclusions of the lemma. We are assuming that $d<m$ and $a \equiv 0(m)$. Set $b=b^{\prime}+r$ where $b^{\prime} \equiv 0(m)$ and $0 \leq r<m$.

First assume that $a_{1}-a-b^{\prime}<m$. Then we have an expression

$$
F=x_{1}^{a_{1}} y_{1}^{b^{\prime}}\left(\sum_{j=r}^{q-b^{\prime}} F_{a_{1}-j-b^{\prime}, j+b^{\prime}} y_{1}^{j}+x_{1} \Omega\right)
$$


with $\Omega \in R_{1}$. Let $c_{1}=q-b^{\prime}$. We have $c_{1} \leq a_{1}-a-b^{\prime}<m$. We have $c_{1}>0$, since if $c_{1}=0$, we have $b=b^{\prime}$, and combining this with the fact that $a \equiv 0(m)$, we obtain $(a, b) \equiv 0(m)$, which is a contradiction.

Thus we have $\left(a_{1}, b^{\prime}+c_{1}\right) \not \equiv 0(m)$, and by Remark $7.5, f_{1}(Z)$ is of $R_{1}$-type $\left(a_{1}, b^{\prime}, c_{1}\right)$ with $b^{\prime} \equiv 0(m)$ and $c_{1}<m$.

Now assume that $a_{1}-a-b^{\prime} \geq m$. Then $d>0$ by (24) and since $r=b-b^{\prime}<m$. Let $a_{1}=a^{\prime}+s$ where $a^{\prime} \equiv 0(m)$ and $0 \leq s<m$. Let $d_{1}=a+d+b-a^{\prime}$. We have $x^{a+d} y^{b}=x_{1}^{a^{\prime}} y_{1}^{b} x_{1}^{d_{1}}$ so

$$
F=x_{1}^{a^{\prime}} y_{1}^{b} \Lambda
$$

where $\Lambda \in \hat{R}_{1}$ has a nonzero $x_{1}^{d^{\prime}}$ term. We have $a+b^{\prime} \leq a_{1}-m=\left(a^{\prime}-m\right)+s$. Since $a+b^{\prime} \equiv 0(m)$ and $\left(a^{\prime}-m\right) \equiv 0(m)$ we have that $a+b^{\prime} \leq a^{\prime}-m$, so $0<m-r \leq a_{1}-a-b$, which implies $d_{1}<d$. By (24), and the assumption $d<m$, we have $a_{1}-a-b<m$. Since we are also assuming $a_{1}-a-b^{\prime} \geq m$, we have $b \neq b^{\prime}$. Thus by Remark 7.5, $f_{1}(Z)$ is of $R_{1}$-antitype $\left(b, a^{\prime}, d_{1}\right)$, with $a^{\prime} \equiv 0(m)$. We have thus established 1 of the lemma in the case that $\alpha_{1}=0$.

We will now establish 2 of the conclusions of the lemma, with the assumption that $\alpha_{1}=0$. We have a form

$$
F=x_{1}^{a_{1}} y_{1}^{b}\left(\sum_{j=0}^{q-b} F_{a_{1}-j-b, j+b} y_{1}^{j}+x_{1} \Omega\right)
$$

with $\Omega \in R_{1}, F_{a_{1}-q, q} \neq 0$ and $\left(a_{1}, q\right) \not \equiv 0(m)$. If $q-b<\frac{m}{p}$, then set $c_{1}=q-b$. We have that $f(Z)$ is of $R_{1}$-type $\left(a_{1}, b, c_{1}\right)$ with $\left[b, c_{1}\right]=0$ as desired.

Suppose that $q-b \geq \frac{m}{p}$. By (24) and the assumption $d \leq \frac{m}{p}$, we conclude that $q-b=a_{1}-a-b=d=\frac{m}{p}$. By our assumptions, we must have that $a+b+\frac{m}{p} \not \equiv$ $0(m)$. Thus $a_{1} \not \equiv 0(m)$. We have $F_{a_{1}-b, b}=F_{a+d, b} \neq 0$ and $x^{a+d} y^{b}=x_{1}^{a_{1}} y_{1}^{b}$. Thus $F=x_{1}^{a_{1}} y_{1}^{b} \Lambda$ where $\Lambda \in R_{1}$ is a unit. Since $\left(a_{1}, b\right) \not \equiv 0(m)$, we have that $f(Z)$ is of $R_{1}$-type $\left(a_{1}, b, 0\right)$, with $[b, 0]=0$.

Case 2. Assume $\alpha_{1} \neq 0$.

We will first establish 1 of the conclusions of the lemma. Our assumptions are that $d<m$ and $a \equiv 0(m)$. We have an expression

$$
F=x_{1}^{a_{1}}\left(y_{1}+\alpha_{1}\right)^{b}\left[\sum_{j=0}^{q-b} F_{a_{1}-j-b, j+b}\left(y_{1}+\alpha_{1}\right)^{j}+x_{1} \Omega\right]
$$

with $\Omega \in R_{1}$. Expand

$$
\left(y_{1}+\alpha_{1}\right)^{b} \sum_{j=0}^{q-b} F_{a_{1}-j-b, j+b}\left(y_{1}+\alpha_{1}\right)^{j}=\sum_{i=0}^{q} E_{i} y_{1}^{i}
$$

with $E_{i} \in \bar{k}$. This polynomial has a nonzero $E_{i}$ term with $i \leq q-b$ by Remark 6.1. Thus if $a_{1} \not \equiv 0(m)$, we have by Remark 7.5 that $f(Z)$ is of $R_{1}$-type $\left(a_{1}, 0, i\right)$ with $[0, i]=i \leq q-b \leq d<m$.

Assume that $a_{1} \equiv 0(m)$. Then $q \not \equiv 0(m)$. If $b \equiv 0(m)$ then Lemma 6.2 implies that $E_{i} \neq 0$ for some $i$ with $i \not \equiv 0(m)$ and $i \leq q-b$. We then have that $F$ is of $R_{1}$-type $\left(a_{1}, 0, i\right)$ with $[0, i]=i \leq d<m$. 
Now assume that we are in the remaining case $a_{1} \equiv 0(m)$ and $b \not \equiv 0(m)$. Write $b=b^{\prime}+r$ with $b^{\prime} \equiv 0(m)$ and $0 \leq r<m$. We then have an expression

$$
F=x_{1}^{a_{1}}\left(y_{1}+\alpha_{1}\right)^{b^{\prime}}\left[\sum_{j=r}^{q-b^{\prime}} F_{a_{1}-j-b^{\prime}, j+b^{\prime}}\left(y_{1}+\alpha_{1}\right)^{j}+x_{1} \Omega\right]
$$

with $\Omega \in R_{1}$. Expand

$$
\left(y_{1}+\alpha_{1}\right)^{b^{\prime}} \sum_{j=r}^{q-b^{\prime}} F_{a_{1}-j-b^{\prime}, j+b^{\prime}}\left(y_{1}+\alpha_{1}\right)^{j}=\sum_{i=0}^{q} D_{i} y_{1}^{i}
$$

with $D_{i} \in \bar{k}$. We have $q \not \equiv 0(m)$ and $b^{\prime} \equiv 0(m)$. Thus Lemma 6.2 implies $D_{i} \neq 0$ for some $i$ with $i \not \equiv 0(m)$ and $i \leq q-b^{\prime} \leq a_{1}-a-b^{\prime}$. Let $c_{1}=i$. $f_{1}(Z)$ is of $R_{1}$-type $\left(a_{1}, 0, c_{1}\right)$. From $0<b-b^{\prime}<m, 0 \leq a_{1}-a-b<m$ and $\left(b-b^{\prime}\right)+\left(a_{1}-a-b\right)=$ $a_{1}-a-b^{\prime} \equiv 0(m)$, we infer that $a_{1}-a-b^{\prime}=m$. Thus $c_{1}<m$. We have thus established 1 of the conclusions of the lemma in the case when $\alpha_{1} \neq 0$.

We will now establish 2 of the conclusions of the lemma, with the assumption that $\alpha_{1} \neq 0$. We have a form

$$
F=x_{1}^{a_{1}}\left(y_{1}+\alpha\right)^{b}\left[\sum_{j=0}^{q-b} F_{a_{1}-j-b, j+b}\left(y_{1}+\alpha_{1}\right)^{j}+x_{1} \Omega\right]
$$

with $\Omega \in R_{1}$ and $F_{a_{1}-q, q} \neq 0$. Expand

$$
\left(y_{1}+\alpha_{1}\right)^{b} \sum_{j=0}^{q-b} F_{a_{1}-j-b, j+b}\left(y_{1}+\alpha_{1}\right)^{j}=\sum_{i=0}^{q} E_{i} y_{1}^{i}
$$

with $E_{i} \in \bar{k}$.

First suppose $q-b<\frac{m}{p}$. If $a_{1} \not \equiv 0(m)$, then the polynomial (25) has a nonzero $E_{i}$ term with $i \leq q-b$, by Remark 6.1 , and we have that $f(Z)$ is of $R_{1}$-type $\left(a_{1}, 0, i\right)$ with $[0, i]=0$ as desired.

If $q-b<\frac{m}{p}$ and $a_{1} \equiv 0(m)$, then $q \not \equiv 0(m)$. By Lemma 6.3, there exists $j$ such that $E_{j} \neq 0, j \not \equiv 0(m)$ and $j \leq \frac{m}{p}$. Thus $f(Z)$ is of $R_{1}$-type $(a, 0, i)$ with $[0, i]=0$.

Suppose that $q-b \geq \frac{m}{p}$. By (24), and since $d \leq \frac{m}{p}$, we have $q-b=a_{1}-a-b=$ $d=\frac{m}{p}$. By our assumptions, we must have that $a+b+\frac{m}{p} \not \equiv 0(m)$. Thus $a_{1} \not \equiv 0(m)$. We have $F_{a_{1}-b, b}=F_{a+d, b} \neq 0$. Since $a_{1} \neq \equiv(m)$, we have that $f(Z)$ is of $R_{1}$-type $\left(a_{1}, 0,0\right)$, with $[0,0]=0$.

Lemma 7.19. Suppose that $x=x_{1} y_{1}$ and $y=y_{1}$. Suppose that $f(Z)$ is of $R$-type $(a, b, c)$. Then there exists an $R$-translate $f_{1}(Z)$ of $f(Z)$ such that:

1. Suppose $b \equiv 0(m)$. Then one of the following holds:

a. $f_{1}(Z)$ is of $R_{1}$-type $\left(a, b_{1}, c_{1}\right)$ with $\left[b_{1}, c_{1}\right]<[b, c]$ and $b_{1} \equiv 0(m)$, or

b. $f_{1}(Z)$ is of $R_{1}$-antitype $\left(b_{1}, a_{1}, d_{1}\right)$ with $d_{1}<m$ and $a_{1} \equiv 0(m)$.

2. Suppose $[b, c]<m$. Then one of the following holds:

a) $f_{1}(Z)$ is of $R_{1}$-type $\left(a, b_{1}, c_{1}\right)$ with $\left[b_{1}, c_{1}\right] \leq \max \{0,[b, c]-1\}$.

b) $f_{1}(Z)$ is of $R_{1}$-antitype $\left(b_{1}, a, d_{1}\right)$ with $d_{1}<\frac{m}{p}$.

c) $f_{1}(Z)$ is of $R_{1}$-type $\left(a_{1}, b_{1}, c_{1}\right)$ with $\left[b_{1}, c_{1}\right]=[b, c]$ and of $R_{1}$-antitype $\left(b_{1}, a, d_{1}\right)$ with $d_{1}=\frac{m}{p}$ and $a+b_{1}+\frac{m}{p} \not \equiv 0(m)$. 
Proof. Let $d=\operatorname{ord}_{R}(F)$. Let $d=b_{1}+s$ where $b_{1} \equiv 0(m)$ and $0 \leq s<m$. Let $a=a^{\prime}+r$ with $a^{\prime} \equiv 0(m)$ and $0 \leq r<m$.

Case 1. Assume $b \equiv 0(m)$.

By Lemma 7.12, after making an $R$-translate of $f(Z)$, we may assume that

$$
F_{a, b}=0 \text { if }(a, b) \equiv 0(m) .
$$

Case 1.1. Assume that $b \equiv 0(m)$ and $d-a^{\prime}-b \geq m$.

We have $F=x_{1}^{a} y_{1}^{b_{1}} \Lambda$ with $\Lambda \in R_{1}$. The nonzero $x^{a} y^{b+c}$ term of $F$ transforms to a nonzero term $x_{1}^{a} y_{1}^{b_{1}} y_{1}^{a+b+c-b_{1}}$ in the expansion of $F$ in $x_{1}$ and $y_{1}$. Since $(a, b+c) \not \equiv$ $0(m)$, we have that $F$ is of $R_{1}$-type $\left(a, b_{1}, c_{1}\right)$ with $c_{1}=a+b+c-b_{1}$. From the assumptions $b \equiv 0(m)$ and $d-a^{\prime}-b \geq m$ we will show that $c_{1}<c$.

We have that $b_{1}-a^{\prime}-b \geq m$ since $b_{1}-a^{\prime}-b \equiv 0(m)$ and $\left(b_{1}-a^{\prime}-b\right)+\left(d-b_{1}\right)=$ $d-a^{\prime}-b \geq m$. Further,

$$
b_{1}-a-b=\left(b_{1}-a^{\prime}-b\right)-\left(a-a^{\prime}\right) \geq m-\left(a-a^{\prime}\right)>0 .
$$

Thus $c_{1}=a+b+c-b_{1}<c$. Since $\operatorname{ord}_{R}=\operatorname{ord}_{\left(R_{1}\right)_{y_{1} R_{1}}}$ and $f(Z)$ is $R$-permissible, it follows that $f(Z)$ is of $R_{1}$-type $\left(a, b_{1}, c_{1}\right)$ with $\left[b_{1}, c_{1}\right]<[b, c]$ and $b_{1} \equiv 0(m)$.

Case 1.2. Assume that $b \equiv 0(m)$ and $d-a^{\prime}-b<m$.

We have $F=x_{1}^{a^{\prime}} y_{1}^{d} \Lambda$ with $\Lambda \in R_{1}$. We will show that $F$ has a nonzero $x^{i} y^{j}$ term where $i+j=d$ and $(i, j) \not \equiv 0(m)$. This is the case if $d \not \equiv 0(m)$, so suppose that $d \equiv 0(m)$. Since $d-a^{\prime}-b \equiv d(m)$ and

$$
0 \leq d-a-b \leq d-a^{\prime}-b<m,
$$

we have $d=a+b$. Thus $(a, b) \equiv 0(m)$, a contradiction to (26). In particular, we have $d \not \equiv 0(m)$.

We have that $a \leq i \leq d-b$. The $x^{i} y^{j}$ term transforms to a nonzero $x_{1}^{a^{\prime}} y_{1}^{d} x_{1}^{i-a^{\prime}}$ term in the expansion of $F$ in $x_{1}$ and $y_{1}$. Let $d_{1}=i-a^{\prime}$. We have $i-a^{\prime} \leq d-b-a^{\prime}<m$ by assumption. Since $\operatorname{ord}_{R}=\operatorname{ord}_{y_{1} R_{1}}$ and $f(Z)$ is $R$-permissible, and $d \not \equiv 0(m)$, we have that $f(Z)$ is of $R_{1}$-antitype $\left(d, a^{\prime}, d_{1}\right)$ with $d_{1}<m$ and $a^{\prime} \equiv 0(m)$.

Case 2. Assume that $[b, c]<m$.

By Lemma 7.13, after making an $R$-translate of $f(Z)$, we may assume that $F_{i j}=0$ whenever $i+j=d=\operatorname{ord}_{R} F$ and $(i, j) \equiv 0(m)$.

Case 2.1. Assume that $[b, c]<m$ and $d-a-b \geq \frac{m}{p}$.

This case is like Case 1.1. We have $F=x_{1}^{a} y_{1}^{d} \Lambda$ with $\Lambda \in R_{1}$. The $x^{a} y^{b+c}$ term of $F$ transforms to a nonzero $x_{1}^{a} y_{1}^{d} y_{1}^{a+b+c-d}$ term in the expansion of $F$ in $x_{1}$ and $y_{1}$. Let $c_{1}=a+b+c-d$. We have that $(a, a+b+c) \not \equiv 0(m)$ since $(a, b+c) \not \equiv 0(m)$. 
Thus $f(Z)$ is of $R_{1}$-type $\left(a, d, c_{1}\right)$. From $d-a-b \geq \frac{m}{p}$ we conclude that $c_{1} \leq c-\frac{m}{p}$. In particular, if $d-a-b>\frac{m}{p}$ we have that $\left[d, c_{1}\right]<[b, c]$.

Case 2.2. Assume that $[b, c]<m$ and $d-a-b<\frac{m}{p}$.

This case is like Case 1.2. We have $F=x_{1}^{a} y_{1}^{d} \Lambda$ with $\Lambda \in R_{1}$, and $F$ has a nonzero $x^{i} y^{j}$ term where $i+j=d$ and $(i, j) \not \equiv 0(m)$. We have that $a \leq i \leq d-b$. This terms transforms to a nonzero $x_{1}^{a} y_{1}^{d} x_{1}^{i-a}$ term in the expansion of $F$ in $x_{1}$ and $y_{1}$. Let $d_{1}=i-a$. We have $i-a \leq d-b-a<\frac{m}{p}$ by assumption. Since $\operatorname{ord}_{R}=\operatorname{ord}_{y_{1} R_{1}}$, and $f(Z)$ is $R$-permissible, we have that $f(Z)$ is of $R_{1}$-antitype $\left(d, a, d_{1}\right)$ with $d_{1}<\frac{m}{p}$.

Case 2.3. Assume that $[b, c]<m$ and $d-a-b=\frac{m}{p}$.

If $b \not \equiv 0(m)$ then we have from Case 2.1 that $f(Z)$ is of $R_{1}$-type $\left(a, d, c_{1}\right)$, with $c_{1} \leq c-\frac{m}{p}$, so that $\left[d, c_{1}\right]<[b, c]$. We now analyze Case 2.3 with the additional assumption that $b \equiv 0(m)$. Recall that $a=a^{\prime}+r$ with $a^{\prime} \equiv 0(m)$ and $0 \leq r<m$, and $d=b_{1}+s$ with $b_{1} \equiv 0(m)$ and $0 \leq s<m$.

Assume that $b \equiv 0(m)$ and $d-a^{\prime}-b \geq m$. We have $F=x_{1}^{a} y_{1}^{b_{1}} \Lambda$ with $\Lambda \in R_{1}$. The nonzero term $x^{a} y^{b+c}$ (with $\left.(a, b+c) \not \equiv 0(m)\right)$ in $F$ transforms to a nonzero $x_{1}^{a} y_{1}^{a+b+c}=x_{1}^{a} y_{1}^{b_{1}} y_{1}^{a+b+c-b_{1}}$ term in the expansion of $F$ in $x_{1}$ and $y_{1}$. Let $c_{1}=$ $a+b+c-b_{1}$. We have that $\left(b_{1}-a^{\prime}-b\right)+\left(d-b_{1}\right)=d-a^{\prime}-b \geq m$. Since $b_{1}-a^{\prime}-b \equiv 0(m)$ and $0 \leq d-b_{1}<m$ we conclude that $b_{1}-a^{\prime}-b \geq m$. Now from $b_{1}-a-b=\left(b_{1}-a^{\prime}-b\right)-\left(a-a^{\prime}\right) \geq m-\left(a-a^{\prime}\right)$ we conclude that $b_{1}-a-b>0$. Thus $c_{1}=a+b+c-b_{1}<c$. Since $b \equiv 0$ and $b_{1} \equiv 0$ we have that $f(Z)$ is of $R_{1}$-type $\left(a, b_{1}, c_{1}\right)$ with $\left[b_{1}, c_{1}\right] \leq \max \{0,[b, c]-1\}$.

Assume that $b \equiv 0(m), d-a^{\prime}-b<m, p \neq 2$ (and $d-a-b=\frac{m}{p}$ ). We have $F=x_{1}^{a} y_{1}^{d} \Lambda$ with $\Lambda \in R_{1}$. $F$ has a nonzero $x^{i} y^{j}$ term with $i+j=d$ and $(i, j) \not \equiv 0(m)$. We necessarily have $a \leq i \leq d-b$. Let $d_{1}=i-a$. This term transforms to a nonzero $x_{1}^{a} y_{1}^{d} x_{1}^{d_{1}}$ term in the expansion of $F$ in terms of $x_{1}$ and $y_{1}$. We have $d_{1} \leq d-a-b=\frac{m}{p}$ and $\left(a+d_{1}, d\right) \not \equiv 0(m)$. Since $\operatorname{ord}_{R}=\operatorname{ord}_{y_{1} R_{1}}$, and $f(Z)$ is $R$-permissible, $f(Z)$ is of $R_{1}$-antitype $\left(d, a, d_{1}\right)$ with $d_{1} \leq \frac{m}{p}$. If $d_{1}<\frac{m}{p}$, then we have achieved the conclusions $2 \mathrm{~b})$ of the lemma.

Suppose $d_{1}=\frac{m}{p}$.

$$
a-a^{\prime}+\frac{m}{p}=\left(a-a^{\prime}\right)+(d-a-b)=d-a^{\prime}-b<m .
$$

Since $p \neq 2$, we have that $2\left(a-a^{\prime}+\frac{m}{p}\right) \not \equiv 0(m)$. As $a^{\prime} \equiv 0(m)$, we have $2\left(a+\frac{m}{p}\right) \not \equiv$ $0(m)$. Since $d-a-b=\frac{m}{p}$, we have

$$
a+d+\frac{m}{p}=2\left(a+\frac{m}{p}\right)+b \equiv 2\left(a+\frac{m}{p}\right) \bmod (m),
$$

and thus $a+d+\frac{m}{p} \not \equiv 0(m)$. From this analysis and Case 2.1, we have the conclusions of 2c) of the lemma.

Finally, we consider the case $p=2, b \equiv 0(m)$ and $d-a-b=\frac{m}{p}$. We have $F=x_{1}^{a} y_{1}^{d} \Lambda$ with $\Lambda \in R_{1}$. $F$ has a nonzero $x^{a} y^{b+c}$ term, with $(a, b+c) \not \equiv 0(m)$, which transforms to a nonzero $x_{1}^{a} y_{1}^{d} y_{1}^{c_{1}}$ term with $c_{1}=a+b+c-d$ in the expansion of $F$ in 
terms of $x_{1}$ and $y_{1}$. We have $c_{1}=c-\frac{m}{p}$. Since $[b, c]<m$, we have $c<m$, and thus $c_{1}<\frac{m}{2}$. Thus $f(Z)$ is of $R_{1}$-type $\left(a, d, c_{1}\right)$ with $\left[d, c_{1}\right]=0$.

Lemma 7.20. Suppose that $x=x_{1} y_{1}$ and $y=y_{1}$. Suppose that $f(Z)$ is of $R$ antitype $(b, a, d)$ with $d<m$. Then there exists an $R$-translate $f_{1}(Z)$ of $f(Z)$ such that $f_{1}(Z)$ is of $R_{1}$-antitype $\left(b_{1}, a, d_{1}\right)$ with $d_{1} \leq d$,

Proof. By Lemma 7.14, after making an $R$-translate of $f(Z)$, we may assume that $F_{i, j}=0$ if $i+j=\operatorname{ord}_{R}(F)$ and $(i, j) \equiv 0(m)$. Let $e=\operatorname{ord}_{R}(F)$. We have $F=x_{1}^{a} y_{1}^{e} \Lambda$ with $\Lambda \in R_{1}$. $F$ has an $x^{i} y^{j}$ term with $i+j=e$ and $(i, j) \not \equiv 0(m)$. Let $d_{1}=i-a$. We have $d_{1} \leq e-a-b \leq d$. $F_{1}$ has a nonzero $x_{1}^{a} y_{1}^{e} y_{1}^{d_{1}}$ term in its expansion in $x_{1}$ and $y_{1}$, with $\left(e, a+d_{1}\right) \not \equiv 0(m)$. Since $\operatorname{ord}_{R}=\operatorname{ord}_{\left(R_{1}\right)_{y_{1} R_{1}}}$, and $f(Z)$ is $R$-permissible, $f(Z)$ is of $R_{1}$-antitype $\left(e, a, d_{1}\right)$, with $d_{1} \leq d$.

Now we prove Theorem 7.7 in the essential case when $\nu$ is rational nondiscrete. The proof is in five steps.

Step 1. There exists a sequence of quadratic transforms $R \rightarrow R_{t}$ along $\nu$ and an $R_{t}$-translate $f_{t}(Z)$ of $f(Z)$ such that $f_{t}(Z)$ is of $R_{t}$-type $(a, 0, c)$ for some $a, c$.

Since $\nu$ is rational nondiscrete, by Lemma 2.2, there exists a sequence of quadratic transforms $R \rightarrow R_{t}$ along $\nu$ such that $x_{t-1}=x_{t}$ and $y_{t-1}=x_{t}\left(y_{t}+\alpha_{t}\right)$ for some

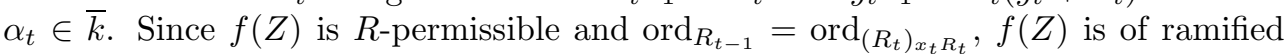
type relative to ord $R_{t-1}$. By Lemma 7.10, after replacing $f(Z)$ with an $R_{t-1}$-translate, and we consider the expansion $F=\sum F_{i j} x_{t-1}^{i} y_{t-1}^{j} \in \widehat{R_{t-1}}=\bar{k}\left[\left[x_{t-1}, y_{t-1}\right]\right]$, we have that if $d=\operatorname{ord}_{R_{t-1}} F, i+j=d$ and $(i, j) \equiv 0(m)$, then $F_{i j}=0$. Let $c$ be the largest $j$ such that there exists $j$ such that $F_{i, j} \neq 0$ and $i+j=d$. Then $f(Z)$ is of $R_{t}$-type $(d, 0, c)$.

Step 2. Assume that $f(Z)$ is of $R$-type $(a, b, c)$ with $b \equiv 0(m)$ and $[b, c] \geq m$. Then there exists a sequence of quadratic transforms $R \rightarrow R_{t}$ along $\nu$, and an $R_{t-1}$-translate $f_{t}(Z)$ of $f(Z)$, such that $f_{t}(Z)$ is of $R_{t}$-type $\left(a_{t}, b_{t}, c_{t}\right)$ with $b_{t} \equiv 0(m)$ and $\left[b_{t}, c_{t}\right]<m$.

The proof of Step 2 follows from two algorithms.

Algorithm 1. Assume that $f(Z)$ is of $R$-type $(a, b, c)$ with $b \equiv 0(m)$ and $[b, c] \geq m$. Then there exists a sequence of quadratic transforms $R \rightarrow R_{e}$ along $\nu$, and an $R_{e-1^{-}}$ translate $f_{e}(Z)$ of $f(Z)$, such that either

1. $f_{e}(Z)$ is of $R_{e}$-type $\left(a_{e}, b_{e}, c_{e}\right)$ with $b_{e} \equiv 0(m)$ and $\left[b_{e}, c_{e}\right]<[b, c]$ or

2. $f_{e}(Z)$ is of $R_{e}$-antitype $\left(b_{e}, a_{e}, d_{e}\right)$ with $a_{e} \equiv 0(m)$ and $d_{e}<m$.

Proof of Algorithm 1.

Since $\nu$ is rational nondiscrete, by Lemma 2.2, there exists a sequence of quadratic transforms along $\nu$,

$$
R=R_{0} \rightarrow \cdots \rightarrow R_{i} \rightarrow R_{i+1}
$$

where $R \rightarrow R_{i}$ is a sequence of $i \geq 0$ quadratic transforms of the type of (12) and $R_{i} \rightarrow R_{i+1}$ is a quadratic transform of the type of (13). Lemma 7.17 implies that 
there exists an $R_{i-1}$-translate $f_{i}(Z)$ of $f(Z)$ such that $f_{i}(Z)$ is of type $\left(a_{i}, b_{i}, c_{i}\right)$ where $b_{i} \equiv 0(m)$ and $c_{i} \leq c$. Now 1 of Lemma 7.19 implies that either there exists an $R_{i}$-translate $f_{i+1}(Z)$ of $f(Z)$ such that $f_{i+1}(Z)$ is of $R_{i+1}$-type $\left(a_{i+1}, b_{i+1}, c_{i+1}\right)$ with $b_{i+1} \equiv 0(m)$ and $\left[b_{i+1}, c_{i+1}\right]<[b, c]$, or $f_{i+1}(Z)$ is of $R_{i+1}$-antitype $\left(b_{i+1}, a_{i+1}, d_{i+1}\right)$ with $a_{i+1} \equiv 0(m)$ and $d_{i+1}<m$. In either case, the conclusions of the algorithm have been reached.

Algorithm 2. Assume that $f(Z)$ is of $R$-antitype $(b, a, d)$ with $a \equiv 0(m)$ and $d<m$. Then there exists a sequence of quadratic transforms $R \rightarrow R_{e}$ along $\nu$, and an $R_{e-1^{-}}$ translate $f_{e}(Z)$ of $f(Z)$ such that either

1. $f_{e}(Z)$ is of $R_{e}$-type $\left(a_{e}, b_{e}, c_{e}\right)$ with $b_{e} \equiv 0(m)$ and $\left[b_{e}, c_{e}\right]<m$ or

2. $f_{e}(Z)$ is of $R_{e}$-antitype $\left(b_{e}, a_{e}, d_{e}\right)$ with $a_{e} \equiv 0(m)$ and $d_{e}<d$.

Proof of Algorithm 2.

Since $\nu$ is rational nondiscrete, by Lemma 2.2, there exists a sequence of quadratic transforms

$$
R=R_{0} \rightarrow \cdots \rightarrow R_{i} \rightarrow R_{i+1}
$$

along $\nu$ where $R \rightarrow R_{i}$ is a sequence of $i \geq 0$ quadratic transforms of the type of (13) and $R_{i} \rightarrow R_{i+1}$ is a quadratic transform of the type of (12). Lemma 7.20 implies that there exists an $R_{i-1}$-translate $f_{i}(Z)$ of $f(Z)$ such that $f_{i}(Z)$ has $R_{i}$-antitype $\left(b_{i}, a_{i}, d_{i}\right)$ with $d_{i} \leq d$ and $a_{i} \equiv 0(m)$. Now 1 of Lemma 7.18 implies that either there exists an $R_{i}$-translate $f_{i+1}(Z)$ of $f(Z)$ such $f_{i+1}(Z)$ has $R_{i+1}$ type $\left(a_{i+1}, b_{i+1}, c_{i+1}\right)$ with $b_{i+1} \equiv 0(m)$ and $\left[b_{i+1}, c_{i+1}\right]<m$, or $f_{i+1}(Z)$ has $R_{i+1}$-antitype $\left(b_{i+1}, a_{i+1}, d_{i+1}\right)$ with $a_{i+1} \equiv 0(m)$ and $d_{i+1}<d_{i} \leq d$. In either case, the conclusions of the algorithm have been reached.

Now we now easily prove Step 2. By successive application of Algorithms 1 and 2 , we either achieve the conclusions of Step 2, or reach an $R_{e}$ such that there exists an $R_{e-1}$-translate $f_{e}(Z)$ of $f(Z)$ such $f_{e}(Z)$ has $R_{e}$-antitype $\left(b_{e}, a_{e}, d_{e}\right)$ with $a_{e} \equiv 0(m)$ and $d_{e}=0$. Then Lemma 7.20 and 1 of Lemma 7.18 imply that after a further sequence of quadratic transforms along $\nu$, the conclusions of Step 2 hold.

Step 3. Assume that $f(Z)$ has $R$-type $(a, b, c)$ with $[b, c]<m$. Then there exists a sequence of quadratic transforms $R \rightarrow R_{t}$ along $\nu$ and an $R_{t-1}$-translate $f_{t}(Z)$ of $f(Z)$ such such that $f_{t}(Z)$ is of $R_{t}$-type $\left(a_{t}, b_{t}, c_{t}\right)$ with $\left[b_{t}, c_{t}\right]=0$.

To prove Step 3, we iterate the following algorithm, which gives a reduction of $[b, c]$.

Since $\nu$ is rational nondiscrete, by Lemma 2.2, there exists a sequence of quadratic transforms along $\nu$

$$
R=R_{0} \rightarrow \cdots \rightarrow R_{i} \rightarrow R_{i+1} \rightarrow \cdots \rightarrow R_{j} \rightarrow R_{j+1}
$$

where $R \rightarrow R_{i}$ is a sequence of $i \geq 0$ quadratic transforms of the type of (12), $R_{i} \rightarrow R_{j}$ is a sequence of $j-i \geq 1$ quadratic transforms of the type of (13) and $R_{j} \rightarrow R_{j+1}$ is a quadratic transform of the type of (12). Lemma 7.17 implies that there exists an $R_{i-1}$-translate $f_{i}(Z)$ of $f(Z)$ such $f_{i}(Z)$ is of $R_{i}$-type $\left(a_{i}, b_{i}, c_{i}\right)$ with 
$\left[b_{i}, c_{i}\right] \leq[b, c]<m$. Now 2 of Lemma 7.19 and Lemma 7.20 implies there exists an $R_{j-1}$ translate $f_{j}(Z)$ of $f(Z)$ such that one of the following holds:

1. $f_{j}(Z)$ is of $R_{j}$-type $\left(a_{j}, b_{j}, c_{j}\right)$ with $\left[b_{j}, c_{j}\right] \leq \max \left\{0,\left[b_{i}, c_{i}\right]-1\right\}$ or

2. $f_{j}(Z)$ is of $R_{j}$-antitype $\left(b_{j}, a_{j}, d_{j}\right)$ with $d_{j}<\frac{m}{p}$ or

3. $f_{j}(Z)$ is of $R_{j}$-antitype $\left(b_{j}, a_{j}, d_{j}\right)$ with $d_{j}=\frac{m}{p}$ and $a_{j}+b_{j}+\frac{m}{p} \not \equiv 0(m)$.

Finally, Lemma 7.17 and 2 of Lemma 7.18 imply that there exists an $R_{j}$-translate $f_{j+1}(Z)$ of $f(Z)$ such $f_{j+1}(Z)$ is of $R_{j+1}$-type $\left(a_{j+1}, b_{j+1}, c_{j+1}\right)$ with $\left[b_{j+1}, c_{j+1}\right]<$ $[b, c]$.

Step 4. Suppose that there exists an $R_{t-1}$ translate $f_{t}(Z)$ of $f(Z)$ such that $f_{t}(Z)$ is of $R_{t}$-type $\left(a_{t}, b_{t}, c_{t}\right)$ with $\left[b_{t}, c_{t}\right]=0$. Then there exists an $R_{t}$-translate $f_{t}(Z)$ of $f(Z)$ which is of $R_{t}$-stable-type $\left(a_{t}, b_{t}, c^{\prime}\right)$ where $c^{\prime} \leq c_{t}$.

Step 4 follows from Lemma 7.15.

Step 5. Let $t$ be as in Step 4. After any further sequence of quadratic transforms $R_{t} \rightarrow R_{i}$ along $\nu$, and making an appropriate $R_{i}$-translate $f_{i}(Z)$ of $f(Z), f_{i}(Z)$ is of $R_{i}$-stable-type.

Step 5 follows from Lemma 7.16.

8. Reduction of order of a polynomial. Theorem 8.1 finds a particular substitution which gives a reduction of order of a polynomial. This is used later in Theorem 9.7 to show that after a sequence of blow ups of points along a valuation, we reduce to a good point; that is, a point from which a reduction of multiplicity can be obtained by only blowing up curves. The property of being a good point may not be preserved by further blow ups of points.

THEOREM 8.1. Let $R_{0}$ be a two dimensional regular local ring, containing an algebraically closed field $\bar{k}$ of characteristic $p$ which is isomorphic to the residue field of $R_{0}$. Let $K$ be the quotient field of $R_{0}$. Let $f(Z) \in R_{0}[Z]$ be monic of degree $n>0$. Let $\nu$ be a 0-dimensional valuation of $K$ which dominates $R_{0}$. Let

$$
R_{0} \rightarrow R_{1} \rightarrow \cdots \rightarrow R_{k} \rightarrow \cdots
$$

be the infinite sequence of quadratic transforms along $\nu$. Then there exist regular parameters $x_{0}, y_{0}$ in $R_{0} k \in \mathbb{N}$, and $r \in \hat{R}_{k}$ such that $f(Z+r)=Z^{n}$ or there exists nonnegative integers $u$ and $v$ such that upon setting $g(Z)=\left(x_{k}^{u} y_{k}^{v}\right)^{-n} f\left(x_{k}^{u} y_{k}^{v} Z+r\right)$ we have that $g(Z) \in \hat{R}_{k}[Z]$ and $0<\operatorname{ord}_{\hat{R}_{k}} g(Z)<n$, where $x_{k}, y_{k}$ are the canonical parameters in $R_{k}$ determined by $x_{0}, y_{0}$.

We will prove this theorem in the essential case when $\nu$ is rational nondiscrete. This is by far the hardest case, and is the situation where differences between characteristic zero and $p$ are most evident. The remaining cases of valuations are essentially "toric" (The irrational case is for instance handled in Section 1 of [1]).

In this section, we will use the following notation. Suppose that $R$ is a two dimensional regular local ring containing an algebraically closed field $\bar{k}$ of characteristic $p>0$ which is isomorphic to its residue field. Let $K$ be the quotient field of $R$. Suppose that $\nu$ is a rational nondiscrete valuation of $K$ dominating $R$. Let $x_{0}, y_{0}$ be regular parameters in $R$, and let

$$
R=R_{0} \rightarrow R_{1} \rightarrow \cdots \rightarrow R_{k} \rightarrow \cdots
$$


be the infinite sequence of quadratic transforms along $\nu$. Let $x_{k}, y_{k}$ be the canonical coordinates in $R_{k}$ with respect to $x, y$. Let $S=R_{x R}$ and $S_{k}=\left(R_{k}\right)_{x_{k} R_{k}}$ for $k \geq 0$. Let $\bar{I}$ be the set of integers $k$ such that

$$
x_{k-1}=x_{k}, y_{k-1}=x_{k}\left(y_{k}+\alpha_{k}\right) \text { with } 0 \neq \alpha_{k} \in \bar{k} \text {. }
$$

For $t \in \bar{I}$, let $\bar{I}(t)$ be the set of all $k \in \bar{I}$ such that $k \geq t$. Since $\nu$ is rational nondiscrete, $\bar{I}$ is an infinite set (by Lemma 2.2 ).

Let $f(Z) \in K[z]$ be monic of degree $n>0$. Let $L$ be a splitting field of $f(Z)$ over $K$, so that we have $L=K\left(z_{1}, \ldots, z_{n}\right)$ with $f(Z)=\left(Z-z_{1}\right) \cdots\left(Z-z_{n}\right)$. We define

$$
D_{K}(f(Z))=\prod_{i \neq j}\left(y_{i}-y_{j}\right)^{d}
$$

where $y_{1}, \ldots, y_{m}$ are the distinct elements amongst the elements $z_{1}, \ldots, z_{n}$, and $d=$ $[L: K]$. Note that if $R$ is any normal domain with quotient field $K$ such that $f(Z) \in R[Z]$, then $D_{K}(f(Z)) \in R$.

Let $g(Z)$ be another monic polynomial of positive degree in $Z$ with coefficients in $K$. We define

$$
D(g(Z), f(Z))=\prod_{i=1}^{n} g\left(z_{i}\right) .
$$

Observe that if $R$ is any normal domain with quotient field $K$ such that $f(Z), g(Z) \in$ $R[Z]$, then $D(g(Z), f(Z)) \in R$.

THEOREM 8.2. Assume that $\nu$ is rational nondiscrete. Let $L$ be a finite normal extension of $K$ such that $\nu$ does not split in $L$. Let $[L: K]=p^{\prime} q$ where $p \nmid \chi q$ and $p^{\prime}$ is a power of $p$. Then

1. There exists a unique subfield $K^{\prime}$ of $L$ containing $K$ such that $\left[K^{\prime}: K\right]=q$. $K^{\prime}$ is a cyclic Galois extension of $K$ and $\nu$ does not split in $K^{\prime}$.

2. There exists $t \in \bar{I}$ such that for all $k \in \bar{I}(t)$,

a) $S_{k}$ is totally ramified in $L$,

b) the integral closure $R_{k}^{\prime}$ of $R_{k}$ in $K^{\prime}$ is a regular local ring, with regular parameters $x_{k}^{\prime}, y_{k}^{\prime}$, which are canonical with respect to $x_{t}^{\prime}, y_{t}^{\prime},\left(x_{k}^{\prime}\right)^{q}=$ $\delta_{k} x_{k}$ where $\delta_{k} \in R_{k}$ is a unit and $\left(x_{k}^{\prime}, y_{k}\right) R_{k}^{\prime}=M\left(R_{k}^{\prime}\right)$,

c) $S_{k}^{\prime}=\left(R_{k}^{\prime}\right)_{x_{k}^{\prime} R_{k}^{\prime}}$ is totally ramified in $L$.

3. Let $k \in \bar{I}(t)$ and let $g(Z) \in R_{k}[Z]$ be a monic irreducible polynomial of degree $d>1$ such that $g(Z)=0$ for some $z \in K^{\prime}$ and $D_{K}(g(Z))$ is an $R_{k}$-monomial in $x_{k}$. Let $f(Z)=g(Z)^{e}$ where $e$ is a positive integer and let $n=$ de. Then there exists $r \in R_{k}$ and a positive integer a with $a \not \equiv 0(n)$ such that for any $r^{*} \in R_{k}$ with $\operatorname{ord}_{S_{k}} r^{*} \geq \frac{a}{n}$ we have that $\frac{f\left(r+r^{*}\right)}{x^{a}}$ is a unit in $R_{k}$.

4. Let $k \in \bar{I}(t)$ and $g(Z) \in R_{k}[Z]$ be a monic irreducible polynomial of degree $d>0$. Let $f^{\prime}(Z) \in R_{k}^{\prime}[Z]$ be a monic irreducible polynomial of degree $m$ such that $f^{\prime}(Z)$ divides $g^{\prime}(Z)$ in $K^{\prime}[Z]$, and $f^{\prime}(z)=0$ for some $z \in L$. Then $m$ is the highest power of $p$ which divides $d$. Let $f(Z)=g(Z)^{e}$ where $e$ is a positive integer and let $n=$ de. Assume that $D_{K}(g(Z))$ is an $R_{k}$-monomial in $x_{k}$ and there exist nonnegative integers $a^{\prime}$ and $c^{\prime}$ and $r^{\prime} \in R_{k}^{\prime}$ such that $\operatorname{ord}_{S_{k}^{\prime}} f^{\prime}\left(r^{\prime}\right)=a^{\prime}, \operatorname{ord}_{R_{k}^{\prime} / x_{k}^{\prime} R_{k}^{\prime}}\left(\frac{f\left(r^{\prime}\right)}{\left(x_{k}^{\prime}\right)^{a^{\prime}}}\right)=c^{\prime},\left(a^{\prime}, c^{\prime}\right) \not \equiv 0(m)$ and $c^{\prime} \leq \frac{m}{p}$. Then there exist nonnegative integers $a$ and $c$ and $r \in R_{k}$ such that $(a, c) \not \equiv 0(n)$ and $c \leq \frac{n}{p}$ and such that for any $r^{*} \in R_{k}$ with $\operatorname{ord}_{S_{k}} r^{*} \geq \frac{a}{n}$ we have that $\operatorname{ord}_{S_{k}} f\left(r+r^{*}\right)=a$ and $\operatorname{ord}_{R_{k} / x_{k} R_{k}} \frac{f\left(r+r^{*}\right)}{x_{k}^{a}}=c$. 
This is proven in Lemma 2.8, Theorem 2.9 and Lemma 2.4 of [6]. Conclusions 1 and 2 are a case of Abhyankar's generalization of Jung's theorem on ramification to positive characteristic. This result is also interpreted and discussed in [23]. An essential ingredient in Abhyankar's proof is a Theorem of Krull on the large ramification group of a valuation ([36] and Section 12 of Chapter 6 of [47]). The proofs of 3 and 4 involve an ingenious use of Galois theory.

LEMma 8.3. (Lemma 2.5 [6]) Let $f(Z) \in R[Z]$ be a monic polynomial of degree $n$, and let $L$ be a finite normal extension of $K$ such that $S$ does not split in $L$, and $f(Z)$ factors into linear factors in $L[Z]$. Write $f(Z)=\prod_{i=1}^{v} g_{i}(Z)^{e(i)}$ where $g_{i}(Z) \in R[Z]$ are the distinct monic irreducible factors of $f(Z)$ in $K[Z]$. Let $f_{i}(Z)=g_{i}(Z)^{e(i)}$ for $1 \leq i \leq v$. For $1 \leq i \leq v$, let $d(i)$ be the degree of $g_{i}(Z)$ and suppose that $d(i)>1$ for all $i$. Let $n(i)=d(i) e(i)$ and $n=n(1)+\cdots+n(v)$. Assume that for $1 \leq i \leq v$ there exist $a(i), c(i) \in \mathbb{N}$ and $r_{i} \in R$ such that $(a(i), c(i)) \not \equiv 0(n(i))$ and $c(i)<n(i)$ and for any $r_{i}^{*} \in R$ with $\operatorname{ord}_{S}\left(r_{i}^{*}\right) \geq \frac{a(i)}{n(i)}$, we have that $\operatorname{ord}_{S}\left(f_{i}\left(r_{i}+r_{i}^{*}\right)\right)=a(i)$ and $\operatorname{ord}_{R / x R}\left(\frac{f_{i}\left(r_{i}+r_{i}^{*}\right)}{x^{a(i)}}\right)=c(i)$. Also assume that $D\left(g_{i}(Z), g_{j}(Z)\right)$ is an $R$-monomial in $x$ whenever $1 \leq i \leq v, 1 \leq j \leq v$ and $i \neq j$. Then there exists $r \in R$ such that upon letting

$$
F(Z)=f(Z+r)=Z^{n}+F_{1} Z^{n-1}+\cdots+F_{n}
$$

with $F_{1}, \ldots, F_{n} \in R$, we either have that

1. There exists an integer $u$ such that $0<u<n, F_{u}$ is an $R$-monomial in $x$, $\operatorname{ord}_{S} F_{j} \geq \frac{j}{u} \operatorname{ord}_{S} F_{u}$ for $1 \leq j \leq u$ and $\operatorname{ord}_{S} F_{j}>\frac{j}{u} \operatorname{ord}_{S} F_{u}$ for $u<j \leq n$ or

2. There exist $a, c \in \mathbb{N}$ such that $(a, c) \not \equiv 0(n), c \leq c(1)+\cdots+c(v)$, $\operatorname{ord}_{S}\left(F_{n}\right)=a$, $\operatorname{ord}_{R / x R}\left(\frac{F_{n}}{x^{a}}\right)=c$, and $\operatorname{ord}_{S}\left(F_{j}\right) \geq \frac{j a}{n}$ for $1 \leq j \leq n$.

Proof. After permuting $1, \ldots, v$, we may assume that $\frac{a(1)}{n(1)} \geq \frac{a(i)}{n(i)}$ for $1<i \leq v$. Let $r=r_{1}$, and

$$
F(Z)=f(Z+r)=Z^{n}+F_{1} Z^{n-1}+\cdots+F_{n}
$$

with $F_{1}, \ldots, F_{n} \in R$. For $1 \leq i \leq v$, let $g_{i}^{\prime}(Z)=g_{i}(Z+r), f_{i}^{\prime}(Z)=g_{i}^{\prime}(Z)^{e(i)}$, $y_{i}=z_{i}-r, s_{i}=r_{i}-r$ and $\bar{b}(i)=\operatorname{ord}_{S} f_{i}^{\prime}(0)$. Then $F(Z)=f_{1}^{\prime}(Z) \cdots f_{v}^{\prime}(Z)$ and for each $i, g_{i}^{\prime}(Z)$ is a monic irreducible polynomial of degree $d(i)$ in $R[Z], y_{i} \in L$, $g_{i}^{\prime}\left(y_{i}\right)=0, s_{i} \in R$ and

$$
\begin{aligned}
& \operatorname{ord}_{S}\left(f_{i}^{\prime}\left(s_{i}+s_{i}^{*}\right)\right)=a(i) \\
& \text { and } \operatorname{ord}_{R / x R}\left(\frac{f_{i}^{\prime}\left(s_{i}+s_{i}^{*}\right)}{x^{a(i)}}\right)=c(i) \text { for all } s_{i}^{*} \in R \text { with } \operatorname{ord}_{S}\left(s_{i}^{*}\right) \geq \frac{a(i)}{n(i)} .
\end{aligned}
$$

We have that $s_{1}=0$, and thus

$$
\operatorname{ord}_{S}\left(f_{1}^{\prime}(0)\right)=a(1)=b(1) .
$$

For $1<i \leq v$ we have that $D\left(f_{i}^{\prime}(Z), f_{1}^{\prime}(Z)\right)=D\left(f_{i}(Z), f_{1}(Z)\right)=$ $\left(D\left(g_{i}(Z), g_{1}(Z)\right)\right)^{e(i) e(1)}$ and hence $D\left(f_{i}^{\prime}(Z), f_{1}^{\prime}(Z)\right)$ is an $R$-monomial in $x$. Let $T$ be the integral closure of $S$ in $L$. By our assumption that $S$ does not split in $L$, we have that $T$ is a one dimensional regular local ring, and for any $K$-automorphism $G$ of $L$, we have that $G(T)=T$ and hence $\operatorname{ord}_{T}(G(y))=\operatorname{ord}_{T}(y)$ for all $y \in L$. We also have that $\operatorname{ord}_{T}(y)=\operatorname{ord}_{S}(y) \operatorname{ord}_{T}(x)$ for $y \in K$. Since $L$ is a finite normal extension of $K$, there exist $K$-automorphisms $G_{i j}$ of $L$ such that upon letting $y_{i j}=G_{i j}\left(y_{i}\right)$ we have 
that $g_{i}^{\prime}(Z)=\left(Z-y_{i 1}\right) \cdots\left(Z-y_{i d(i)}\right)$ for $1 \leq i \leq v$. We have that $\operatorname{ord}_{T}\left(y_{i j}\right)=\operatorname{ord}_{T}\left(y_{i}\right)$ for $1 \leq i \leq v$ and $1 \leq j \leq d(i)$ and

$$
f_{i}^{\prime}(Z)=\prod_{j=1}^{d(i)}\left(Z-y_{i j}\right)^{e(i)}=Z^{n(i)}+\sum_{k=1}^{n(i)} f_{i k}^{\prime} Z^{n(i)-k}
$$

where $f_{i k}^{\prime} \in R$ for $1 \leq i \leq v$ and $1 \leq k \leq n(i)$. Since $f_{i}^{\prime}(0)=f_{\text {in(i) }}^{\prime}=$ $(-1)^{n(i)}\left(\prod_{j=1}^{d(i)} y_{i j}\right)^{e(i)}$, we have

$$
\operatorname{ord}_{T}\left(y_{i j}\right)=\operatorname{ord}_{T}\left(y_{i}\right)=\frac{b(i)}{n(i)} \operatorname{ord}_{T}(x) \text { for } 1 \leq i \leq v \text { and } 1 \leq j \leq d(i) .
$$

Since $f_{i k}^{\prime}$ is the elementary symmetric function of degree $k$ in the $y_{i j}$, which is homogeneous in the $y_{i j}$ of degree $k$, and by (29), we have that

$$
\operatorname{ord}_{S}\left(f_{i k}^{\prime}\right) \geq k \frac{b(i)}{n(i)} \text { for } 1 \leq i \leq v \text { and } 1 \leq k \leq n(i)
$$

Let $i$ be any integer such that $1 \leq i \leq v$ and $\frac{b(i)}{n(i)}<\frac{a(1)}{n(1)}$. By (28) and (29), we have that $b(1)=a(1), i \neq 1, \frac{b(i)}{n(i)}<\frac{b(1)}{n(1)}$ and $\operatorname{ord}_{T}\left(y_{1 j}\right)=\frac{b(1)}{n(1)} \operatorname{ord}_{T}(x)$ for $1 \leq j \leq d(1)$. Since $\operatorname{ord}_{S} f_{i}^{\prime}(0)=b(i)$ and

$$
f_{i}^{\prime}\left(y_{1 j}\right)=y_{1 j}^{n(i)}+\sum_{k=1}^{n(i)} f_{i k}^{\prime} y_{1 j}^{n(i)-k} \text { for } 1 \leq j \leq d(1),
$$

we get by (30) that for $1 \leq k \leq n(i)$,

$$
\begin{aligned}
\operatorname{ord}_{T}\left(f_{i k}^{\prime} y_{1 j}^{n(i)-k}\right) & =\operatorname{ord}_{T}\left(f_{i k}^{\prime}\right)+(n(i)-k) \operatorname{ord}_{T}\left(y_{1 j}\right) \\
& \geq k \frac{b(i)}{n(i)} \operatorname{ord}_{T}(x)+(n(i)-k) \frac{b(1)}{n(1)} \operatorname{ord}_{T}(x) \\
& \text { and } \operatorname{ord}_{T}\left(y_{1 j}^{n(i)}\right)=n(i) \frac{b(1)}{n(1)} \operatorname{ord}_{T}(x) .
\end{aligned}
$$

Thus ord $f_{i}^{\prime}\left(y_{1 j}\right)=\operatorname{ord}_{T}\left(f_{\text {in(i) }}^{\prime}\right)=\operatorname{ord}_{T} f_{i}^{\prime}(0)=\operatorname{ord}_{T}\left(x^{b(i)}\right)$ and $\operatorname{ord}_{T}\left(f_{i}^{\prime}\left(y_{1 j}\right)-\right.$ $\left.f_{i}^{\prime}(0)\right)>\operatorname{ord}_{T}\left(x^{b(i)}\right)$ for $1 \leq j \leq d(1)$. Thus $\frac{f_{i}^{\prime}\left(y_{1 j}\right)}{x^{b(i)}} \in T, \frac{f_{i}^{\prime}(0)}{x^{b(i)}} \in T$ and $\frac{f_{i}^{\prime}\left(y_{1 j}\right)}{x^{b(i)}} \equiv$ $\frac{f_{i}^{\prime}(0)}{x^{b(i)}} \bmod Q$ for $1 \leq j \leq d(1)$, where $Q$ is the maximal ideal of $T$, and hence

$$
\prod_{j=1}^{d(1)}\left(\frac{f_{i}^{\prime}\left(y_{1 j}\right)}{x^{b(i)}}\right)^{e(1)} \equiv\left(\frac{f_{i}^{\prime}(0)}{x^{b(i)}}\right)^{n(1)} \bmod Q .
$$

We have that

$$
\prod_{j=1}^{d(1)} f_{i}^{\prime}\left(y_{1 j}\right)^{e(1)}=D\left(f_{i}^{\prime}(Z), f_{1}^{\prime}(Z)\right)=t x^{b^{\prime}}
$$

where $t$ is a unit in $R$ and $b^{\prime}$ is a nonnegative integer. Hence

$$
t x^{b^{\prime}-b(i) n(1)} \equiv\left(\frac{f_{i}^{\prime}(0)}{x^{b(i)}}\right)^{n(1)} \bmod Q .
$$


Since $t x^{b^{\prime}-b(i) n(1)}$ and $\left(\frac{f_{i}^{\prime}(0)}{x^{b(i)}}\right)^{n(1)}$ are in $K$ and $K \cap Q=x S$, we obtain

$$
t x^{b^{\prime}-b(i) n(1)}-\left(\frac{f_{i}^{\prime}(0)}{x^{b(i)}}\right)^{n(1)} \in x S .
$$

Since $f_{i}^{\prime}(0) \in R$ and $\operatorname{ord}_{S}\left(f_{i}^{\prime}(0)\right)=b(i)$, we obtain $\frac{f_{i}^{\prime}(0)}{x^{((i)}} \in R$ and $\frac{f_{i}^{\prime}(0)}{\left.x^{(i)}\right)}$ is a unit in $S$. Thus by (32), we get that $t x^{b^{\prime}-b(i) n(1)}$ is a unit in $S$. Since $t$ is a unit in $R$, we have that $b^{\prime}-b(i) n(1)=0$ and hence by 32 we obtain $t-\left(\frac{f_{i}^{\prime}(0)}{x^{b(i)}}\right)^{n(1)} \in(x S) \cap R=x R$. Since $t$ is a unit in $R$ we conclude that $\frac{f_{i}^{\prime}(0)}{x^{b(2)}}$ is a unit in $R$. Thus we have shown that:

$$
\begin{aligned}
& \text { if } i \text { is any integer such that } 1 \leq i \leq v \text { and } \frac{b(i)}{n(i)}<\frac{a(1)}{n(1)}, \\
& \text { then } \frac{f_{i}^{\prime}(0)}{x^{b(i)}} \text { is a unit in } R \text {. }
\end{aligned}
$$

Now let $i$ be an integer such that $1 \leq i \leq v$ and $\frac{b(i)}{n(i)} \geq \frac{a(1)}{n(1)}$. Since $\frac{a(1)}{n(1)} \geq \frac{a(i)}{n(i)}$ we have $b(i) \geq a(i)$, and by 30 we have that $\operatorname{ord}_{S}\left(f_{i k}^{\prime}\right) \geq k \frac{a(i)}{n(i)}$ for $1 \leq k \leq n(i)$. Setting $s_{i}^{*}=0$ in $(27)$, we $\operatorname{get} \operatorname{ord}_{S}\left(f_{i}^{\prime}\left(s_{i}\right)\right)=a(i)$. We have

$$
f_{i}^{\prime}\left(s_{i}\right)=s_{i}^{n(i)}+\sum_{k=1}^{n(i)} f_{i k}^{\prime} s_{i}^{n(i)-k}
$$

and hence (by an analysis similar to (31)) we must have $\operatorname{ord}_{S}\left(s_{i}\right) \geq \frac{a(i)}{n(i)}$. Thus after taking $s_{i}^{*}=-s_{i}$ in $(27)$, we get that $\operatorname{ord}_{S}\left(f_{i}^{\prime}(0)\right)=a(i)$ and $\operatorname{ord}_{R / x R}\left(\frac{f_{i}^{\prime}(0)}{x^{a(i)}}\right)=c(i)$. Since $\frac{b(i)}{n(i)} \geq \frac{a(1)}{n(1)} \geq \frac{a(i)}{n(i)}$ and $b(i)=\operatorname{ord}_{S}\left(f_{i}^{\prime}(0)\right)=a(i)$, we get that $\frac{a(i)}{n(i)}=\frac{a(1)}{n(1)}$. Thus we have shown that

$$
\begin{aligned}
& \text { if } i \text { is any integer such that } 1 \leq i \leq v \text { and } \frac{b(i)}{n(i)} \geq \frac{a(1)}{n(1)} \text {, then } \\
& \operatorname{ord}_{S}\left(f_{i}^{\prime}(0)\right)=a(i), \operatorname{ord}_{R / x R}\left(\frac{f_{i}^{\prime}(0)}{x^{a(i)}}\right)=c(i), b(i)=a(i) \text { and } \frac{a(i)}{n(i)}=\frac{a(1)}{n(1)} .
\end{aligned}
$$

We will now show that if $\frac{b(i)}{n(i)}<\frac{a(1)}{n(1)}$ for some $i$ then condition 1 of the conclusions of the theorem hold, and if $\frac{b(i)}{n(i)} \geq \frac{a(1)}{n(1)}$ for all $i$ with $1 \leq i \leq v$, then condition 2 of the conclusions of the theorem hold. This will complete the proof.

First suppose that $\frac{b(i)}{n(i)}<\frac{a(1)}{n(1)}$ for some $i$. Let $a^{\prime}=\min \left\{\frac{b(1)}{n(1)}, \ldots, \frac{b(v)}{n(v)}\right\}$, let $V$ be the set of all integers $i$ such that $1 \leq i \leq v$ and $\frac{b(i)}{n(i)}=a^{\prime}$, and let $V^{\prime}$ be the set of all integers $i$ such that $1 \leq i \leq v$ and $i \notin V$. Then $V \neq \emptyset$, and by (28), we have that $1 \in V^{\prime}$, so that $V^{\prime} \neq \emptyset$. Let

$$
u=\sum_{i \in V} n(i) \text { and } a=\sum_{i \in V} b(i) .
$$

Then $0<u<n$ and $a=u a^{\prime}$. Let

$$
\begin{gathered}
A(Z)=\prod_{i \in V} f_{i}^{\prime}(Z)=Z^{u}+\sum_{j=1}^{u} A_{j} Z^{u-j} \\
B(Z)=\prod_{i \in V^{\prime}} f_{i}^{\prime}(Z)=Z^{n-u}+\sum_{j=1}^{n-u} B_{j} Z^{n-u-j}
\end{gathered}
$$


with $A_{1}, \ldots, A_{u}, B_{1}, \ldots, B_{n-u} \in R$. We have that

$$
A_{u}=A(0)=\prod_{i \in V} f_{i}^{\prime}(0)
$$

By (33), we know that $\frac{f_{i}^{\prime}(0)}{x^{b(i)}}$ is a unit in $R$ for all $i \in V$. Thus

$$
\operatorname{ord}_{S}\left(A_{u}\right)=u a^{\prime} \text { and } \frac{A_{u}}{x^{u a^{\prime}}} \text { is a unit in } R \text {. }
$$

Since $\frac{b(i)}{n(i)}=a^{\prime}$ for $i \in V$ and $\frac{b(i)}{n(i)}>a^{\prime}$ for $i \in V^{\prime}$, by (29) we obtain $\operatorname{ord}_{T}\left(y_{i j}\right)=$ $a^{\prime} \operatorname{ord}_{T}(x)$ whenever $i \in V$ and $1 \leq j \leq d(i)$, and $\operatorname{ord}_{T}\left(y_{i j}\right)>a^{\prime} \operatorname{ord}_{T}(x)$ whenever $i \in V^{\prime}$ and $1 \leq j \leq d(i)$. Since

$$
A(Z)=\prod_{i \in V} \prod_{j=1}^{d(i)}\left(Z-y_{i j}\right)^{e(i)}
$$

and

$$
B(Z)=\prod_{i \in V^{\prime}} \prod_{j=1}^{d(i)}\left(Z-y_{i j}\right)^{e(i)}
$$

we have that

$$
\operatorname{ord}_{S}\left(A_{j}\right) \geq j a^{\prime} \text { for } 1 \leq j \leq u \text { and } \operatorname{ord}_{S}\left(B_{j}\right)>j a^{\prime} \text { for } 1 \leq j \leq n-u \text {. }
$$

We have that $F(Z)=A(Z) B(Z)$. Setting $A_{0}=1$, we have

$$
F_{j}= \begin{cases}A_{j}+\sum_{k=1}^{\min \{j, n-u\}} B_{k} A_{j-k} & \text { for } 1 \leq j \leq u \\ \sum_{k=1}^{\min \{u, n-j\}} B_{j-u+k} A_{u-k} & \text { for } u<j \leq n .\end{cases}
$$

It follows from (35), (36) and (37) that $\operatorname{ord}_{S}\left(F_{u}\right)=u a^{\prime}, \frac{F_{u}}{x^{u a^{\prime}}}$ is a unit in $R, \operatorname{ord}_{S}\left(F_{j}\right) \geq$ $\frac{j}{u} \operatorname{ord}_{S}\left(F_{u}\right)$ for $1 \leq j \leq u$, and $\operatorname{ord}_{S}\left(F_{j}\right)>\frac{j}{u} \operatorname{ord}_{S}\left(F_{u}\right)$ for $u<j \leq n$.

Finally, suppose that $\frac{b(i)}{n(i)} \geq \frac{a(1)}{n(1)}$ for $1 \leq i \leq v$. by (34) we obtain $\operatorname{ord}_{S}\left(f_{i}^{\prime}(0)\right)=$ $a(i), \operatorname{ord}_{R / x}\left(\frac{f_{i}^{\prime}(0)}{x^{a(i)}}\right)=c(i)$ and $\frac{b(i)}{n(i)}=\frac{a(i)}{n(i)}=\frac{a(1)}{n(1)}$ for $1 \leq i \leq v$. Let $a=a(1)+\cdots+a(v)$ and $c=c(1)+\cdots+c(v)$. Since $F_{n}=F(0)=f_{1}^{\prime}(0) \cdots f_{v}^{\prime}(0)$, we get that $\operatorname{ord}_{S}\left(F_{n}\right)=a$ and $\operatorname{ord}_{R / x}\left(\frac{F_{n}}{x^{a}}\right)=c$. Let $\lambda$ be the common ratio $\lambda=\frac{a(i)}{n(i)}=\frac{b(i)}{n(i)}$ for $1 \leq i \leq v$. Substituting $a(i)=\lambda n(i)$ into $\frac{a}{n}$, we obtain $\frac{a(i)}{n(i)}=\frac{a}{n}$ for $1 \leq i \leq v$. Hence by (29), we get that $\operatorname{ord}_{T}\left(y_{i j}\right)=\frac{a}{n} \operatorname{ord}_{T}(x)$ for $1 \leq i \leq v$ and $1 \leq j \leq d(i)$. Since

$$
\left.F(Z)=\prod_{i=1}^{v} \prod_{j=1}^{d(i)}\left(Z-y_{i j}\right)\right)^{e(i)}
$$

we deduce that $\operatorname{ord}_{S}\left(F_{k}\right) \geq k \frac{a}{n}$ for $1 \leq k \leq n$. Since $c(i)<n(i)$ for $1 \leq i \leq v$ we get that $c<n$. If $c \neq 0$, then we have $(a, c) \not \equiv 0(n)$. If $c=0$ then $c(1)=0$ and hence $a(1) \not \equiv 0(n(1))$, so that $a \not \equiv 0(n)$ since $\frac{a}{n}=\frac{a(1)}{n(1)}$. Thus in both cases $(a, c) \not \equiv 0(n)$.

Lemma 8.4. Let $F(Z) \in R[Z]$ be a monic polynomial of degree $n>0$. Assume

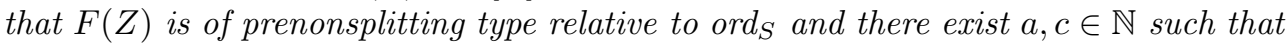


$\operatorname{ord}_{x R} F(0)=a, \operatorname{ord}_{R / x R} \frac{F(0)}{x^{a}}=c,(a, c) \not \equiv 0(n)$ and $c \leq \frac{n}{2}$. Then there exist $k, d, e \in \mathbb{N}$ and an $R_{k}$-translate $F^{\prime}(Z)$ of $F(Z)$ such that upon letting $f(Z)=\frac{F^{\prime}\left(x_{k}^{d} y_{k}^{e} Z\right)}{\left(x_{k}^{d} y_{k}^{e}\right)^{n}}$, we have that $k \leq \frac{n}{2}, f(Z) \in R_{k}[Z]$ and $0<\operatorname{ord}_{R_{k}} f(Z)<n$.

This is Lemma 2.6 [6]. Since $\nu$ is rational nondiscrete, there exists a sequence of quadratic transforms

$$
R=R_{0} \rightarrow R_{1} \rightarrow \cdots \rightarrow R_{i} \rightarrow R_{i+1}
$$

where $i \geq 0$ and $R_{j}$ has canonical coordinates $x_{i}, y_{i}$ for $0 \leq j \leq i+1$ such that $R_{j} \rightarrow R_{j+1}$ is of the type of (13) for $j<i$ and $R_{i} \rightarrow R_{i+1}$ is of the type of (12). A direct calculation shows that the conclusions of the lemma hold with $k=i+1$.

LEMMA 8.5. Let $F(Z)=Z^{n}+F_{1} Z^{n-1}+\cdots+F_{n} \in F[Z]$ with $n>0$ and $F_{1}, \ldots, F_{n} \in R$. Assume that there exists an integer $u$ with $0<u<n$ such that $F_{u}$ is an $R$-monomial in $x$, $\operatorname{ord}_{S} F_{j} \geq \frac{j}{u} \operatorname{ord}_{S} F_{u}$ for $1 \leq j \leq u$ and $\operatorname{ord}_{S} F_{j}>\frac{j}{u} \operatorname{ord}_{S} F_{u}$ for $u<j \leq n$. Let d be the greatest integer such that $d u \leq \operatorname{ord}_{S} F_{u}$, and let $f(Z)=\frac{f\left(x^{d} Z\right)}{x^{n d}}$. Then $f(Z) \in R[Z]$ and $0<\operatorname{ord}_{R} f(Z)<n$.

This is Lemma $2.7[6]$.

THEOREM 8.6. Assume that $\nu$ is rational nondiscrete. Suppose that $R$ is an excellent local ring (in addition to our other assumptions), and $L$ is a finite normal extension of $K$ such that $V$ does not split in $L$. Let $[L: K]=p^{\prime} q$ where $p \nmid q$ and $p^{\prime}$ is a power of $p$, and let $K^{\prime}$ be the intermediate field of Theorem 8.2. Let $g(Z) \in R[Z]$ be a monic irreducible polynomial of degree $d$, and suppose that $g(z)=0$ for some $z \in L$. Let $f(Z)=g(Z)^{e}$ where $e$ is a positive integer, and let $n=$ de. Then there exists $t^{\prime} \in \bar{I}$ such that for each $k \in \bar{I}\left(t^{\prime}\right)$ there exists $r_{k} \in R_{k}$ and nonnegative integers $a(k)$ and $c(k)$ such that $(a(k), c(k)) \not \equiv 0(n), c(k)=0$ if $d \not \equiv 0(p), c(k) \leq \frac{n}{p}$ if $d \equiv 0(p)$, and $\operatorname{ord}_{S_{k}} f\left(r_{k}+r_{k}^{*}\right)=a(k)$ and $\operatorname{ord}_{R_{k} / x_{k} R_{k}} \frac{f\left(r_{k}+r_{k}^{*}\right)}{x^{a(k)}}=c(k)$ for all $r_{k}^{*} \in R_{k}$ with $\operatorname{ord}_{S_{k}} r_{k}^{*} \geq \frac{a(k)}{n}$.

Proof. Let notation be as in Theorem 8.2. In particular, let $t$ be as in 2 of Theorem 8.2. By embedded principalization of ideals in dimension two, there exists $t^{\prime \prime} \in \bar{I}(t)$ such that $D_{K}(g(Z))$ is an $R_{k}$-monomial in $x_{k}$ for all $k \in \bar{I}\left(t^{\prime \prime}\right)$. Let $m=\left[K^{\prime}(z): K^{\prime}\right]$, and let $f^{\prime}(Z)$ be the minimal monic polynomial of $z$ over $K^{\prime}$.

First suppose that $m=1$. Then $z \in K^{\prime}$ and hence $d \not \equiv 0(p)$. By 3 of Theorem 8.2 , we have that for each $k \in \bar{I}\left(t^{\prime \prime}\right)$ there exists $r_{k} \in R_{k}$ and a positive integer $a(k)$ with $a(k) \not \equiv 0(n)$ such that for all $r_{k}^{*} \in R_{k}$ with $\operatorname{ord}_{S_{k}} r_{k}^{*} \geq \frac{a(k)}{n}$ we have that $\frac{f\left(r_{k}+r_{k}^{*}\right)}{x_{k}^{a(k)}}$ is a unit in $R_{k}$, and hence $\operatorname{ord}_{S_{k}} f\left(r_{k}+r_{k}^{*}\right)=a(k)$ and $\operatorname{ord}_{R_{k} / x_{k} R_{k}} \frac{f\left(r_{k}+r_{k}^{*}\right)}{x_{k}^{a(k)}}=c(k)$ with $c(k)=0$.

Now assume that $m>1$. Suppose $k \in \bar{I}\left(t^{\prime \prime}\right)$. Since $z$ is integral over $R$, we have that the coefficients of $f^{\prime}(Z)$ are in the integral closure $R_{k}^{\prime}$ of $R_{k}$ in $K^{\prime}$ (by Theorem 4, Section 3, Chapter V [47]), and $R_{k}^{\prime}$ is excellent since $R$ is. Further, $R_{k}^{\prime}$ is a regular local ring by 2 of Theorem 8.2. By the first part of 4 of Theorem 8.2, we have that $m$ is the highest power of $p$ which divides $d$. Applying Theorems 7.2 and 7.8 to $f^{\prime}(Z) \in R_{k}^{\prime}[Z]$, we can find $t^{\prime} \in \bar{I}\left(t^{\prime \prime}\right)$ such that for each $k \in \bar{I}\left(t^{\prime}\right)$ there exists $r_{k}^{\prime} \in R_{k}^{\prime}$ and nonnegative $a^{\prime}(k)$ and $c^{\prime}(k)$ such that $\operatorname{ord}_{S_{k}^{\prime}} f^{\prime}\left(r_{k}^{\prime}\right)=a^{\prime}(k), \operatorname{ord}_{R_{k}^{\prime} / x_{k}^{\prime} R_{k}^{\prime}} \frac{f^{\prime}\left(r_{k}^{\prime}\right)}{x_{k}^{\prime a^{\prime}(k)}}=$ $c^{\prime}(k),\left(a^{\prime}(k), c^{\prime}(k)\right) \not \equiv 0(m)$, and $c^{\prime}(k) \leq \frac{m}{p}$. Now by 4 of Theorem 8.2, there exists 
$r_{k} \in R_{k}$ and nonnegative integers $a(k)$ and $c(k)$ such that $\operatorname{ord}_{S_{k}} f\left(r_{k}+r_{k}^{*}\right)=a(k)$, $\operatorname{ord}_{R_{k} / x_{k} R_{k}} \frac{f\left(r_{k}+r_{k}^{*}\right)}{x_{k} a(k)}=c(k),(a(k), c(k)) \not \equiv 0(n)$, and $c(k) \leq \frac{n}{p}$ for all $r_{k}^{*} \in R_{k}$ with $\operatorname{ord}_{S_{k}} r_{k}^{*} \geq \frac{a(k)}{n}$.

Now we prove Theorem 8.1 in the essential case when $\nu$ is rational nondiscrete.

Let $R^{\prime \prime}=\hat{R}$ be the completion of $R$, and let $K^{\prime \prime}$ be the quotient field of $R^{\prime \prime}$. Let $L$ be a splitting field of $f(Z)$ over $K^{\prime \prime}$. Let $\nu^{\prime \prime}$ be the unique extension of $\nu$ to $K^{\prime \prime}$ which dominates $R^{\prime \prime}$. $\nu^{\prime \prime}$ is rational nondiscrete.

Let $L^{\prime}$ be the maximal separable extension of $K^{\prime \prime}$ in $L$, and let $\omega$ be an extension of $\nu^{\prime \prime}$ to $L^{\prime}$. Let $K^{\prime}$ be the splitting field of $\omega$ over $K^{\prime \prime}$, and let $\omega^{\prime}$ be the restriction of $\omega$ to $K^{\prime}$. Then $\omega$ and $\omega^{\prime}$ are rational, nondiscrete. $\omega^{\prime}$ does not split in $L^{\prime}$ by Proposition 1.46 [3]. Since $L$ is a purely inseparable extension of $L^{\prime}, \omega^{\prime}$ does not split in $L$. Let $Q_{b}$ be the localization of the integral closure $T_{b}^{\prime}$ of the $b$-th quadratic transform $R_{b}^{\prime \prime}$ of $R^{\prime \prime}$ along $\nu^{\prime \prime}$ in $L^{\prime}$ at the maximal ideal $T_{b}^{\prime} \cap M\left(V_{\omega}\right)$. By Lemma 14 [4], there exists a number $b_{0}$ such that for $b \geq b_{0}$, we have that $K^{\prime}$ is the splitting field of $Q_{b}$ over $R_{b}^{\prime \prime}$.

Suppose $b \geq b_{0}$ and let $R_{b}^{\prime}$ be the localization of the integral closure $T_{b}$ of $R_{b}^{\prime \prime}$ in $K^{\prime}$ at the maximal ideal $T_{b} \cap M\left(V_{\omega^{\prime}}\right)$. From Section 3 and Theorem 1.47 [3], we obtain that $R_{b}^{\prime}$ is an unramified extension of $R_{b}^{\prime \prime}$, so that $M\left(R_{b}\right) R_{b}^{\prime}=M\left(R_{b}^{\prime}\right)$, the residue field of $R_{b}^{\prime}$ is the algebraically closed field $\bar{k}, R_{b}^{\prime}$ is regular, $\hat{R}_{b}=\hat{R}_{b}^{\prime}=\hat{R}_{b}^{\prime \prime}$ and $R_{b}^{\prime} \rightarrow R_{b+1}^{\prime}$ is the quadratic transform of $R_{b}^{\prime}$ along $\omega^{\prime}$. We have thus reduced to the case where $R$ is complete and $\nu$ does not split in a splitting field $L$ of $f(Z)$. We make these assumptions for the remainder of the proof. In particular, $R_{k}$ is excellent for all $k$.

By Lemmas 8.4 and 8.5, it suffices to show that one of the following three conditions hold:

$$
Z^{n} \text { is an } R \text {-translate of } f(Z) \text { or }
$$

There exists $t^{\prime} \in \bar{I}$ and an $R_{t^{\prime}}$-translate $F(Z)=Z^{n}+F_{1} Z^{n-1}+\cdots+F_{n}$ of $f(Z)$ with $F_{1}, \ldots, F_{n} \in R_{t^{\prime}}$, and an integer $u$ with $0<u<n$ such that for all $k \in \bar{I}\left(t^{\prime}\right)$ we have that $F_{u}$ is an $R_{k}$-monomial in $x_{k}, \operatorname{ord}_{S_{k}} F_{j} \geq \frac{j}{u} \operatorname{ord}_{S_{k}} F_{u}$ for $1 \leq j \leq u$ and $\operatorname{ord}_{S_{k}} F_{j}>\frac{j}{u} \operatorname{ord}_{S_{k}} F_{u}$ for $u<j \leq n$ or

(40)

There exists $t^{\prime} \in \bar{I}$ such that for each $k \in \bar{I}\left(t^{\prime}\right)$ there exists an $R_{k}$-translate $f_{k}(Z)$ of $f(Z)$ and nonnegative integers $a(k)$ and $c(k)$ such that $f_{k}(Z)$ is of prenonsplittingtype relative to $\operatorname{ord}_{S_{k}}, \operatorname{ord}_{S_{k}} f_{k}(0)=a(k), \operatorname{ord}_{R_{k} / x_{k} R_{k}} \frac{f_{k}(0)}{x_{k}^{a(k)}}=c(k),(a(k), c(k)) \not \equiv 0(n)$, $c(k)=0$ if the degree of every nonconstant monic irreducible factor of $f(Z)$ in $K[Z]$ is nondivisible by $p$, and $c(k) \leq \frac{n}{p}$ if the degree of some nonconstant monic irreducible factor of $f(Z)$ in $K[Z]$ is divisible by $p$.

Let $g_{1}(Z), \ldots, g_{v}(Z)$ be the distinct nonconstant monic irreducible factors of $f(Z)$ in $K[Z]$. Then $g_{1}(Z), \ldots, g_{v}(Z)$ are in $R[Z]$ and there exists positive integers $e(1), \ldots, e(v)$ such that $f(Z)=f_{1}(Z) \cdots f_{v}(Z)$ where $f_{i}(Z)=g_{i}(Z)^{e(i)}$ for $1 \leq i \leq v$. Let $d(i)$ be the degree of $g_{i}(Z)$ in $Z$, and let $n(i)=d(i) e(i)$ for $1 \leq i \leq v$. Then $n=n(1)+\cdots+n(v)$. Let $z_{1}, \ldots, z_{v} \in L$ be such that $g_{i}\left(z_{i}\right)=0$ for $1 \leq i \leq v$.

First suppose that $d(i)=1$ for some $i$. Let $F(Z)=f\left(Z+z_{i}\right)$. Then $F(Z)$ is an $R$-translate of $f(Z)$ and $F(Z)=Z^{n}+F_{1} Z^{n-1}+\cdots+F_{n-1} Z$ where $F_{1}, \ldots, F_{n-1} \in R$. 
Let $V$ be the set of all integers $j$ with $1 \leq j \leq n-1$ such that $F_{j} \neq 0$. If $V$ is empty then $F(Z)=Z^{n}$ and (38) holds. Suppose that $V$ is nonempty. By embedded principalization of ideals in two dimensional regular local rings, there exists $t^{\prime} \in \bar{I}$ such that $F_{j}$ is an $R_{t^{\prime}}$-monomial in $x_{t^{\prime}}$ for all $j \in V$. Let $u$ be the greatest integer in $V$ such that

$$
\frac{1}{u} \operatorname{ord}_{S_{t^{\prime}}} F_{u}=\min _{j \in V} \frac{1}{j} \operatorname{ord}_{S_{t^{\prime}}} F_{j} .
$$

Then $0<u<n$ and for all $k \in \bar{I}\left(t^{\prime}\right)$ we have that $F_{u}$ is an $R_{k}$-monomial in $x_{k}$, $\operatorname{ord}_{S_{k}} F_{j} \geq \frac{j}{u} \operatorname{ord}_{S_{k}} F_{u}$ for $1 \leq j \leq u$, and $\operatorname{ord}_{S_{k}} F_{j}>\frac{j}{u} \operatorname{ord}_{S_{k}} F_{u}$ for $u<j \leq n$. Thus (39) holds.

We have reduced to the case where $d(i)>1$ for $1 \leq i \leq v$, which we will assume from now on. We have that $0 \neq D\left(g_{i}(Z), g_{j}(Z)\right) \in R$ whenever $1 \leq i \leq v, 1 \leq j \leq v$ and $i \neq j$. By embedded principalization of ideals in two dimensional regular local rings, there exists $t^{\prime \prime} \in \bar{I}$ such that $D\left(g_{i}(Z), g_{j}(Z)\right)$ is an $R_{k}$-monomial in $x_{k}$ whenever $k \in \bar{I}\left(t^{\prime \prime}\right), 1 \leq i \leq v, 1 \leq j \leq v$, and $i \neq j$. By 2 of Theorem 8.2, there exists $t \in \bar{I}\left(t^{\prime \prime}\right)$ such that $S_{k}$ is totally ramified in $L$ for all $k \in \bar{I}(t)$. For $1 \leq i \leq v$, by Theorem 8.6, there exists $t_{i} \in \bar{I}$ such that for each $k \in \bar{I}\left(t_{i}\right)$, there exists $r_{i k} \in R_{k}$ and nonnegative integers $a(i, k)$ and $c(i, k)$ such that $(a(i, k), c(i, k)) \not \equiv 0(n(i)), c(i, k)=0$ if $d(i) \not \equiv 0(p), c(i, k) \leq \frac{n(i)}{p}$ if $d(i) \equiv 0(p)$ and $\operatorname{ord}_{S_{k}} f_{i}\left(r_{i k}+r_{i k}^{*}\right)=a(i, k)$ and $\operatorname{ord}_{R_{k} / x_{k} R_{k}} \frac{f_{i}\left(r_{i k}+r_{i k}^{*}\right)}{x_{k}^{a(i, k)}}=c(i, k)$ for all $r_{i k}^{*} \in R_{k}$ with $\operatorname{ord}_{S_{k}} r_{i k}^{*} \geq \frac{a(i, k)}{n(i)}$.

Let $t^{\prime}=\max \left\{t, t_{1}, \ldots, t_{v}\right\}$. For any $k \in \bar{I}\left(t^{\prime}\right)$, by Lemma 8.3, there exists an $R_{k}$-translate $f_{k}(Z)$ of $f(Z)$ such that the conclusion (39) holds or there exist nonnegative integers $a(k)$ and $c(k)$ such that $f_{k}(Z)$ is of prenonsplitting type relative to $\operatorname{ord}_{S_{k}},(a(k), c(k)) \not \equiv 0(n), c(k) \leq c(1, k)+\cdots+c(v, k), \operatorname{ord}_{S_{k}} f_{k}(0)=a(k)$ and $\operatorname{ord}_{R_{k} / x_{k} R_{k}} \frac{f_{k}(0)}{x_{k}^{a(k)}}=c(k)$. It follows that $c(k)=0$ if $d(i) \not \equiv 0(p)$ for $1 \leq i \leq v$, and $c(k) \leq \frac{n}{p}$ if $d(i) \equiv 0(p)$ for some $i$, so that the conclusions of (40) hold.

9. Local uniformization and global resolution of singularities. In this section, suppose that $\bar{k}$ is an algebraically closed field of characteristic $p>0$, and $X$ is a nonsingular projective 3 -dimensional variety over $\bar{k}$; that is an integral projective scheme of dimension 3. Let $\bar{k}(X)$ be the function field of $X$. Suppose that $Y$ is a surface; that is a projective integral 2-dimensional subscheme of $X$.

Let $e$ be the largest multiplicity of a point on $Y$, and let

$$
\operatorname{Sing}_{e}(Y)=\left\{p \in Y \mid Y \text { has multiplicity } \nu_{p}(Y)=e \text { at } p\right\} .
$$

$\operatorname{Sing}_{e}(Y)$ is a closed subset of $Y$, which is a proper subset if $e>1$. $Y$ is nonsingular if $e=1$. We will suppose that $e>1$.

Suppose that $p \in \operatorname{Sing}_{e}(Y)$ is a closed point, $f \in \mathcal{O}_{X, p}$ is such that $f=0$ is a local equation of $Y$ at $p$, or $f \in \hat{\mathcal{O}}_{X, p}$ is such that $f=0$ is a formal local equation of $Y$ at $p$, and $(x, y, z)$ are regular parameters in $\mathcal{O}_{X, p}$ (or in $\left.\hat{\mathcal{O}}_{X, p}\right)$. There is an expansion

$$
f=\sum_{i+j+k \geq e} a_{i j k} x^{i} y^{j} z^{k}
$$

with $a_{i j k} \in \bar{k}$ in $\hat{\mathcal{O}}_{X, p}=\bar{k}[[x, y, z]]$. The leading form of $f$ with respect to $x, y, z$ is defined to be

$$
L(x, y, z)=\sum_{i+j+k=e} a_{i j k} x^{i} y^{j} z^{k} .
$$


The invariant $\tau(p)$ is the dimension of the smallest linear subspace $T$ of the $\bar{k}$-subspace spanned by $x, y$ and $z$ in $\bar{k}[x, y, z]$ such that $L \in \bar{k}[T]$. This dimension is independent of choice of regular parameters $(x, y, z)$ at $p$. If $x, y, z$ are regular parameters in $\mathcal{O}_{X, p}$, we will call the subvariety $M=V(T)$ of $\operatorname{spec}\left(\mathcal{O}_{X, p}\right)$ an approximate manifold to $Y$ at $p$. If $(x, y, z)$ are regular parameters in $\hat{\mathcal{O}}_{X, p}$, we call $M=V(T) \subset \operatorname{spec}\left(\hat{\mathcal{O}}_{X, p}\right)$ a (formal) approximate manifold to $Y$ at $p$. $M$ is dependent on our choice of regular parameters at $p$. Observe that $1 \leq \tau(q) \leq 3$. If there is a non-singular curve $C \subset \operatorname{Sing}_{e}(Y)$ such that $p \in C$, then $\tau(p) \leq 2$, and there exists an approximate manifold $M$ such that $M$ contains the germ of $C$ at $p$.

The following lemma is proven in Lemmas 7.4 and 7.5 of [17].

Lemma 9.1. Suppose that $Z \subset \operatorname{Sing}_{e}(Y)$ is a nonsingular subvariety of $X$ (a point or a curve), $\Phi_{1}: X_{1} \rightarrow X$ is the blow up of $Z, Y_{1}$ is the strict transform of $Y$ on $X_{1}, p \in Z, M_{p}$ is an approximate manifold to $Y$ at $p$ containing the germ of $Z$ at $p$, and $q \in \Phi_{1}^{-1}(p)$. Then

1. $\nu_{q}\left(Y_{1}\right) \leq e$.

2. $\nu_{q}\left(Y_{1}\right)=e$ implies $q$ is on the strict transform $M_{p}^{\prime}$ of $M_{p}$ and $\tau(p) \leq \tau(q)$.

3. Suppose that $\nu_{q}\left(Y_{1}\right)=e$ and $\tau(p)=\tau(q)$. Then there exists an approximate manifold $M_{q}$ to $Y_{1}$ at $q$ such that $M_{q} \cap \Phi_{1}^{-1}(p)=M_{p}^{\prime} \cap \Phi_{1}^{-1}(p)$ where $M_{p}^{\prime}$ is the strict transform of $M_{p}$ on $X_{1}$.

Definition 9.2. A projective morphism $\Phi: X_{1} \rightarrow X$ is a permissible blow up (for $Y$ ) if $\Phi$ is the blow up of a nonsingular curve or point which is contained in Sing $_{e}(Y) . \Phi$ is a strictly permissible blow up if $\Phi$ is a permissible blow up of an irreducible component of $\operatorname{Sing}_{e}(Y)$.

DeFINITION 9.3. $Y$ is prepared if $\operatorname{Sing}_{e}(Y)$ is a union of points and nonsingular curves such that two curves intersect in at most one point, at most two curves of $\operatorname{Sing}_{e}(Y)$ pass through any given point of $Y$, and if two curves $C_{1}, C_{2}$ pass through a point $p$ of $Y$, then they have normal crossings there (there exist regular parameters $x, y, z$ in $\mathcal{O}_{X, p}$ such that $x=z=0$ are local equations of $C_{1}$ at $p$ and $y=z=0$ are local equations of $C_{2}$ at $p$ ).

The following theorems 9.4 and 9.5 are proven in Theorem 7.7 [17].

By a sequence of permissible blow ups $X_{m} \rightarrow \cdots \rightarrow X_{1} \rightarrow X_{0}=X$, we mean a sequence of morphisms such that each $\Phi_{i}: X_{i} \rightarrow X_{i-1}$ is permissible for $Y_{i-1}$, where $Y_{i}$ is the strict transform of $Y$ on $X_{i}$.

THEOREM 9.4. There exists a sequence of permissible blow ups

$$
X_{m} \rightarrow X_{m-1} \rightarrow \cdots \rightarrow X_{0}=X
$$

such that $Y_{m}$ is prepared. Further, if

$$
X_{n} \rightarrow X_{n-1} \rightarrow \cdots \rightarrow X_{m}
$$

is any sequence of permissible blow ups, then $Y_{n}$ is prepared.

Theorem 9.5. Suppose that $Y$ is prepared. Suppose that

$$
\cdots \rightarrow X_{m} \rightarrow X_{m-1} \rightarrow \cdots \rightarrow X_{0}=X
$$


is a sequence of permissible blow ups of curves. Then the sequence is finite. In particular, if (41) is any maximal sequence, then $\operatorname{Sing}_{e}\left(Y_{m}\right)$ is a finite union of points.

Lemma 9.6. Let $S$ be an $n$-dimensional regular local ring with a regular system of parameters $x_{1}, \ldots, x_{n}$. Let $f(Z) \in S[Z]$ be monic of degree $e>0$. Let $r \in S$ and let $s=t x_{1}^{a_{1}} \cdots x_{n}^{a_{n}}$ where $t$ is a unit in $S$ and $a_{1}, \ldots, a_{n}$ are nonnegative integers. Let $g(Z)=s^{-e} f(s Z+r)$. Assume that $g(Z) \in S[Z]$ and $0<\operatorname{ord}_{S} g(Z)<e$.

Let $r^{\prime} \in S$ and let $s^{\prime}=t^{\prime} x_{1}^{b_{1}} \cdots x_{n}^{b_{n}}$ where $t^{\prime}$ is a unit in $S$ and $b_{1}, \ldots, b_{n}$ are nonnegative integers. Let $g^{\prime}(Z)=s^{\prime-e} f\left(s^{\prime} Z+r^{\prime}\right), r^{*}=\frac{r^{\prime}-r}{s^{\prime}}, c_{i}=a_{i}-b_{i}$ for $1 \leq i \leq n, t^{*}=\frac{t}{t^{\prime}}$ and $s^{*}=t^{*} x_{1}^{c_{1}} \cdots x_{n}^{c_{n}}$, so that $g(Z)=s^{*-e} g^{\prime}\left(s^{*} Z-r^{*}\right)$. Assume that $g^{\prime}(Z) \in S[Z]$. Then $r^{*} \in S$ and $c_{i} \geq 0$ for $1 \leq i \leq n$.

This is proven in (4.10) of [7].

Theorem 9.7 shows that after a sequence of blow ups of points along a valuation, we reduce to a good point; that is, a point from which a reduction of multiplicity can be obtained by only blowing up curves.

THEOREM 9.7. Suppose that $Y$ is prepared and $\nu$ is a 0 -dimensional valuation of $\bar{k}(X)$. Then there exists a sequence of permissible blow ups

$$
X_{m} \rightarrow X_{m-1} \rightarrow \cdots \rightarrow X_{0}=X
$$

such that the center of $\nu$ on $X_{m}$ is not in $\operatorname{Sing}_{e}\left(Y_{m}\right)$.

Proof. Let

$$
\cdots \rightarrow X_{m} \rightarrow X_{m-1} \rightarrow \cdots \rightarrow X_{0}=X
$$

be the possibly infinite sequence of permissible blow ups obtained by blowing up the center $p_{n}$ of $\nu$ on $X_{n}$, if $p_{n} \in \operatorname{Sing}_{r}\left(Y_{n}\right)$ (the center exists and is uniquely determined by the valuative crieterion of perperness, and is a closed point since $\nu$ is 0 -dimensional). If this sequence is finite then we have constructed the desired sequence (42). Suppose that the sequence is not finite. Let $R_{i}=\mathcal{O}_{X_{n}, p_{n}}$. Then we have an infinite sequence of quadratic transforms of two dimensional regular local rings

$$
R_{0} \rightarrow R_{1} \rightarrow \cdots \rightarrow R_{n} \rightarrow \cdots
$$

which are dominated by $\nu$. Let $\bar{f} \in R_{0}$ be such that $\bar{f}=0$ is a local equation of $Y$ at $p$. Since $\bar{k}$ is infinite and by the Weierstrass preparation theorem, there exist regular parameters $x, y, z$ in $R$, a unit $\lambda \in \hat{R}$ and $a_{i} \in \bar{k}[[x, y]]$ such that $\bar{f}=$ $\lambda\left(z^{e}+a_{1} z^{e-1}+\cdots+a_{e}\right) \in \bar{k}[[x, y, z]]=\hat{R}$. Let $f(z)=z^{e}+a_{1} z^{e-1}+\cdots+a_{e}$. Then $f=0$ is a formal local equation of $Y$ at $p$. Let $S=\bar{k}[[x, y]] . S[z]$ is isomorphic to a polynomial ring $S[Z]$ in a variable $Z$. We have that $f(Z)$ is reduced in $S[Z]$ since $\bar{f}$ is irreducible in $R$, and $R$ is excellent. By Lemma 9.1, for all $i$, we have regular parameters $x_{i+1}, y_{i+1}, z_{i+1}$ in $R_{i+1}$ such that

$$
x_{i}=x_{i+1}, y_{i}=x_{i+1}\left(y_{i+1}+\alpha_{i+1}\right), z_{i}=x_{i+1}\left(z_{i+1}+\lambda_{i+1}\right) \text { with } \alpha_{i+1} \in \bar{k}, \lambda_{i+1} \in \bar{k}\left[x_{i}, y_{i}\right],
$$

or

$$
x_{i}=x_{i+1} y_{i+1}, y_{i}=y_{i+1}, z_{i}=y_{i+1}\left(z_{i+1}+\lambda_{i+1}\right) \text { with } \lambda_{i+1} \in \bar{k}\left[x_{i}, y_{i}\right] .
$$

Let $S_{i}=S_{0}\left[x_{i}, y_{i}\right]_{\left(x_{i}, y_{i}\right)}$ which is a regular local ring of dimension two. 
Let $\nu^{\prime}$ be an extension of $\nu$ to the quotient field of $\hat{R}$ which dominates $\hat{R}$. When we restrict to the quotient field of $K$ of $S$, we get a valuation $\bar{\nu}$. The infinite sequence of quadratic transformations

$$
S_{0} \rightarrow S_{1} \rightarrow \cdots \rightarrow S_{n} \rightarrow \cdots
$$

is dominated by $\bar{\nu}$. Since $f$ is reduced, by Theorem 8.1, after possibly making a change of regular parameters in $S$, we have that

There exists $n>0, r \in \hat{S}_{n}$ and nonnegative integers $u$ and $v$ such that after setting $g(Z)=\left(x_{n}^{u} y_{n}^{v}\right)^{-e} f\left(x_{n}^{u} y_{n}^{v} Z+r\right)$ we have that $g(Z) \in S_{n}[Z]$ and $0<\operatorname{ord}_{S_{n}} g(Z)<e$.

We have an expression $z_{n}=s^{\prime} z+r^{\prime}$, where $s^{\prime}=t^{\prime} x_{n}^{a^{\prime}} y_{n}^{b^{\prime}}$ with $t^{\prime} \in S_{n}$ a unit and $r^{\prime} \in S_{n}$. Set $g^{\prime}(Z)=s^{\prime-e} f\left(s^{\prime} Z+r^{\prime}\right) . g^{\prime}(Z) \in S_{n}[Z]$ and $g^{\prime}\left(z_{n}\right)=0$ is a formal local equation of $Y_{n}$ at $p_{n}$.

Set $r^{*}=\frac{r^{\prime}-r}{s^{\prime}}, a=u-a^{\prime}, b=v-b^{\prime}$ and $s^{*}=\left(t^{\prime}\right)^{-1} x_{n}^{a} y_{n}^{b}$. By Lemma 9.6, we have that $r^{*} \in \hat{S}_{n}, a, b \in \mathbb{N}$ and $g(Z)=s^{*-e} g^{\prime}\left(s^{*} Z-r^{*}\right)$. Now $g^{\prime}\left(z_{n}\right) \in M\left(\hat{R}_{n}\right)^{e}$ implies $g^{\prime}(Z)-Z^{e} \in M\left(\hat{S}_{n}\right)[Z]$. Now $g^{\prime}\left(s^{*} z_{n}-r^{*}\right)=s^{* e} g\left(z_{n}\right) \in M\left(\hat{R}_{n}\right)$ implies $r^{*} \in M\left(\hat{R}_{n}\right)$. Thus $r^{*} \in M\left(\hat{S}_{n}\right)$. Set $z^{*}=z_{n}+r^{*}$. Then $x_{n}, y_{n}, z^{*}$ is a regular system of parameters in $\hat{R}_{n}$. Let $g^{*}(Z)=\left(t^{\prime}\right)^{-e} g\left(t^{\prime} Z\right)$. Then $g^{*}(Z) \in \hat{S}_{n}[Z]$ and $\operatorname{ord}_{\hat{S}_{n}} g^{*}(Z)=\operatorname{ord}_{\hat{S}_{n}} g(Z)$. Hence

$$
g^{*}(Z)=Z^{e}+g_{1} Z^{e-1}+\cdots+g_{e}
$$

where $g_{1}, \ldots, g_{e}$ are elements in $\hat{S}_{n}$ such that $g_{e} \in M\left(\hat{S}_{n}\right)$ and $g_{j^{\prime}} \notin M\left(\hat{S}_{n}\right)^{j^{\prime}}$ for some $j^{\prime}$ with $1 \leq j^{\prime} \leq e$. Let $w=g^{\prime}\left(z_{n}\right)$. Then $w=0$ is a formal local equation of $Y_{n}$ at $p_{n}$. Also $g^{\prime}\left(z_{n}\right)=x_{n}^{a e} y_{n}^{b e} g^{*}\left(x_{n}^{-a} y_{n}^{-b} z^{*}\right)$, and hence

$$
w=\left(z^{*}\right)^{e}+\sum_{j=1}^{e} g_{j} x_{n}^{a j} y_{n}^{b j}\left(z^{*}\right)^{e-j}
$$

with $g_{e} \in M\left(\hat{S}_{n}\right)$, and $\operatorname{ord}_{\hat{S}_{n}} g_{j^{\prime}}<j^{\prime}$ for some $j^{\prime}$ with $1 \leq j^{\prime} \leq e$. Since $p_{n} \in \operatorname{Sing}_{r}\left(Y_{n}\right)$, we must have that $a+b \geq 1$. Let $L$ be the leading form of $w$ in $\bar{k}\left[\left[x_{n}, y_{n}, z^{*}\right]\right]$. Since $g_{e} \in M\left(\hat{R}_{n}\right)$, we have that

$$
z^{*} \text { divides } L \text {. }
$$

Without loss of generality, we can assume that $a \geq 1$. Let $I=x_{n} \hat{\mathcal{O}}_{X_{n}, p_{n}}+$ $z^{*} \hat{\mathcal{O}}_{X_{n}, p_{n}}$. Then $w \in I^{e}$. Since $w=0$ is a formal local equation of $Y_{n}$ at $p_{n}$ (and $\mathcal{O}_{X_{n}, p_{n}}$ is excellent), there exists a permissible curve $C$ in $X_{n}$, with ideal sheaf $\mathcal{I}_{C}$ on $X_{n}$ such that $\widehat{\mathcal{I}_{C, p_{n}}}=I$. Let $\Psi_{n+1}: \tilde{X}_{n+1} \rightarrow X_{n}$ be the blow up of $C$. Suppose that there exists a point $q \in \operatorname{Sing}_{e}\left(\tilde{Y}_{n+1}\right)$ such that $\Psi_{n+1}(q)=p_{n}$. Let $T=\widehat{\mathcal{O}_{\tilde{X}_{n+1}, q}}$. Then by Lemma 9.1 and (45), we have that $T$ has regular parameters $\tilde{x}, \tilde{y}, \tilde{z}$ where $x_{n}=\tilde{x}$, $y_{n}=\tilde{y}$ and $z^{*}=\tilde{x} \tilde{z}$. Substituting into (44), we obtain a formal local equation of $\tilde{Y}_{n+1}$ of the same form as (44), but with a reduction of $a+b$ by 1 .

Repeating this process, we construct a sequence of blow ups of permissible curves which terminates with a drop in the multiplicity of the strict transform of $Y$ at the center of $\nu$ after at most $a+b$ blow ups. 
Corollary 9.8. (Local Uniformization) Suppose that $Y \subset X$ is a surface contained in a nonsingular 3-fold $X$ over an algebraically closed field $\bar{k}$ of characteristic $p>0$ and $\nu$ is a valuation of the function field $\bar{k}(X)$ of $X$. Then there exists a sequence of blow ups of points and nonsingular curves

$$
X_{m} \rightarrow X_{m-1} \rightarrow \cdots \rightarrow X_{0}=X
$$

such that if the center $p$ of $\nu$ on $X_{m}$ is on the strict transform $Y_{m}$ of $Y$, then $p$ is a nonsingular point of $Y_{m}$.

Proof. We can (if necessary) compose $\nu$ with a 0 -dimensional valuation of the residue field of $V_{\nu}$ to obtain a 0 -dimensional valuation $\bar{\nu}$ of $\bar{k}(X)$ (Section 16, Chapter VI [47] or Section 10 [3]). Then the corollary for $\bar{\nu}$ follows from induction on $e$ from a local version of Theorems 9.4 and 9.5 and from Theorem 9.7. Since $\bar{\nu}$ is a specialization of $\nu$, the local ring of the center of $\nu$ on $X_{m}$ is a localization of the local ring of the center of $\bar{\nu}$ on $X_{m}$. Thus the corollary hold for $\nu$ on $X_{m}$ also.

Lemma 9.9. Suppose that $Y$ is prepared and $p \in \operatorname{Sing}_{e}(Y)$. Suppose that $C_{1}, C_{2}$ are two curves in $\operatorname{Sing}_{e}(Y)$ containing $p$. Let $\Phi_{1}: X_{1} \rightarrow X$ be the blow up of $C_{1}$ and let $\Phi_{2}: X_{2} \rightarrow X_{1}$ be the blow up of the strict transform of $C_{2}$. Suppose that there exists $p_{2} \in \operatorname{Sing}_{e}\left(Y_{2}\right)$ such that $\Phi_{1} \circ \Phi_{2}\left(p_{2}\right)=p$ (so that $p_{2}$ is the unique such point by Lemma 9.1). Let $\nu$ be a 0-dimensional valuation of $\bar{k}(X)$ whose center on $X_{2}$ is $p_{2}$.

Let $\Psi_{1}: \bar{X}_{1} \rightarrow X$ be the blow up of $C_{2}$ and let $\Psi_{2}: \bar{X}_{2} \rightarrow \bar{X}_{1}$ be the blow up of the strict transform of $C_{1}$. Let $q_{2}$ be the center of $\nu$ on $\bar{X}_{2}$.

Then $\mathcal{O}_{\bar{X}_{2}, q_{2}}=\mathcal{O}_{X_{2}, p_{2}}$ and $q_{2} \in \operatorname{Sing}_{e}\left(\bar{X}_{2}\right)$

Proof. There exist regular parameters $x, y, z$ in $\mathcal{O}_{X, p}$ such that $\mathcal{I}_{C_{1}, p}=(x, z)$ and $\mathcal{I}_{C_{2}, p}=(y, z)$. Let $f \in \mathcal{O}_{X, p}$ be such that $f=0$ is a local equation of $Y$ at $p$. $f \in(x, z)^{e} \cap(y, z)^{e}$ so that the leading form of $f$ in $\hat{\mathcal{O}}_{X, p}=\bar{k}[[x, y, z]]$ is a constant time $z^{e}$.

Let $p_{1} \in \operatorname{Sing}_{e}\left(X_{1}\right)$ be the point on the strict transform of $C_{2}$. We have by Lemma 9.1 that $\mathcal{O}_{X_{1}, p_{1}}$ has regular parameters $x_{1}, y_{1}, z_{1}$ where $x=x_{1}, y=y_{1}$ and $z=x_{1} z_{1}$. Let $f_{1}=\frac{f}{x^{e}} . f_{1}=0$ is a local equation of $Y_{2}$ at $p_{1}$. By Lemma 9.1, the leading form $L_{1}$ of $f_{1}$ has the form $L_{1}=\left(z_{1}-\alpha y_{1}\right)^{e}$ times a constant for some $\alpha \in \bar{k}$.

Thus $\mathcal{O}_{X_{2}, p_{2}}$ has regular parameters $x_{2}, y_{2}, z_{2}$ where $x_{1}=x_{2}, y_{1}=y_{2}$ and $z_{1}=$ $y_{2}\left(z_{2}+\alpha\right)$. Set $\bar{z}=z-\alpha x y$. Then $x, y, \bar{z}$ are regular parameters in $\mathcal{O}_{X, p}$ and $x=x_{2}, y=y_{2}, \bar{z}=x_{2} y_{2} z_{2}$. Thus there exists a point $\bar{q} \in\left(\Psi_{1} \Psi_{2}\right)^{-1}(p)$ such that $\mathcal{O}_{\bar{X}_{2}, \bar{q}}=\mathcal{O}_{X_{2}, p_{2}}$, and thus $\bar{q}=q_{2} \in \operatorname{Sing}_{e}\left(\bar{Y}_{2}\right)$.

The following lemma, which follows from (3.10.6) [7], is proved in a similar way.

Lemma 9.10. Suppose that $Y$ is prepared. Suppose that $C$ is a curve in $\operatorname{Sing}_{e}(Y)$ and $p \in C$ is a point. Let $\Phi_{1}: X_{1} \rightarrow X$ be the blow up of $C$ and $\Phi_{2}: X_{2} \rightarrow X_{1}$ be a permissible blow up of a point $p_{1}$ in $\Phi_{1}^{-1}(p)$. Suppose that $p_{2} \in \operatorname{Sing}_{e}\left(Y_{2}\right)$ is a point such that $\Phi_{2} \Phi_{1}\left(p_{2}\right)=p$. Let $\nu$ be a 0-dimensional valuation of $\bar{k}(X)$ whose center on $\mathrm{X}_{2}$ is $p_{2}$.

Let $\Psi_{1}: \bar{X}_{1} \rightarrow X$ be the blow up of $p$. Then there is a unique curve $\bar{C}$ in $\operatorname{Sing}_{e}\left(\bar{Y}_{1}\right)$ such that $\Psi_{1}(\bar{C})=p$. Let $\Psi_{2}: \bar{X}_{2} \rightarrow \bar{X}_{1}$ be the blow up of $\bar{C}$, and let $\Psi_{3}: \bar{X}_{3} \rightarrow \bar{X}_{2}$ be the blow up of the strict transform of $C$.

Let $q_{3}$ be the center of $\nu$ on $X_{3}$. Then $\mathcal{O}_{\bar{X}_{3}, q_{3}}=\mathcal{O}_{X_{2}, p_{2}}$, and $q_{3} \in \operatorname{Sing}_{e}\left(\bar{X}_{3}\right)$.

Lemma 9.11. Suppose that $Y$ is prepared. Let

$$
X_{n} \rightarrow X_{n-1} \rightarrow \cdots \rightarrow X_{1} \rightarrow X_{0}=X
$$


be a sequence of strictly permissible blow ups, such that $\Phi_{i}: X_{i} \rightarrow X_{i-1}$ is the blow up of a curve for $i<n$ and $\Phi_{n}: X_{n} \rightarrow X_{n-1}$ is the blow up of a point $p_{n-1}$.

Let $p_{n} \in \Phi_{n}^{-1}\left(p_{n-1}\right) \cap \operatorname{Sing}_{e}\left(Y_{n}\right)$, and let $\nu$ be a 0 -dimensional valuation of $\bar{k}(X)$ whose center on $X_{n}$ is $p_{n}$. Suppose that $\Psi: Z_{1} \rightarrow X$ is a permissible blow up. Then there exists a sequence of strictly permissible blow ups

$$
Z_{m} \rightarrow \cdots \rightarrow Z_{1}
$$

such that if $q_{m}$ is the center of $\nu$ on $Z_{m}$, then $\mathcal{O}_{Z_{m}, q_{m}}=\mathcal{O}_{X_{n}, p_{n}}$.

Proof. We may assume that each $\Phi_{i}: X_{i} \rightarrow X_{i-1}$ is the blow up of a subvariety containing the center $p_{i}$ of $\nu$ on $X_{i}$. The lemma is trivial if $p=p_{0}$ is not in the subvariety of $X$ blown up by $\Psi$, so we will assume that $p$ is in this subvariety. We prove the lemma by induction on $n$. Let $p_{i}$ be the center of $\nu$ on $X_{i}$ for $i \leq m$, and let $q_{1}$ be the center of $\nu$ on $Z_{1}$. If $n=1$, we must have that $\operatorname{Sing}_{e}(X)$ is a finite union of points. Hence $Z_{1} \rightarrow X$ must be the blow up of $p$, so that $Z_{1} \cong X_{1}$.

Suppose that $n=2$. Then there is a unique curve $C$ in $\operatorname{Sing}_{e}(X)$ containing $p$, $X_{1} \rightarrow X$ is the blow up of this curve and $X_{2} \rightarrow X_{1}$ is the blow up of a point on the exceptional divisor over $C$. Thus either $Z_{1}=X_{1}$, in which case the conclusions of the lemma are trivially true, or $Z_{1} \rightarrow X_{1}$ is the blow up of $p$. In this case, the conclusions of the lemma follow from Lemma 9.10.

Now assume that $n \geq 3$ and the lemma is true for sequences (47) of length $n-1$. Since $n>1, p$ must lie on a curve $C_{1}$ in $\operatorname{Sing}_{e}(Y)$ such that $X_{1} \rightarrow X$ is the blow up of $C_{1}$. If $Z_{1} \rightarrow X$ is the blow up of $C_{1}$ then $Z=X_{1}$. The remaining cases are when $Z \rightarrow X$ is a blow up of another curve $C_{2}$ in $\operatorname{Sing}_{e}(Y)$ containing $p$ and when $Z \rightarrow X$ is the blow up of $p$. We will consider these cases separately.

Suppose that $Z_{1} \rightarrow X$ is the blow up of another curve $C_{2}$ in $\operatorname{Sing}_{e}(Y)$ containing $p$. Let $\tilde{C}_{2}$ be the strict transform of $C_{2}$ on $X_{1}$. Since $\tilde{C}_{2}$ is contained in $\operatorname{Sing}_{e}\left(Y_{1}\right)$ and $p_{1}$ must be the only point in $\Psi_{1}^{-1}(p) \cap \operatorname{Sing}_{r}\left(Y_{1}\right)$ (by Lemma 9.1), we have that $p_{1} \in \tilde{C}_{2}$. Let $X_{1}^{\prime} \rightarrow X_{1}$ be the blow up of $\tilde{C}_{2}$, and let $q_{1}^{\prime}$ be the center of $\nu$ on $X_{1}^{\prime}$. By induction on $n$, there exists a strict permissible sequence $X_{m}^{\prime} \rightarrow \cdots \rightarrow X_{1}^{\prime}$ such that if $q_{i}^{\prime}$ is the center of $\nu$ on $X_{i}^{\prime}$, then $\mathcal{O}_{X_{m}^{\prime}, q_{m}^{\prime}}=\mathcal{O}_{X_{n}, p_{n}}$. Let $\tilde{C}_{1}$ be the strict transform of $C_{1}$ on $Z_{1}$, and let $Z_{2} \rightarrow Z_{1}$ be the (strictly permissible) blow up of $\tilde{C}_{2}$. Now by Lemma 9.9, The local ring of the center of $\nu$ on $Z_{2}$ is equal to the local ring of the center of $\nu$ on $X_{1}^{\prime}$, and thus we may splice the permissible sequences together to obtain the conclusions of the lemma in this case.

Now suppose that $Z_{1} \rightarrow X$ is the blow up $p$. Let $X_{1}^{\prime} \rightarrow X_{1}$ be the blow up of $p_{1}$. By induction on $n$, there exists a strictly permissible sequence $X_{m}^{\prime} \rightarrow \cdots \rightarrow X_{1}^{\prime}$ such that if $q_{i}^{\prime}$ is the center of $\nu$ on $X_{i}^{\prime}$, then $\mathcal{O}_{X_{m}^{\prime}, q_{m}^{\prime}}=\mathcal{O}_{X_{n}, p_{n}}$.

By Lemma 9.10, applied to the permissible sequence $X_{1}^{\prime} \rightarrow X_{1} \rightarrow X$, there exists a permissible sequence $Z_{3} \rightarrow Z_{2} \rightarrow Z_{1}$ such that $Z_{3} \rightarrow Z_{2}$ and $Z_{2} \rightarrow Z_{1}$ are strictly permissible, and if $q_{3}$ is the center of $\nu$ on $Z_{3}$, then $\mathcal{O}_{Z_{3}, q_{3}}=\mathcal{O}_{X_{1}^{\prime}, q_{1}^{\prime}}$. Now we may splice the permissible sequences together to obtain the conclusions of the lemma.

TheOREm 9.12. (The Theorem of Beppo Levi) Suppose that $Y$ is prepared. Let

$$
\cdots \rightarrow X_{n} \rightarrow X_{n-1} \rightarrow \cdots \rightarrow X_{1} \rightarrow X_{0}=X
$$

be any sequence of strictly permissible blow ups. Then the sequence terminates after a finite number of blow ups. In particular, this algorithm leads to a reduction $\operatorname{Sing}_{e}\left(Y_{n}\right)=\emptyset$ if the sequence has maximal length. 
Proof. Suppose that the sequence (48) has infinite length. Since the center of each blow up $\Phi_{i}: X_{i} \rightarrow X_{i-1}$ in the sequence is an irreducible component of $\operatorname{Sing}_{e}\left(Y_{i}\right)$, and for each $i, \operatorname{Sing}_{e}\left(Y_{i}\right)$ has only a finite number of irreducible components, there exists an infinite sequence of points $\left\{p_{i}\right\}$ such that $p_{i} \in X_{i}, \Phi_{i}\left(p_{i}\right)=p_{i-1}$ for all $i$ and infinitely many of the $\Phi_{i}$ are not isomorphisms at $p_{i}$. We may then replace (48) with the infinite sequence consisting only of blow ups of points and curves in (48) which contain $p_{i}$. We then obtain an infinite sequence

$$
R=R_{0} \rightarrow R_{1} \rightarrow \cdots \rightarrow R_{i} \rightarrow \cdots
$$

of monoidal transforms of 3-dimensional regular local rings $R_{i}=\mathcal{O}_{X_{i}, p_{i}}$. Let $I_{i}=$ $\mathcal{I}_{Y_{i}, p_{i}}$. We have that $I_{i}$ is a principal ideal of multiplicity $e$ for all $i$.

Let $D=\cup_{i=0}^{\infty} R_{i} . \quad D$ is a (possibly non noetherian) quasi local domain. By Theorem 5, Section 4, Chapter VI [47], there exists a valuation $\nu$ of $\bar{k}(X)$ such that $\nu$ dominates $D$, and hence dominates all of the $R_{i}$ in the sequence (49). After possibly composing $\nu$ with a valuation of the residue field of $V_{\nu}$, we may assume that $\nu$ is 0-dimensional (Section 16, Chapter VI [47] or Section 10 [3]).

Suppose that

$$
\bar{X}_{m} \rightarrow \cdots \rightarrow \bar{X}_{0}=X
$$

is a sequence of permissible blow ups. Let $q_{i}$ be the center of $\nu$ on $\bar{X}_{i}$ for $1 \leq i \leq m$.

We will prove that for all $i$ with $1 \leq i \leq m$, there exist positive integers $\sigma(i)$ and $\tau(i)$, and a sequence of strictly permissible blow ups

$$
Z_{\tau(i)}^{i} \rightarrow \cdots \rightarrow Z_{i+1}^{i} \rightarrow \bar{X}_{i}
$$

such that if the center of $\nu$ on $Z_{\tau(i)}^{i}$ is $q_{\tau(i)}^{i}$, then $\mathcal{O}_{Z_{\tau(i), q_{\tau(i)}^{i}}}=\mathcal{O}_{X_{\sigma(i)}, p_{\sigma(i)}}$.

We prove this assertion, constructing sequences (50), by induction on $m$. We first prove the case $m=1$. Since (49) is infinite, there exists by Theorem 9.5, an $n_{1} \geq 1$ such that $X_{n_{1}} \rightarrow X_{n_{1}-1}$ is the blow up of the point $p_{n_{1}-1}$, and $X_{i} \rightarrow X_{i-1}$ is the blow up of a curve for $i<n_{1}$. By Lemma 9.11, we can construct a sequence (50) for $i=1$, with $\sigma(1)=n_{1}$.

Now suppose that the assertion is true for $m-1$, so that we have constructed a sequence (50) with $i=m-1$. We may thus construct an infinite sequence of permissible blow ups

$$
\cdots \rightarrow Z_{j}^{m-1} \rightarrow Z_{\tau(m-1)+1}^{m-1} \rightarrow Z_{\tau(m-1)}^{m-1} \rightarrow \cdots \rightarrow Z_{m}^{m-1} \rightarrow \bar{X}_{m-1}
$$

by performing the corresponding blowups of

$$
\cdots \rightarrow X_{n} \rightarrow \cdots \rightarrow X_{\sigma(m-1)}
$$

above $Z_{\tau(m-1)}^{m-1}$, so that if $q_{j}^{m-1}$ is the center of $\nu$ on $Z_{j}^{m-1}$, then

$$
\mathcal{O}_{Z_{j}^{m-1}, q_{j}^{m-1}} \cong \mathcal{O}_{X_{\sigma(m-1)-\tau(m-1)+j}, p_{\sigma(m-1)-\tau(m-1)+j}}
$$

for $j \geq \tau(m-1)$. Now by Theorem 9.5 , there exists an $n \geq m-1$ such that $Z_{n+1}^{m-1} \rightarrow Z_{n}^{m-1}$ is the blow up of $q_{n}^{m-1}$, and $Z_{i+1}^{m-1} \rightarrow Z_{i}^{m-1}$ is the blow up of a curve for $m-1 \leq i<n$. By Lemma 9.11, we can construct a sequence (50) for $i=m$. 
We conclude that the center $q_{m}$ of $\nu$ on $X_{m}$ must be in $\operatorname{Sing}_{e}\left(\bar{Y}_{m}\right)$. Applying this result to the sequence (42) of the conclusions of Theorem 9.7, we obtain a contradiction, as $q_{m} \notin \operatorname{Sing}_{e}\left(\bar{Y}_{m}\right)$.

Corollary 9.13. (Resolution of Surface Singularities) Suppose that $Y \subset X$ is a surface. Then there exists a sequence of blow ups of points and nonsingular curves, contained in the singular locus of the strict transform of $Y$,

$$
X_{n} \rightarrow X_{n-1} \rightarrow \cdots \rightarrow X_{1} \rightarrow X_{0}=X
$$

such that the strict transform $Y_{n}$ of $Y$ on $X_{n}$ is nonsingular.

Our proof of Theorem 9.12 extends without difficulty to the case where $Y$ is a reduced, but not necessarily integral, surface. The analysis in Sections $5-8$ and 11 of [18] reduces the proof of the following theorems to the Theorem of Beppo Levi for reduced surfaces. This part of the proof involves no essential differences between characteristic zero and characteristic $p>0$. in Sections 9 and 10 of [18], we use Hironaka's resolution algorithm to prove the Theorem of Beppo Levi for reduced surfaces.

TheOREM 9.14. (Embedded Resolution of Surface Singularities) Suppose that X is a nonsingular 3-dimensional variety over an algebraically closed field $\bar{k}$ of characteristic $p>0, Y$ is a reduced surface in $X$ (a pure 2-dimensional reduced closed subscheme) and $E$ is a simple normal crossings divisor on $X$. Then there exists a sequence of morphisms

$$
\pi: X_{n} \rightarrow X_{n-1} \rightarrow \cdots \rightarrow X_{1} \rightarrow X
$$

such that the strict transform $Y_{n}$ of $Y$ on $X_{n}$ is nonsingular, and the divisor $\pi^{*}(Y+E)$ is a simple normal crossings divisor on $X_{n}$. Further, each $X_{i} \rightarrow X_{i-1}$ is the blow up of a point or nonsingular curve in the locus in $X_{i-1}$ where the preimage of $Y+E$ is not a simple normal crossings divisor.

THEOREM 9.15. (Principalization of Ideals) Suppose that $X$ is a nonsingular 3-dimensional variety over an algebraically closed field $\bar{k}$ of characteristic $p>0$. Suppose that $\mathcal{I}$ is a nonzero ideal sheaf on $X$. Then there exists a sequence of morphisms

$$
X_{n} \rightarrow X_{n-1} \rightarrow \cdots \rightarrow X_{1} \rightarrow X
$$

such that $\mathcal{I O}_{X_{n}}$ is invertible. Further, each $X_{i} \rightarrow X_{i-1}$ is the blow up of a point or nonsingular curve in the locus in $X_{i-1}$ where $\mathcal{I O}_{X_{i-1}}$ is not invertible.

\section{REFERENCES}

[1] S. Abhyankar, Local Uniformization on Algebraic Surfaces Over Ground Fields of Characteristic $p \neq 0$, Annals of Math., 63 (1956), pp. 491-256.

[2] S. Abhyankar, Uniformization in p-Cyclic Extensions of Algebraic Surfaces over Ground Fields of Characteristic p, Mat. Annalen, 153 (1964), pp. 81-96.

[3] S. Abhyankar, Ramification Theoretic Methods in Algebraic Geometry, Annals of Math. Studies No. 43, Princeton University Press 1959.

[4] S. ABHYANKAR, Uniformization in a p-cylic extension of a two dimensional regular local domain of residue field characteristic $p$, Festschrift zur Gedächtnisfeier f'ur Karl Weierstrass, 18151965, Wissenschaftliche Abhandlungen des Landes Nordrhein-Westfalen, vol. 33, pp. 243317, K’oln und Opladen: Westdeutscher Verlag 1966. 
[5] S. Abhyankar, Nonsplitting of Valuations in Extensions of Two Dimensional Regular Local Domains, Math Annalen, 170 (1967), pp. 87-144.

[6] S. Abhyankar, An Algorithm on Polynomials in One Indeterminate with Coefficients in a Two Dimensional Regular Local Domain, Annali di Matematica pura ed applicata, 71 (1966), pp. 25-60.

[7] S. Abhyankar, Resolution of Singularities of Embedded Algebraic Surfaces, Academic Press, 1966.

[8] S. ABhyankar, Good points of a hypersurface, Advances in Math., 68 (1988), pp. 87-256.

[9] D. Abramovich And A. J. De Jong, Smoothness, semistability and toroidal geometry, Journal of Algebraic Geometry, 6 (1997), pp. 789-801.

[10] A. Benito and O. Villamayor, Monoidal transformations of singularities in positive characteristic, preprint, arXiv:0811.4148.

[11] E. Bierstone and P. Milman, Canonical desingularization in characteristic zero by blowing up the maximal strata of a local invariant, Inv. Math., 128 (1997), pp. 207-302.

[12] A. Bravo, S. Encinas, and O. Villamayor, A simplified proof of desingularization and applications, Revista Matematica Iberoamericana, 21 (2005), pp. 349-458.

[13] A. Bravo And O. Villamayor, Singularities in positive characteristic, stratification and simplification of the singular locus, preprint arXiv:0807.4308.

[14] V. Cossart and O. Piltant, Resolution of singularities of threefolds in positive characteristic I, J. Algebra, 320 (2008), pp. 1051-1082.

[15] V. Cossart and O. Piltant, Resolution of singularities of threefolds in positive characteristic II, J. Algebra, 321 (2009), pp. 1836-1976.

[16] V. Cossart, U. Jannsen, And S. Saito, Canonical embedded and non embedded resolution of singularities for excellent two-dimnensional schemes, preprint, arXiv:0905.2191.

[17] S. D. Cutкosky, Resolution of Singularities, American Mathematical Society, 2004.

[18] S. D. Cutkosky, Resolution of singularities for 3-folds in positive characteristic, Amer. J. Math., 131 (2009), pp. 59-127.

[19] A. J. DE Jong, Smoothness, semistability and alterations, Publ. Math. I.H.E.S., 83 (1996), pp. 51-93.

[20] S. EnCINAS And H. Hauser, Strong resolution of singularities in characteristic zero, Comment. Math. Helv., 77 (2002), pp. 821-845.

[21] S. Encinas And O. Villamayor, A new proof of desingularization over fields of characteristic zero, Proceedings of the international conference on Algebraic Geometry and singularities, Rev. Math. Iberoamericana, 19 (2003), pp. 339-353.

[22] A. Grothendieck and J. Dieudonné, Élements de Gémétrie Algébrique IV, Publ. Math. IHES, 28 (1966).

[23] A. Grothendieck And J. Murre, The tame fundamental group of a formal neighborhood of a divisor with normal crossings on a scheme, LNM 208, Springer Verlag, 1977.

[24] H. HAuser, Excellent surfaces and their taut resolution, Resolution of singularities, (Obergurgl, 1997), Progr. Math 181, Birkhäuser, Basel, 2000, pp. 341-373.

[25] H. HAUsER, Wild singularities and kangaroo points for the resolution of singularities in positive characteristic, preprint.

[26] H. Hironaka, Resolution of singularities of an algebraic variety over a field of characteristic zero, Annals of Math., 79 (1964), pp. 109-326.

[27] H. Hironaka, Desingularization of excellent surfaces, Advanced Study Seminar in Algebraic Geometry, Bowdoin College, 1967, Notes by B.M. Bennet, in Appendix to Resolution of surface singularities, V. Cossart, J. Giraud, and U. Orbanz, LNM 1101, Springer Verlag, Heidelberg, Berlin, New York, 1984.

[28] H. Hironaka, Characteristic polyhedra of singularities, J. Math. Kyoto Univ., 7 (1967), pp. 251-293.

[29] H. HiRonaKA, Idealistic exponents of singularity, Algebraic geometry, pp. 52-125, J.J. Sylvester Sympos. 1976, Johns Hopkins Univ. Press, Baltimore, 1977.

[30] H. HironakA, Theory of infinitely near singular points, J. Korean Math. Soc., 40 (2003), pp. 901-920.

[31] H. Hironaka, A program for resolution of singularities, in all characteristics $p>0$ and in all dimensions, Lecture notes from the school and conference on Resolution of singularities, Trieste, 2006.

[32] H. KAWANOUE, Toward resolution of singularities over a field of positive characteristic (The Kawanoue program), Part I. , Foundation of the program: the language of the idealistic filtration. AG/0607009.

[33] H. Kawanoue and K. Matsuki, Toward resolution of singularities over a field of positive characteristic (The Kawanoue program), Part II, Basic invariants associated to the idealistic 
filtration and their properties. AG/0612008.

[34] J. Kollár, Lectures on resolution of singularities, Annals of Math. Studies 166. Princeton Univ. Press, Princeton, 2007.

[35] H. Knaf And F. V. Kuhlmann, Every place admits local uniformization in a finite extension of the function field, Adv. Math., 221 (2009), pp. 428-453.

[36] W. KRull, Allgemeine Bewertungstheorie, Journal f'ur die reine und angewandte Mathematik, 167 (1931), pp. 160-196.

[37] J. Lipman, Desingularization of 2-dimensional schemes, Annals of Math., 107 (1978), pp. 115207.

[38] H. Matsumura, Commutative Algebra, second edition, Benjamin/Cummings (1969).

[39] T. T. MoH, On a Newton polygon approach to the uniformization of singularities in characteristic $p$, in Algebraic geometry and singularities (La Rábida, 1991) pp. 49-93, Progr. Math. 134, Birkhäuser, Basel, 1996.

[40] U. Orbanz, Embedded Resolution of Algebraic Surfaces after Abhyankar (characteristic 0), in "Resolution of surface singularities", V. Cossart, J. Giraud, and U. Orbanz, LNM 1101, Springer Verlag, Heidelberg, Berlin, New York, 1984.

[41] M. Spivakovsky, A counterexample to the theorem of Beppo Levi in three dimensions, Invent. Math., 96 (1989), pp. 181-183.

[42] B. TeIssien, Valuations, deformations and toric geometry, Valuation theory and its applications II, F. V. Kuhlmann, S. Kuhlmann and M. Marshall, editors, Fields Institute Communications 33, Amer. Math. Soc., Providence, RI, pp. 361-459.

[43] M. TEMKIN, Inseparable local uniformization, preprint, arXiv:0804.1554.

[44] O. Villamayor, Constructiveness of Hironaka's resolution, Ann. Scient. Ecole Norm. Sup., 22 (1989), pp. 1-32.

[45] J. Wlodarczyk, Simple Hironaka resolution in characteristic zero, J. Amer. Math. Soc., 18 (2005), pp. 779-822.

[46] O. ZARISKI, Reduction of singularities of algebraic three-dimensional varieties, Ann. of Math., 45 (1944), pp. 472-542.

[47] O. Zariski and P. Samuel, Commutative Algebra Vol. I and Vol. II, Van Nostrand, Princeton, 1960. 